Portland State University

PDXScholar

Dissertations and Theses

Dissertations and Theses

Summer 8-30-2018

\title{
Using Strong Laser Fields to Produce Antihydrogen lons
}

Christopher Michael Keating

Portland State University

Follow this and additional works at: https://pdxscholar.library.pdx.edu/open_access_etds

Part of the Physics Commons

Let us know how access to this document benefits you.

Recommended Citation

Keating, Christopher Michael, "Using Strong Laser Fields to Produce Antihydrogen lons" (2018).

Dissertations and Theses. Paper 4519.

https://doi.org/10.15760/etd.6403

This Dissertation is brought to you for free and open access. It has been accepted for inclusion in Dissertations and Theses by an authorized administrator of PDXScholar. Please contact us if we can make this document more accessible: pdxscholar@pdx.edu. 
Using Strong Laser Fields
to Produce Antihydrogen Ions

to Produce Antihydrogen lons

by

Christopher M. Keating

A dissertation submitted in partial fulfillment of the requirements for the degree of
Doctor of Philosophy
in
Applied Physics
Dissertation Committee:
Jack C. Straton, Chair
Pui (Peter) Leung
Reuben Simoyi
Theresa McCormick
Rolf Koenenkamp
Portland State University
2018


(c) 2018 Christopher M. Keating 


\begin{abstract}
We provide estimates of both cross section and rate for the stimulated attachment of a second positron into the $\left(1 s^{2}{ }^{1} S^{e}\right)$ state of the $\bar{H}^{+}$ion using Ohmura and Ohmura's (1960 Phys. Rev. 118 154) effective range theory, Reiss's strong field approximation (1980 Phys. Rev. A 22, 1786), and the principle of detailed balancing. Our motivation for producing $\bar{H}^{+}$ion include its potential to be used as an intermediate state in bringing antihydrogen to ultra-cold (sub-mK) temperatures required for a variety of studies, which include both spectroscopy and the probing of the gravitational interaction of the anti-atom. We show that both cross section and rate are increased with the use of a resonant laser field.
\end{abstract}




\section{Acknowledgements}

I would like to express my very great appreciation to Jack C. Straton, my research advisor, for his patient guidance, enthusiastic encouragement, and dedicated attention. I would also like to thank Peter Leung for his amazing instruction and honest advice. I am grateful also to Reuben Simoyi, from whom I have had the great joy of learning about chaotic systems, as well as the immense importance of Banach spaces! My thanks also to Theresa McCormick for introducing me to the complex physics of molecules. I am grateful to Rolf Koenenkamp for taking the time to carefully critique my work, even though the weather was perfect for flying.

I am also indebted to Mike Charlton. It was by his suggestions that the idea that we might be able to use strong laser fields to produce antihydrogen ions came to be.

Finally, I wish to thank Inggrid Chandranata for her love and emotional support.

Dedicated to my Family. 


\section{Table of Contents}

Abstract $\quad$ i

Acknowledgements $\quad$ ii

List of Figures $\quad$ vi

$\begin{array}{lll}\text { Chapter } 1 & \text { Motivation } & 1\end{array}$

1.1 CP Violation . . . . . . . . . . . . . . . . . 1

1.2 Tests of Symmetry . . . . . . . . . . . . . . . . 3

$\begin{array}{lll}\text { Chapter } 2 & \text { Basics of Laser-Atom Interactions } & 6\end{array}$

2.1 Introduction . . . . . . . . . . . . . . . 6

2.2 Classical Description of a Laser Field . . . . . . . . . . . . . . . . . . 11

2.3 Time-Dependent Schrödinger Equation . . . . . . . . . . . . . 13

2.4 Interaction of an Atomic System with an EM Field . . . . . . . . . . 15

2.5 Dipole Approximation . . . . . . . . . . . . . . . . . . . 17

2.6 Gauge Transformations . . . . . . . . . . . . . . . . . . . . . . . 19

2.7 Pulsed Laser Fields . . . . . . . . . . . . . . . . . . . . . . . . . . . . 20

2.8 Two Common Dipole Gauges . . . . . . . . . . . . . . . . . . . . . . 24

2.8.1 The Velocity Gauge . . . . . . . . . . . . . . 26

2.8.2 The Length Gauge . . . . . . . . . . . . . . . . . . . . . . . 27 
2.9 Interaction of a Single Electron with an EM Field . . . . . . . . . . 30

Chapter $3 \quad$ Rates, Cross-Sections, and Detailed Balancing 33

3.1 Rate Equation . . . . . . . . . . . . . . . . . . 33

3.2 Detailed Balancing . . . . . . . . . . . . . . . 36

$\begin{array}{lll}\text { Chapter } 4 & \text { Perturbation Theory } & 38\end{array}$

4.1 First Order Perturbation . . . . . . . . . . . . . . . . 38

4.2 Periodic Perturbation . . . . . . . . . . . . . . . 41

4.3 Application to Two Particle Systems . . . . . . . . . . . . . . . 45

$\begin{array}{lll}\text { Chapter } 5 & \text { The Negative Hydrogen Ion } & 47\end{array}$

5.1 Photodetachment $(\mathrm{PD})$. . . . . . . . . . . . 47

5.2 Spontaneous Radiative Attachment (RA) . . . . . . . . . . 55

5.3 Stimulated Attachment $(\mathrm{SA}) \ldots \ldots \ldots$. . . . . . . . 65

$\begin{array}{lll}\text { Chapter } 6 & \text { Beyond Perturbation } & 70\end{array}$

6.1 S-matrix and T-matrix . . . . . . . . . . . . 70

6.2 Strong Field Approximation (SFA) . . . . . . . . . . . . 73

6.3 Applicaton of the Strong Field Approximation . . . . . . . . . . . 75

6.4 The Weak Field Limit . . . . . . . . . . . . . . . . . . . 84

6.5 The Function $\Xi_{n}\left(k \alpha_{0},-\frac{z}{2}\right) \ldots \ldots \ldots \ldots$. . . . . . . . 85

6.6 Strong Field Photodetachment (PD) . . . . . . . . . . . . 86

6.7 Strong Field Spontaneous Radiative Attachment (RA) . . . . . . . 88

6.8 Strong Field Stimulated Attachment (SA) . . . . . . . . . . . . . 89

$\begin{array}{lll}\text { Chapter } 7 & \text { Conclusion } & 95\end{array}$

7.1 Concluding Remarks . . . . . . . . . . . . . . . . 95 
$\begin{array}{ll}\text { Bibliography } & 97\end{array}$

$\begin{array}{lll}\text { Appendix A } & \text { Ultra Short Pulses } & 104\end{array}$

Appendix B Perturbation with Pulse Shape Function 110

$\begin{array}{lll}\text { Appendix C } & \text { Effective Range Theory } & 113\end{array}$

Appendix D Derivation of the Function $\Xi_{n}\left(k \alpha_{0},-\frac{z}{2}\right) \quad 120$ 


\section{List of Figures}

2.1 Intensity vs. Frequency: The dotted line is the relativitic limit. The dashed line is the dipole limit. The dot-dashed line is the perturbation

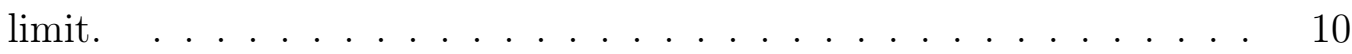

2.2 Electric Field vs. Time: Pulse period is $70 \mathrm{fs}$. Wavelength is $800 \mathrm{~nm}$. Notice, there are more than 10 optical periods within the pulse. . . .

2.3 Intensity vs. Time: Pulse period is $70 \mathrm{fs}$. Wavelength is $800 \mathrm{~nm}$. . . 25

2.4 Potential vs. Distance: In the length gauge, the interaction acts like a scalar potential that modifies the Coulomb potential. For this reason, many researchers like to use the length gauge. . . . . . . . . . .

5.1 Effective Range Potential: The potential felt by the second electron is approximated by a finite square well. The $\mathrm{x}$-axis is the distance in the

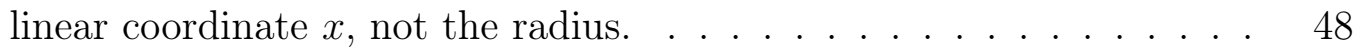

5.2 Cross Section vs. Momentum for Photodetachment (PD) . . . . . . 52

5.3 Cross Section vs. Electron Energy for Photodetachment (PD) . . . 53

5.4 Cross Section vs. Photon Energy for Photodetachment (PD) . . . . 54

5.5 Cross Section vs. Wavelength for Photodetachment (PD). For comparison and agreement with experiments see Keating [24, 25]. . . . . 55

5.6 Cross Sections for Spontaneous Radiative Attachment (RA): Left - vs. Momentum. Right - vs. Electron Energy. . . . . . . . . . . . . 58 
5.7 Maxwell-Boltzmann Distributions: Left - Electron. Right - Proton.

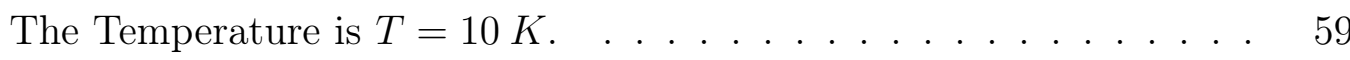

6.1 The quiver amplitude $\boldsymbol{\alpha}_{0}$, the shiver amplitude $\boldsymbol{\beta}_{0}$, and the direction of propagation k. . . . . . . . . . . . . . . . . 78

6.2 Cross Section vs. Wavelength for Photodetachment (PD). The dotted line is $z=0.5$. The dashed line is the dipole limit $z=0.01$. . . . .

6.3 Cross Section (per photon) vs. Wavelength for Stimulated Attachment (SA). The dotted line is $z=0.5$. The dashed line is the dipole limit $z=0.01 \ldots \ldots \ldots \ldots \ldots \ldots \ldots$ 


\section{Chapter 1}

\section{Motivation}

\subsection{CP Violation}

The beautiful relativistic equation of motion for the electron is called the Dirac Equation,

$$
i \hbar \gamma^{\mu} \partial_{\mu} \Psi=m c \Psi
$$

proposed by British physicist Paul Dirac in 1928. The electron wavefunction $\Psi$, describes everything there is to know about the electron (i.e. its position, momentum). Einstein's special relativity is contained within the equation, and Paul Dirac believed that equations expressing the natural world ought to be "Beautiful" and therefore his beautiful equation must be true. But there was a problem with Dirac's equation. The equation had twice the number of solutions one should expect. One solution described the electron, and the other solution appeared to describe something identical to the electron but which had the opposite electric charge. Some speculated that, perhaps, quantum theory just was not quite right, and the second solution had to be ignored. But to Pual Dirac, this equation was so beautful that it just had to be correct, which meant that the second solution had to be physical as well [1].

Four years later, Carl Anderson provided the experimental proof of Dirac's theory. Anderson was looking at cosmic rays coming from outer space. Anderson used a cloud chamber with a supersaturated vapor of water. When a charge particle interacts with 
the vapor, the fluid becomes ionized resulting in condensation. In this way, high energy ions will leave a trail on condensed liquid in the cloud chamber. Anderson built his cloud chamber and surrounded it with an electromagnet creating a uniform magnetic field, and photographed the vapor trail of the cosmic rays. He noticed that there appeared to be both positively and negatively charged particles by the two directions the particles curved in the magnetic field. By measuring the path's rate of curvature for the positively charged particles, he determined their mass to be the same as the electron. In order to know from which direction the particle was entering his cloud chamber, and thus determine the sign of the charge was in fact positive, Anderson placed a $6 \mathrm{~mm}$ lead plate across the center of the cloud chamber slowing the particle down so that its track curved more sharply. He called the positively charged particle the "positron." For discovering the first experimental evidence of antimatter, the positron, Anderson shared the 1936 Nobel prize in physics with Victor Hess [2].

According to the Dirac equation, there is symmetry between matter and antimatter. That is, they should behave identically. However at present, there appears to be more matter than antimatter in the Universe. One way to explain the lack of antimatter in the Universe is an asymmetry between matter and anitmatter. That is, there might be some difference in the behavoir of antimatter. How did we get from a Universe in the past with half antimatter to a Universe today with hardly any antimatter at all? This issue is called $\mathrm{CP}$ violation. The $\mathrm{C}$ stands for Charge conjugation $(q$ goes to $-q$ ) and the $\mathrm{P}$ stands for Parity transformation $(\mathbf{r}$ goes to $-\mathbf{r}$ ). In fact, both Charge conjugation and Parity transformation are maximally violated in the weak interaction. Dirac's equation predicts CP symmetry. However, CP symmetry cannot be true if the Universe changes from being half antimatter in the past to being much less than half at present. 


\subsection{Tests of Symmetry}

The focus of this study will be the production of the positive antihydrogen ion. However, the motivation for this study is centered on the tests of symmetry in nature. Any Lorentz invariant local quantum field theory with a Hermitian Hamiltonian must

obey the CPT theorem. Here the $\mathrm{T}$ stands for Time reversal $(t$ goes to $-t)$. Time reversal is likely to be weakly violated in the weak interaction. Since the Standard Model of Physics is a Lorentz invariant local quantum field theory with a Hermitian Hamiltonian, it must have physical laws that have symmetry under the simultaneous transformation of charge conjugation, parity transformation, and time reversal. CP violation was first observed by J. H. Christenson, J. W. Cronin, V. L. Fitch, R. Turlay in the $2 \pi$ decay of the $K_{2}^{0}$ meson in 1964 [3]. For their work, James Cronin and Val Fitch were awarded the 1980 Nobel Prize in Physics, for proving that certain subatomic reactions do not adhere to fundamental symmetry principles. The new age of experimental interest in $\mathrm{CP}$ violation provided direct evidence of $\mathrm{CP}$ violation. In 1999, Fermi Lab compared the decay rates of K mesons using a subset of data from the $\mathrm{KTeV}$ experiment, and definitively established the existence of $\mathrm{CP}$ violation [4]. That same year, CERN also announced the same direct $\mathrm{CP}$ violation for the K mesons using data taken in 1997 by the NA48 experiment [5]. Stanford's Linear Accelerator BABAR Collaboration and the Belle experiment at the High Energy Accelerator Research Organization in Japan measured time-dependent CP violations in neutral B decays [6, 7]. In 2013, the LHCb Collaboration used $p p$ collision data collected in 2011 and reported the direct CP violation in $B_{s}^{0} \rightarrow K^{-} \pi^{+}$decays [8]. In 2014, BABAR and Belle also reported results corresponding to violation of CP in B mesons.

Unlike the electroweak theory, there is as yet no experimental violation of $\mathrm{CP}$ 
symmetry known for the strong force. In the electroweak theory the gauge fields couple to chiral currents of fermionic fields; in quantum chromodynamics the gluons couple to vector currents.

$\mathrm{CP}$ violating processes that are known to date are, in fact, orders of magnitude smaller $\left(\sim 10^{-3}\right)$ than that needed to explain the excess of matter over antimattter. This leads us to look for more antimatter in the Universe, or look for new underlying physics. There is an excellent review of the current state of the issue of $\mathrm{CP}$ violations in Nature by John Ellis [9].

The main remaining experimental tests on the CPT theorem are: 1) the spectroscopic comparison of hydrogen and antihydrogen and 2) the test of gravity, or the weak equivalence principle for antimatter [10]. In order to conduct such experiments, the production and trapping of antihydrogen is vital. In 2002, ATHENA successfully produced low-energy antihydrogen by the controlled mixing of cold positrons in an antiproton cloud [11]. ATRAP produced cold antihydrogen by repeatedly colliding antiprotons with cold positrons in nested Penning traps [12]. These two experiments have produced on the order of 100,000 antihydrogen atoms. ALPHA has recently reported confinement times for antihydrogen of 1,000 seconds [13]. New experiments are currently underway to measure the spectra of antihydrogen $[14,15,16]$. The most recent results to measure the spectra have been consistent with CPT invariance [17].

The trapping and cooling of positrons and antiprotons is key to producing antihydrogen. A small fraction of the total yield can be held if efficient cooling techniques can produce sub-mK particles. Cooling of antiparticles has been done two ways: buffer gas accumulators [18] and Penning traps [19]. Particles cooled by buffer gas accumulators can be transferred to a high vacuum apparatus for antihydrogen formation [20]. Antiprotons are frequently cooled in Penning-type traps using clouds of electrons and, more recently, cryogenic temperatures have been reached using evapo- 
rative and adiabatic techniques [21, 22].

In the present work, we consider the radiative attachment of a positron to antihydrogen to form the antihydrogen positive ion, $\bar{H}^{+}$, via the reaction

$$
\bar{H}(1 s)+e^{+} \rightarrow \bar{H}^{+}\left(1 s^{2}{ }^{1} S^{e}\right)+\hbar \omega
$$

as well as the stimulated attachment of a positron to antihydrogen via the reaction,

$$
\bar{H}(1 s)+e^{+}+\mathcal{N} \hbar \omega \rightarrow \bar{H}^{+}\left(1 s^{2}{ }^{1} S^{e}\right)+(\mathcal{N}+1) \hbar \omega .
$$

Our interest in the production of $\bar{H}^{+}$lies in its potential to be used as an intermediate in bringing antihydrogen to ultra-cold (sub-mK) temperatures required for gravitational studies, first suggested by Walz and Hänsh [23]. Because of its net charge, sympathetic cooling with $\bar{H}^{+}$using laser-cooled positively charged ions of ordinary matter like $\mathrm{Be}^{+}$is more efficient than with a neutral atom such as $\bar{H}$. The biggest obstacle to implementing $\bar{H}^{+} \rightarrow \bar{H}$ cooling process is the efficiency with which the antihydrogen ion can be produced. In our previous works, we provided estimates of the cross section for radiative attachment for a second positron making the $\left(1 s^{2}{ }^{1} S^{e}\right)$ state of the $\bar{H}^{+}$ion using Ohmura and Ohmura's effective range theory, as well as with an explicitly correlated 200-term two-positron exponential wavefunction $[24,25]$. In that work, we obtained the rate coefficient for attaching a second positron to antihydrogen as a function of temperature. Estimates of the reaction rates were around $5 \times 10^{-3} \mathrm{~s}^{-1}$. Although the reaction rates were low, given the long storage time achieved by ALPHA, the formation of $\bar{H}^{+}$might just be observable. In order to increase the reaction rate, this work will use a resonant laser field that should stimulate the process of radiative attachment, thereby increasing the reaction rate. 


\section{Chapter 2}

\section{Basics of Laser-Atom Interactions}

\subsection{Introduction}

In recent years, short pulsed lasers have reached an intensity that allows the laser field to compete with the Coulomb force in an atomic system. If the radiation is sufficiently intense, then strong field effects will begin to play a significant role, and taking them into account will be vital to understanding the dynamics of the system.

Typically, at intensities (i.e. irradiance) of the order of $10^{8} \mathrm{~W} / \mathrm{cm}^{2}$ one will begin to observe strong field effects in laser-assisted electron-atom collisions, while at intensities of only $10^{10} \mathrm{~W} / \mathrm{cm}^{2}$ one will begin to observe multiphoton ionization in atoms. In fact, such intensities are now considered to be rather modest. Short pulsed lasers have become available, having intensities of the order of, or exceeding, the atomic unit of intensity

$$
I_{a}=\frac{1}{2} \epsilon_{0} c E_{a}^{2} \approx 3.5 \times 10^{16} \mathrm{~W} / \mathrm{cm}^{2}
$$

where $c=2.998 \times 10^{8} \mathrm{~m} / \mathrm{s}=137.0 \mathrm{au}$, is the velocity of light in vacuo, $\epsilon_{0}=$

$8.854 \times 10^{-12} \frac{C^{2}}{N \cdot m^{2}}=\frac{1}{4 \pi} a u$, is the permittivity of free space and $E_{a}$ is the atomic unit of electric field strength, namely

$$
E_{a}=\frac{e}{\left(4 \pi \epsilon_{0}\right) a_{0}^{2}} \approx 5.1 \times 10^{9} \mathrm{~V} / \mathrm{cm}
$$


where $e=1.602 \times 10^{-19} \mathrm{C}=1 \mathrm{au}$, is the absolute value of the electron charge and $a_{0}=5.292 \times 10^{-11} \mathrm{~m}=1 \mathrm{au}$, is the Bohr radius of atomic hydrogen. For example, a typical Ti-Sapphire laser will have the power on order of $1 W$ and can be operated with a beam diameter of about $1 \mu \mathrm{m}$. This commercially available laser has an intensity

$$
I=\frac{P}{A} \approx \frac{1 \mathrm{~W}}{\left(1 \times 10^{-6} \mathrm{~m}\right)^{2}}=10^{12} \frac{\mathrm{W}}{\mathrm{m}^{2}}=10^{8} \frac{\mathrm{W}}{\mathrm{cm}^{2}},
$$

already in the range of strong field effects.

The number of photons in such a laser field is very large. For example, with the photon density given by $\Phi_{\text {photon }}=I /(\hbar \omega)$, a laser field due to an Ti-Sapphire laser with a modest intensity $I=10^{10} \mathrm{~W} / \mathrm{cm}^{2}$ in a coherence volume of $\mathcal{V} \approx \lambda^{3}$ $(\lambda=2 \pi c / \omega=800 \mathrm{~nm})$ over one period, has a number of photons equal t0

$$
\mathcal{N}=\frac{I \mathcal{V}}{c \hbar \omega} \approx 6.9 \times 10^{5} \text { photons }
$$

For a large (average occupation) number of photons in a coherent state, the higher order corrections that occur in the full relativitic field theory merely cause very small oscillations about the classical laser field (i.e. electromagnetic field). Thus, a classical (as opposed to second quantized) description of the laser field is adequate. However, the electrons' field will be treated by a quantum wavefunction. This is sometimes called a semi-classical approach. We will therefore treat the atomic system using non-relativitic quantum theory. We neglect relativistic effects that only occur when an atom interacts with an ultra-strong laser field defined as [26]

$$
U_{p} \gtrsim m c^{2},
$$


where

$$
U_{p}=\frac{e^{2} E_{0}^{2}}{4 m \omega^{2}}=\frac{e^{2}}{2 \epsilon_{0} m c} \frac{I}{\omega^{2}},
$$

is the ponderomotive energy of the laser atom system and $m c^{2}$ is the invariant energy of the electron. The ponderomotive energy is defined as the cycle-averaged quiver energy of a "free" electron in a laser field (See Joachain p. 55 [27]). The intensities at which the field becomes ultra-strong are

$$
I \gtrsim I_{\text {rel }}(\omega)
$$

where

$$
I_{r e l}(\omega)=\frac{2 \epsilon_{0} m^{2} c^{3}}{e^{2}} \omega^{2}=\left(\frac{m c^{2}}{\hbar \omega}\right) \frac{1}{\alpha} \frac{1}{2 \pi} m \omega^{3}
$$

Thus, if $\lambda=800 \mathrm{~nm}$, corresponding to a Ti-Sapphire laser $\left(\omega=2.355 \times 10^{15} \mathrm{~s}^{-1}=\right.$ $0.05696 \mathrm{au})$, relativistic effects will begin with intensities

$$
I_{\text {rel }} \approx 8.6 \times 10^{18} \mathrm{~W} / \mathrm{cm}^{2}
$$

In fact, non-dipole effects begin to become important well before ultra-strong (i.e. relativistic) effects. The condition at which non-dipole effects start to become important is when the quadrupole term becomes the same order as the dipole term. This can be summarized by the condition [28]

$$
\frac{U_{p}}{\hbar \omega} \gtrsim \frac{1}{\alpha}
$$

where $\alpha=\frac{e^{2}}{4 \pi \epsilon_{0} \hbar c}=7.29735308 \times 10^{-3} \approx 1 / 137.0$ is the fine-structure constant. Thus, 
with a Ti-Sapphire laser the condition at which dipole effects are important is

$$
I \gtrsim I_{\text {dip }}(\omega)
$$

where

$$
I_{d i p}(\omega)=\frac{2 \epsilon_{0} m \hbar c}{e^{2}} \omega^{3}=\frac{1}{\alpha^{2}} \frac{1}{2 \pi} m \omega^{3},
$$

or

$$
I_{d i p} \approx 3.6 \times 10^{15} \mathrm{~W} / \mathrm{cm}^{2} .
$$

The most important limitation to our analysis would be the point at which perturbation theory is no longer available. The point at which even higher order pertubation theory breaks down is

$$
U_{p} \gtrsim \hbar \omega,
$$

giving

$$
I_{0}(\omega)=\frac{2 \epsilon_{0} m \hbar c}{e^{2}} \omega^{3}=\frac{1}{\alpha} \frac{1}{2 \pi} m \omega^{3} .
$$

Thus, with a $\lambda=800 \mathrm{~nm}$ Ti-Sapphire laser, strong-field effects will begin at intensities

$$
I_{0} \approx 2.6 \times 10^{13} \mathrm{~W} / \mathrm{cm}^{2} .
$$

While processes involving lasers with intensities $I \ll I_{0}$ can be studied by using perturbation theory, the effects of strong laser fields with intensities of the order of, or exceeding, this condition must be analyzed by using non-perturbative approaches, which is the aim of this study. It is convenient to define a non-perturbative parameter,

$$
z \equiv \frac{U_{p}}{\hbar \omega}=\frac{I}{I_{0}}
$$




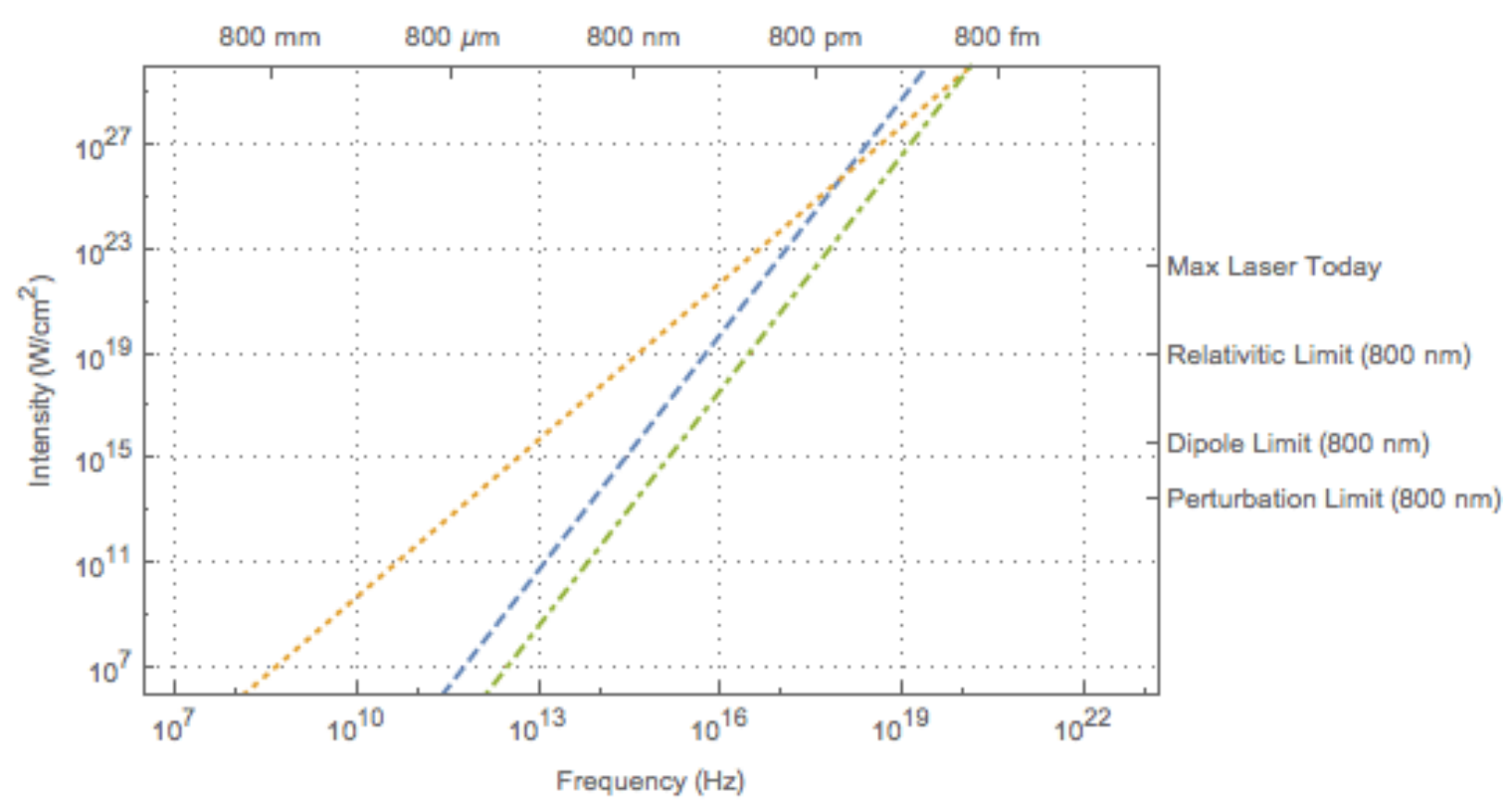

Figure 2.1: Intensity vs. Frequency: The dotted line is the relativitic limit. The dashed line is the dipole limit. The dot-dashed line is the perturbation limit.

where $z \gtrsim 1$ is where perturbation breaks down. We note that,

$$
U_{p}=\hbar \omega z=(1.5 \mathrm{eV}) \frac{I}{I_{0}} .
$$

The condition needed in order for single photon detachment is

$$
\hbar \omega \geq E_{B}+U_{p},
$$

where $E_{B}$ is the bound state energy. If the condition is not satisified (perhaps the frequency is very low), there can still be detachment. However, the detachment will require multiple photons such that

$$
n_{0} \hbar \omega \geq E_{B}+U_{p},
$$

where $n_{0}$ is the smallest integer that satitifies the inequality. This is called multiphoton 
detachment (ionization). In the case where the photon(s) energy is greater than the both binding energy and ponderomotive energy, the excess energy is given to the ejected electron's kinectic energy. We can define a multiphoton intensity parameter,

$$
z_{\text {multi }} \equiv \frac{E_{B}+U_{p}}{\hbar \omega}=\frac{E_{B}}{\hbar \omega}+z
$$

where $z_{\text {multi }} \geq 1$ is when single photon interactions are no longer possible. We point out that if the frequency (i.e. photon energy) is low in comparison to the bound state energy, multiphoton interactions can be required even at very small laser field intensities! Lastly, it is also possible to absorb more photons than the minimum needed to photodetach the electron. This is referred to as above-threshold ionization.

\subsection{Classical Description of a Laser Field}

We will begin with a quick review of the necessary classical theory of electromagnetic fields. The electromagnetic field of the laser is described by the electric and magnetic vector fields, $\mathbf{E}(\mathbf{r}, t)$ and $\mathbf{B}(\mathbf{r}, t)$, which satisfy the Maxwell's equations,

$$
\begin{gathered}
\boldsymbol{\nabla} \cdot \mathbf{E}=\frac{\rho}{\epsilon_{0}} \\
\nabla \cdot \mathbf{B}=0 \\
\nabla \times \mathbf{E}=-\frac{\partial \mathbf{B}}{\partial t} \\
\boldsymbol{\nabla} \times \mathbf{B}=\mu_{0} \mathbf{J}+\frac{1}{c^{2}} \frac{\partial \mathbf{E}}{\partial t},
\end{gathered}
$$

where $c=1 / \sqrt{\epsilon_{0} \mu_{0}}$ is the speed of light in vacuum. The electric and magnetic fields can be generated from scalar and vector potentials, $V(\mathbf{r}, t)$ and $\mathbf{A}(\mathbf{r}, t)$, respectively, by the following relations:

$$
\mathbf{E}=-\nabla V-\frac{\partial \mathbf{A}}{\partial t}
$$


and

$$
\mathbf{B}=\nabla \times \mathbf{A} .
$$

The Maxwell's equations written in terms of the scalar and vector potentials, $V(\mathbf{r}, t)$ and $\mathbf{A}(\mathbf{r}, t)$, are

$$
\begin{gathered}
\nabla^{2} V+\frac{\partial}{\partial t}(\boldsymbol{\nabla} \cdot \mathbf{A})=-\frac{\rho}{\epsilon_{0}} \\
\nabla^{2} \mathbf{A}-\frac{1}{c^{2}} \frac{\partial^{2} \mathbf{A}}{\partial t^{2}}-\boldsymbol{\nabla}\left(\boldsymbol{\nabla} \cdot \mathbf{A}+\frac{1}{c^{2}} \frac{\partial V}{\partial t}\right)=-\mu_{0} \mathbf{J} .
\end{gathered}
$$

The Poynting vector is defined as

$$
\mathbf{S}=\frac{1}{\mu_{0}}(\mathbf{E} \times \mathbf{B}),
$$

which is the rate of energy flow through a unit area normal to the direction of propagation. Both the intensity (irradiance) and energy flux are defined through the Poynting vector. Intensity is defined as the time averaged Poynting vector. It is,

$$
\begin{gathered}
I=|\langle\mathbf{S}\rangle| \\
=\frac{1}{2} \epsilon_{0} c E_{0}^{2}=\frac{1}{2} \epsilon_{0} c \omega^{2} A_{0}^{2}=\frac{\mathcal{N} \hbar \omega c}{\mathcal{V}}
\end{gathered}
$$

where the first equality is definition and the latter are for sinusoidal fields, while $\mathcal{N}$ is the occupation number (number of photons) and $\mathcal{V}$ is volume. Energy (radiant) flux is the rate of energy flow through a specified surface. Energy flux is defined by

$$
\Phi=\int \mathbf{S} \cdot d \mathbf{a},
$$

where $d \mathbf{a}$ is the surface element. However, the time-average of the Poynting vector is commonly used because it is the quantity that radiation detectors are able to measure. 
It is,

$$
\Phi=\int\langle\mathbf{S}\rangle \cdot d \mathbf{a}
$$

The photon flux is defined as the rate of photons per unit area,

$$
\Phi_{\text {photon }}=\frac{\Phi}{\hbar \omega}
$$

The energy density is defined as

$$
u=\frac{1}{2} \epsilon_{0} E^{2}+\frac{1}{2 \mu_{0}} B^{2}
$$

\subsection{Time-Dependent Schrödinger Equation}

The non-relativistic reduced Hamiltonian of an electron in an electromagnetic field is described, in an arbitrary gauge, by the scalar potential $V(\mathbf{r}, t)$ and the vector potential $\mathbf{A}(\mathbf{r}, t)$ as

$$
\begin{array}{rlc}
H(t) & = & \frac{1}{2 m}(\mathbf{p}+e \mathbf{A})^{2}-e V \\
& =\frac{\mathbf{p}^{2}}{2 m}+\frac{e}{2 m}(\mathbf{A} \cdot \mathbf{p}+\mathbf{p} \cdot \mathbf{A})+\frac{e^{2}}{2 m} \mathbf{A}^{2}-e V,
\end{array}
$$

where small spin-dependent terms have been neglected and the vector potential $\mathbf{A}(\mathbf{r}, t)$ has the units of [potential] · [time]/[length] (i.e Volts/c in SI units). Here $m$ is the reduced mass of the electron $\left(m=m_{e} m_{p} /\left(m_{e}+m_{p}\right)=9.104425 \times 10^{-31} \mathrm{~kg}=\right.$ $0.9994 m_{e}$. However, this is close enough to the electron's mass for our purposes and we will use $m \approx m_{e}=9.109 \times 10^{-31} \mathrm{~kg}$ ), and we will neglect the small mass polarization terms that are of the same order as the relativistic corrections. In the above equations, $\mathbf{p}$ is the canonical momentum operator of the electron. Working in the Schrödinger picture and using the position representation in which $\mathbf{p}=-i \hbar \nabla$, 
the corresponding Time-Dependent Schrödinger Equation (TDSE) is as follows

$$
i \hbar \frac{\partial}{\partial t} \Psi(\mathbf{r}, t)=\left[-\frac{\hbar^{2}}{2 m} \nabla^{2}-e V-i \hbar \frac{e}{2 m} \mathbf{A} \cdot \boldsymbol{\nabla}-i \hbar \frac{e}{2 m} \boldsymbol{\nabla} \cdot \mathbf{A}+\frac{e^{2}}{2 m} \mathbf{A}^{2}\right] \Psi(\mathbf{r}, t),
$$

Note that we can impose an electromagnetic gauge freedom, namely the Coulomb (radiation) gauge, $\boldsymbol{\nabla} \cdot \mathbf{A}^{C}=0$,

$$
\begin{array}{rll}
\boldsymbol{\nabla} \cdot\left(\mathbf{A}^{C} \Psi^{C}\right) & =\mathbf{A}^{C} \cdot\left(\boldsymbol{\nabla} \Psi^{C}\right)+\left(\boldsymbol{\nabla} \cdot \mathbf{A}^{C}\right) \Psi^{C} \\
& = & \mathbf{A}^{C} \cdot\left(\boldsymbol{\nabla} \Psi^{C}\right) .
\end{array}
$$

Alternatively, the same relation can be imposed by the more restrictive dipole approximation. In that case the TDSE is simply

$$
i \hbar \frac{\partial}{\partial t} \Psi(\mathbf{r}, t)=\left[-\frac{\hbar^{2}}{2 m} \nabla^{2}-e V-i \hbar \frac{e}{m} \mathbf{A} \cdot \boldsymbol{\nabla}+\frac{e^{2}}{2 m} \mathbf{A}^{2}\right] \Psi(\mathbf{r}, t),
$$

where the superscripts denoting the Coulomb gauge are dropped. When the Coulomb gauge is used, then it is the vector potential that includes all transverse fields (i.e. propagating laser fields, radio waves, etc.). The word transverse has the meaning that such a field has an electric field in one direction, a magnetic field perpendicular to it, and a direction of propagation perpendicular to both of those fields. It is for this reason that the Coulomb gauge is sometimes referred to as the transverse gauge. We will see that within the dipole approximation, the length gauge can be utilized, and a transverse field (i.e. propagating plane-wave field) can in some sense be described by a scalar potential, although it is best to not take this mental picture too seriously. However, longitudinal fields (i.e. Coulomb potential) can be described by a scalar potential, and are conveniently (and appropriately!) described by such scalar potentials. 


\subsection{Interaction of an Atomic System with an EM Field}

We now consider the interaction of an electromagnetic field with an atomic system composed of a nucleus of atomic number $Z$ and $N$ atomic electrons. We will neglect relativistic effects which only occur when an atom interacts with an ultra-strong laser field. We neglect small effects due to the interaction between the nucleus and the electromagnetic field. In the same spirit, we drop small mass polarization terms and take the nucleus to be the origin of the coordinates.

Let us first consider the case $N=1$, corresponding to hydrogenic atoms and ions. We must then include in the Hamiltonian the electrostatic Coulomb potential $V=-Z e /\left(4 \pi \epsilon_{0} r\right)$ between the electron and the nucleus. It is convenient to regard this electrostatic interaction as an additional potential energy term, while the radiation field is described in the Coulomb gauge in terms of a vector potenial $\mathbf{A}(\mathbf{r}, t)$ alone, as discussed above. The TDSE reads

$$
i \hbar \frac{\partial}{\partial t} \Psi(\mathbf{r}, t)=\left[-\frac{\hbar^{2}}{2 m} \nabla^{2}-\frac{Z e^{2}}{\left(4 \pi \epsilon_{0}\right) r}-i \hbar \frac{e}{m} \mathbf{A} \cdot \boldsymbol{\nabla}+\frac{e^{2}}{2 m} \mathbf{A}^{2}\right] \Psi(\mathbf{r}, t) .
$$

We can rewrite this equation in the form

$$
i \hbar \frac{\partial}{\partial t} \Psi(\mathbf{r}, t)=\left[H_{0}+H_{i n t}(t)\right] \Psi(\mathbf{r}, t)
$$

where

$$
H_{0}=-\frac{\hbar^{2}}{2 m} \nabla^{2}-\frac{Z e^{2}}{\left(4 \pi \epsilon_{0}\right) r}
$$

is the time-independent hydrogenic Hamiltonian describing the hydrogenic atom (ion) 
in the absence of the radiation field, and

$$
\begin{aligned}
H_{i n t}(t) & =-i \hbar \frac{e}{m} \mathbf{A} \cdot \boldsymbol{\nabla}+\frac{e^{2}}{2 m} \mathbf{A}^{2} \\
& =\frac{e}{m} \mathbf{A} \cdot \mathbf{p}+\frac{e^{2}}{2 m} \mathbf{A}^{2}
\end{aligned}
$$

is the interaction Hamiltonian describing the interaction of the hydrogenic atom with the radiation field. In order to generalize these results to the case of N-electron atom (ion), we have the field-free Hamiltonian

$$
H_{0}=\frac{1}{2 m} \sum_{i=1}^{N} \mathbf{p}_{i}^{2}+V=-\frac{\hbar^{2}}{2 m} \sum_{i=1}^{N} \nabla_{i}^{2}+V,
$$

where $V$ is the sum of all the interactions within the atomic system in the absence of the radiation field. For example, if all but Coulomb interactions are neglected, we have

$$
V=-\sum_{i=1}^{N} \frac{Z e^{2}}{\left(4 \pi \epsilon_{0}\right) r_{i}}+\sum_{i<j=1}^{N} \frac{e^{2}}{\left(4 \pi \epsilon_{0}\right) r_{i j}}
$$

where $r_{i j}=\left|\mathbf{r}_{i}-\mathbf{r}_{j}\right|$. The (Coulomb gauge) interaction Hamiltonian $H_{\text {int }}(t)$ for the N-electron atom (ion) is generalized to

$$
H_{\text {int }}(t)=\frac{e}{m} \sum_{i=1}^{N} \mathbf{A}\left(\mathbf{r}_{i}, t\right) \cdot \mathbf{p}_{i}+\frac{e^{2}}{2 m} \sum_{i=1}^{N} \mathbf{A}^{2}\left(\mathbf{r}_{i}, t\right)
$$

The explicit form of the TDSE is therefore as follows:

$$
\begin{gathered}
i \hbar \frac{\partial}{\partial t} \Psi\left(\mathbf{r}_{1}, \mathbf{r}_{2}, \ldots, \mathbf{r}_{N}, t\right) \\
=\left[\frac{1}{2 m} \sum_{i=1}^{N} \mathbf{p}_{i}^{2}+V+\frac{e}{m} \sum_{i=1}^{N} \mathbf{A}\left(\mathbf{r}_{i}, t\right) \cdot \mathbf{p}_{i}+\frac{e^{2}}{2 m} \sum_{i=1}^{N} \mathbf{A}^{2}\left(\mathbf{r}_{i}, t\right)\right] \Psi\left(\mathbf{r}_{1}, \mathbf{r}_{2}, \ldots, \mathbf{r}_{N}, t\right) .
\end{gathered}
$$




\subsection{Dipole Approximation}

Now we will assume that the wavelength $\lambda$ of the laser is large compared with the size of the atomic system (i.e. $\lambda \gg a_{0}$ ) under consideration and that the laser field intensity is not too high. When these two conditions are fulfilled, the dipole approxition can be made, which consists of neglecting the spatial variation of the radiation field across the atom. In this approximation, for an atom whose nucleus is located at the position $\mathbf{r}_{0}$, the vector potential $\mathbf{A}\left(\mathbf{r}_{0}, t\right)=\mathbf{A}(t)$ is spatially homogeneous (i.e. it depends only on the time variable) so that the Coulomb gauge condition is automatically satisfied. For a periodic vector potential the dipole approximation can be generated explicitly by

$$
\begin{array}{rlrl}
\mathbf{A}(\mathbf{r}, t) & = & A_{0} \cos \left(\mathbf{k}_{\omega} \cdot \mathbf{r}-\omega t\right) \hat{\boldsymbol{\epsilon}} \\
& =A_{0} \frac{1}{2}\left(e^{i\left(\mathbf{k}_{\omega} \cdot \mathbf{r}-\omega t\right)}+e^{-i\left(\mathbf{k}_{\omega} \cdot \mathbf{r}-\omega t\right)}\right) \hat{\boldsymbol{\epsilon}} \\
& = & A_{0} \frac{1}{2}\left(e^{i \mathbf{k}_{\omega} \cdot \mathbf{r}} e^{-i \omega t}+e^{-i \mathbf{k}_{\omega} \cdot \mathbf{r}} e^{i \omega t}\right) \hat{\boldsymbol{\epsilon}} \\
& \approx & A_{0} \frac{1}{2}\left(e^{-i \omega t}+e^{i \omega t}\right) \hat{\boldsymbol{\epsilon}} \\
& \approx & A_{0} \cos (\omega t) \hat{\boldsymbol{\epsilon}},
\end{array}
$$

where $\left|\mathbf{k}_{\omega}\right|=\omega / c$, and $e^{ \pm i \mathbf{k}_{\omega} \cdot \mathbf{r}} \approx 1$ is the dipole approximation. Another way to justify the dipole approximation is by considering the period of the laser field to be much longer than the time it takes for light to transverse the length of the atomic system. Of all the approximations we have made up until this point for strong fields, the dipole approximation would be the first to break down at sufficiently high intensities and low frequencies (yes, low frequencies!). For very strong fields, non-dipole effects must be taken into account and eventually, for ultra-strong fields, relativistic effects must be included. In this study, we will be working with wavelengths of order $\lambda \sim 800 \mathrm{~nm}$ and atoms of order $r \sim a_{0}=0.053 \mathrm{~nm}$. One might think that dipole approximation 
would break down at high frequencies because the wavelength will become on the order of the atomic system. While this is true at hard X-ray wavelengths close to $\lambda \sim 0.053 \mathrm{~nm}$, we are not interested in these wavelengths. Rather, we are more concerned with the low frequency breakdown when intensities are sufficently high. This breakdown has its origins in the conditions at which non-dipole effects start to become important, that is when the quadrupole term becomes the same order as the dipole term. The quadrupole term can be found in the $i \mathbf{k}_{\omega} \cdot \mathbf{r}$ term of the powers series expansion of the exponential,

$$
e^{i \mathbf{k}_{\omega} \cdot \mathbf{r}} \approx 1+i \mathbf{k}_{\omega} \cdot \mathbf{r}+\frac{1}{2 !}\left(i \mathbf{k}_{\omega} \cdot \mathbf{r}\right)^{2}+\ldots
$$

or one can expand in a series of spherical harmomics like

$$
e^{i \mathbf{k}_{\omega} \cdot \mathbf{r}}=4 \pi \sum_{l=0}^{\infty} \sum_{m=-l}^{l} i^{l} j_{l}\left(k_{\omega} r\right) Y_{l m}^{*}(\theta, \phi) Y_{l m}\left(\theta_{k}, \phi_{k}\right)
$$

We will not go beyond the dipole approximation in this work.

Returning our attention to the interaction Hamiltonian, we see that in the dipole approximation it takes the simplified form

$$
H_{\text {int }}(t)=\frac{e}{m} \mathbf{A}(t) \cdot \mathbf{P}+\frac{e^{2} N}{2 m} \mathbf{A}^{2}(t),
$$

where

$$
\mathbf{P}=\sum_{i=1}^{N} \mathbf{p}_{i}
$$

is the total momentum operator. The TDSE for an N-electron atom (ion) in an 
electromagnetic field can therefore be written in the dipole approximation as

$$
i \hbar \frac{\partial}{\partial t} \Psi\left(\mathbf{r}_{1}, \mathbf{r}_{2}, \ldots, \mathbf{r}_{N}, t\right)=\left[H_{0}+\frac{e}{m} \mathbf{A}(t) \cdot \mathbf{P}+\frac{e^{2} N}{2 m} \mathbf{A}^{2}(t)\right] \Psi\left(\mathbf{r}_{1}, \mathbf{r}_{2}, \ldots, \mathbf{r}_{N}, t\right)
$$

where $H_{0}$ is the field-free Hamiltonian.

\subsection{Gauge Transformations}

The phrase gauge transformation has taken on two meanings in the literature and so causes some confusion $[29,30]$. The first concept of gauge freedom is what we will call the electromagnetic gauge freedom, namely

$$
\begin{aligned}
\mathbf{A}^{\prime}(\mathbf{r}, t) & =\mathbf{A}(\mathbf{r}, t)+\nabla \Lambda(\mathbf{r}, t) \\
V^{\prime}(\mathbf{r}, t) & =V(\mathbf{r}, t)-\frac{\partial}{\partial t} \Lambda(\mathbf{r}, t) \\
\Psi^{\prime}(\mathbf{r}, t) & =\exp \left[-i \frac{e}{\hbar} \Lambda(\mathbf{r}, t)\right] \Psi(\mathbf{r}, t)
\end{aligned}
$$

where $\Lambda(\mathbf{r}, t)$ is any function that depends on position and time. With the electromagnetic gauge transformation, Maxwell's equations, along with the Schrödinger equation, remain satisfied

$$
\begin{aligned}
i \hbar \frac{\partial}{\partial t} \Psi^{\prime}(\mathbf{r}, t) & =\left[\frac{1}{2 m}\left(\mathbf{p}+e \mathbf{A}^{\prime}\right)^{2}-e V^{\prime}\right] \Psi^{\prime}(\mathbf{r}, t) \\
& =\left[\frac{\mathbf{p}^{2}}{2 m}+\frac{e}{2 m}\left(\mathbf{A}^{\prime} \cdot \mathbf{p}+\mathbf{p} \cdot \mathbf{A}^{\prime}\right)+\frac{e^{2}}{2 m} \mathbf{A}^{\prime 2}-e V^{\prime}\right] \Psi^{\prime}(\mathbf{r}, t)
\end{aligned}
$$

A strict gauge transformation will leave the Hamiltonian unchanged by the simultaneous transformation of $V, \mathbf{A}$ and $\Psi$. In this study, we have chosen the Coulomb (Radiation) gauge for the electromagnetic gauge freedom. Namely, we have chosen $\Lambda$ such that

$$
\nabla \cdot \mathbf{A}^{C}=0
$$


This amounts to a phase of [31]

$$
\begin{gathered}
\Lambda=\iint_{t}^{t-\left|\mathbf{r}-\mathbf{r}^{\prime}\right| / c} \frac{1}{\left|\mathbf{r}-\mathbf{r}^{\prime}\right|} \rho\left(\mathbf{r}^{\prime}, t^{\prime}\right) d^{3} x^{\prime} d t^{\prime} \\
=-\epsilon_{0} \iint_{t}^{t-\left|\mathbf{r}-\mathbf{r}^{\prime}\right| / c} \frac{1}{\left|\mathbf{r}-\mathbf{r}^{\prime}\right|}\left(\nabla^{2} V+\frac{\partial}{\partial t}(\boldsymbol{\nabla} \cdot \mathbf{A})\right) d^{3} x^{\prime} d t^{\prime} .
\end{gathered}
$$

The Coulomb gauge is now the conventional gauge choice in atomic physics. This standardization is likely due to the ease at which second quantization can be imposed on the vector potential, leading to quantum electrodynamics. Since the scalar potential is not a function of time, the Coulomb gauge seems to be the most expedient

choice for the particular problem. In the Coulomb gauge, the scalar potential acts instantaneously; however, the electric field still acts at the speed of light. Therefore, there is no problem of action-at-distance because the electric field is what is observed. However, we note the Coulomb gauge is a bad choice from the view of special relativity since in the Coulomb gauge $A^{\mu}$ is not a 4 -vector (it does not obey the transformation law of 4-vectors i.e. the Lorentz transformation).

\subsection{Pulsed Laser Fields}

In the Coulomb gauge, the potential form for Maxwell's equations reduce t0

$$
\begin{gathered}
\nabla^{2} V^{C}=-\frac{\rho}{\epsilon_{0}} \\
\nabla^{2} \mathbf{A}^{C}-\frac{1}{c^{2}} \frac{\partial^{2} \mathbf{A}^{C}}{\partial t^{2}}-\frac{1}{c^{2}} \nabla \frac{\partial V^{C}}{\partial t}=-\mu_{0} \mathbf{J}
\end{gathered}
$$

Another commonly employed electromagnetic gauge is the Lorenz gauge, which sets

$$
\boldsymbol{\nabla} \cdot \mathbf{A}^{L}+\frac{1}{c^{2}} \frac{\partial V^{L}}{\partial t}=0
$$


and reduces Maxwell's equations into the decoupled form

$$
\begin{aligned}
\nabla^{2} V^{L}-\frac{1}{c^{2}} \frac{\partial^{2} V^{L}}{\partial t^{2}} & =-\frac{\rho}{\epsilon_{0}} \\
\nabla^{2} \mathbf{A}^{L}-\frac{1}{c^{2}} \frac{\partial^{2} \mathbf{A}^{L}}{\partial t^{2}} & =-\mu_{0} \mathbf{J}
\end{aligned}
$$

When the source term is not a function of time (i.e. a stationary proton), the Coulomb gauge's scalar potential is not a function of time, and this gives a rather nice form to Maxwell's equations. Namely,

$$
\begin{aligned}
& \nabla^{2} V^{C} \quad=-\frac{\rho}{\epsilon_{0}} \\
& \nabla^{2} \mathbf{A}^{C}-\frac{1}{c^{2}} \frac{\partial^{2} \mathbf{A}^{C}}{\partial t^{2}}=-\mu_{0} \mathbf{J}
\end{aligned}
$$

The vector potential is an external field, thus remains time dependent. The sourceless Maxwell's equations are simply

$$
\begin{aligned}
& \nabla^{2} V=0 \\
& \nabla^{2} \mathbf{A}-\frac{1}{c^{2}} \frac{\partial^{2} \mathbf{A}}{\partial t^{2}}=0 \text {, }
\end{aligned}
$$

where we once again drop the superscript. The most general solution is,

$$
\mathbf{A}(\mathbf{r}, t)=\int \sum_{j} A_{j} \hat{\boldsymbol{\epsilon}}_{j}(\mathbf{k}) e^{i(\mathbf{k} \cdot \mathbf{r}-\omega t)} \frac{d^{3} k}{(2 \pi)^{3}}
$$

These equations permit wave solutions for the vector potential,

$$
\begin{aligned}
\mathbf{A}(\mathbf{r}, t) & =A\left(\mathbf{k}_{\omega} \cdot \mathbf{r}-\omega t\right) \hat{\boldsymbol{\epsilon}} \\
& =A\left(\omega\left(\frac{\hat{\mathbf{k}}_{\omega} \cdot \mathbf{r}}{c}-t\right)\right) \hat{\boldsymbol{\epsilon}}
\end{aligned}
$$

where $A$ is any well-behaved analytic function, the dispersion relation is $\omega=k c$, and for simplicity we have assumed linear polarization. A can be constructed from a linear 
combination of plane wave solutions,

$$
A\left(\mathbf{k}_{\omega} \cdot \mathbf{r}-\omega t\right)=\sum_{n} A_{n} \cos \left(\omega_{n}\left(\frac{\hat{\mathbf{k}}_{\omega} \cdot \mathbf{r}}{c}-t\right)\right) .
$$

That is, that any analytic function can be written as a linear combination of plane wave functions. For this reason, in this study we will focus on analysis consisting of plane wave solutions. We point out that there do exist some smooth functions (i.e. Bump functions) that happen to be non-analytic.

For a pulsed laser, the amplitude is localized in both space and time, and the separation between successive $\omega_{n}$ will approach zero as we add up the infinite sum. The infinite sum will effectively become an integral as we approach the continuum. Thus, a wave packet of light will be constructed by

$$
\mathbf{A}(\mathbf{r}, t)=\frac{1}{2 \pi} \int A\left(\omega^{\prime}\right) \cos \left(\omega^{\prime}\left(\frac{\hat{\mathbf{k}}_{\omega} \cdot \mathbf{r}}{c}-t\right)\right) d \omega^{\prime} \hat{\boldsymbol{\epsilon}}
$$

Assuming that the laser is truly monochromatic, then $A\left(\omega^{\prime}\right)$ reduces to $A_{0} 2 \pi \delta\left(\omega^{\prime}-\right.$ $\omega)$ leading directly to a plane wave solution with frequency $\omega$. That is, monochromatic light is completely localized in the frequency domain and completely delocalized in the time domain. However, for ultra short pulses the shape function $A\left(\omega^{\prime}\right)$ will have a spread that can extend over a range of frequencies. The spread in the wave packet is governed by the Fourier uncertainty principles. Namely,

$$
\begin{aligned}
\Delta \omega \Delta t & \geq \frac{1}{2} \\
\Delta k \Delta x & \geq \frac{1}{2},
\end{aligned}
$$

where $\Delta \omega$ is called the uncertainty or spread in the frequency, and is understood to be the standard deviation of the function $A(\omega)$. A typical Ti-Sapphire pulsed laser 
might have a pulse duration of about 70 femtoseconds. Using that duration for a rough approximation of the time uncertainty, namely $\Delta t \sim 70 \times 10^{-15} \mathrm{~s}$, we find that $\Delta f \approx 100 T H z$. This gives a color spread $\Delta \lambda \approx 200 \mathrm{~nm}$ (i.e. $\Delta f=c \lambda^{-2} \Delta \lambda$ ). This can be further reduced to around $\triangle \lambda \approx 30 \mathrm{~nm}$ by a technique in optics called "Modelocking." However, we can see from this example that a pulsed laser with a very short pulse time is not exactly monochromatic. Nevertheless, on the basis of the fact that close to the focus of the laser beam, the use of plane wave solutions is justified as long as the pulse lasts about ten optical cycles or more (i.e. $\tau \gg T$ or more precisely $\omega \Delta t \gg 1$ ), we will assume the laser is monochromatic. For a Ti-Sapphire laser, the pulse width is about $\tau \sim 70$ femtoseconds and the optical period is about $T \approx 2.7$ femtoseconds, which has well over ten optical cycles per pulse. On the other hand, with the more recent introduction of laser systems that can obtain attosecond pulses, pulse times can be of the order of the optical period. In the case of attosecond lasers, the pulse time must be dealt with explicitly. Ultra short pulses are discussed further in Appendix A.

In the dipole approximation, the vector potential is not a function of space. Thus,

$$
\mathbf{A}(t)=A(t) \cos (\omega t) \hat{\boldsymbol{\epsilon}}
$$

where $A(t)$ is the pulse shape function (i.e. Gaussian, sech or similar function) and is the Fourier pair of the function $A\left(\omega^{\prime}\right)$. In the case of non-monochromatic light with pulse with about ten optical cycles or more, the wave packet can be modeled by a frequency distribution function such as a Gaussian

$$
\begin{aligned}
A\left(\omega^{\prime}\right)=\left\langle\omega^{\prime} \mid A\right\rangle & =A_{0} 2 \pi \frac{1}{\sqrt{2 \pi} \Delta \omega} \exp \left(-\frac{1}{2 \Delta \omega^{2}}\left(\omega^{\prime}-\omega\right)^{2}\right) \\
& =A_{0} 2 \pi \frac{1}{\sqrt{2 \pi}} 2 \Delta t \exp \left(-\frac{(2 \Delta t)^{2}}{2}\left(\omega^{\prime}-\omega\right)^{2}\right) .
\end{aligned}
$$




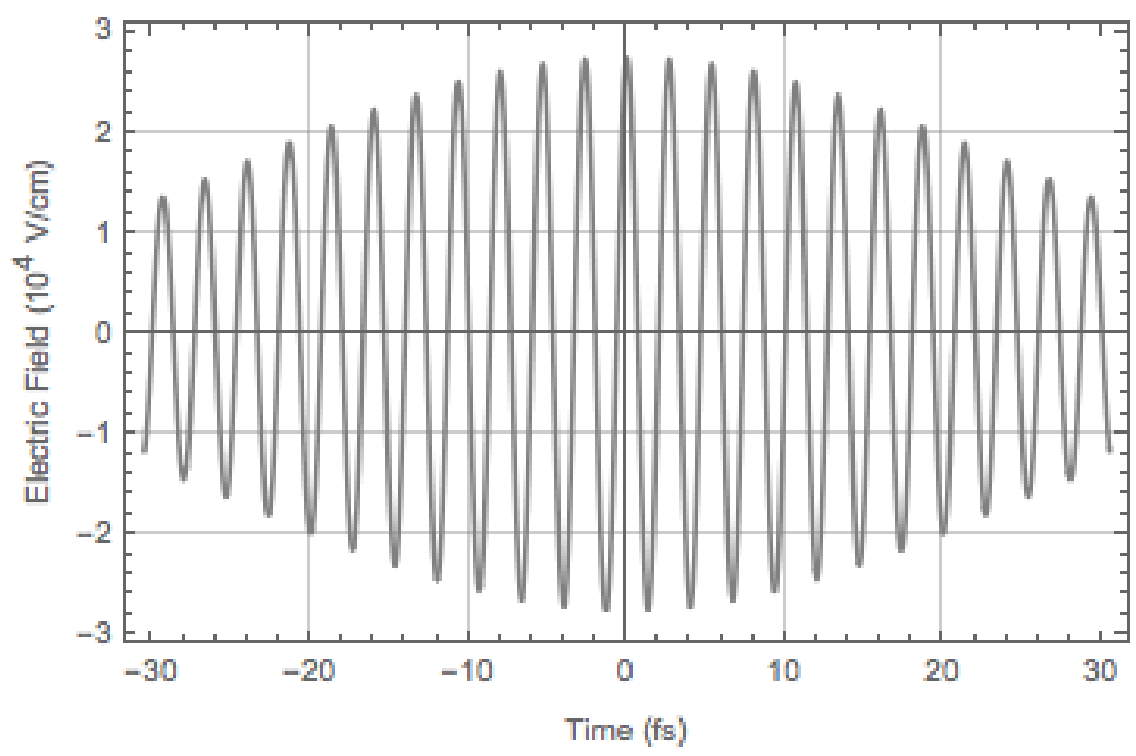

Figure 2.2: Electric Field vs. Time: Pulse period is $70 \mathrm{fs}$. Wavelength is $800 \mathrm{~nm}$. Notice, there are more than 10 optical periods within the pulse.

We find that the pulse shape function Fourier pair to be

$$
\begin{aligned}
A(t)=\langle t \mid A\rangle & =A_{0} \exp \left(-\frac{\Delta \omega^{2}}{2} t^{2}\right) \\
& =A_{0} \exp \left(-\frac{1}{2(2 \Delta t)^{2}} t^{2}\right) \\
& =A_{0} \exp \left(-\left(\frac{t}{\tau / 2}\right)^{2}\right),
\end{aligned}
$$

where $\tau=4 \sqrt{2} \Delta t$ is the spread in time of the pulse (pulse width) and the second lines have used the Fourier uncertainty principles.

\subsection{Two Common Dipole Gauges}

The second concept of gauge, which we will call the dipole gauge freedom, is

$$
\Psi^{\prime}(\mathbf{r}, t)=\exp \left[-i \frac{e}{\hbar} \Omega(\mathbf{r}, t)\right] \Psi(\mathbf{r}, t)
$$




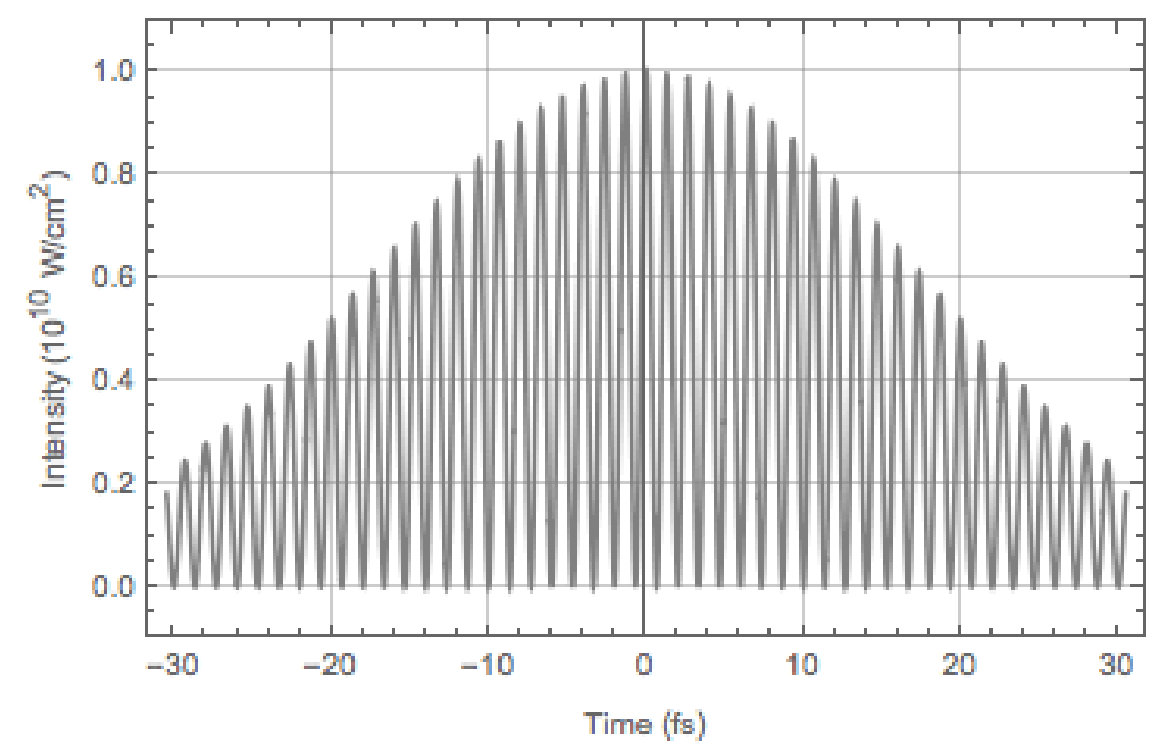

Figure 2.3: Intensity vs. Time: Pulse period is $70 \mathrm{fs}$. Wavelength is $800 \mathrm{~nm}$.

where we have changed from $\Lambda(\mathbf{r}, t)$ to $\Omega(\mathbf{r}, t)$ to emphasize that these dipole gauge transformations are in addition to the Coulomb gauge transformation. The dipole gauge freedom is only applicable within the dipole approximation $[32,33,34,35$, $36,37,38]$. Although a change by an overall phase constant in the wavefunction does not affect measurable quantities, a relative phase does matter. Therefore, the dipole gauge freedom is not a particular case of a unitary transformation. This misunderstanding is found throughout the literature and continues today. However, the choice to use the dipole approximation creates the conditions needed in order to correctly use the dipole gauge freedom. Measurable quantities (such as expectation values or transition probabilties) calculated in different dipole gauges must give the same value [39]. However, in practice differences arise because of approximations used in the calculations (e.g. approximate wavefunctions) leading some to incorrectly speculate that the one dipole gauge is more fundamental than another [40]. However, differences in measurable quantitites due to choice of dipole gauge will be small if accurate wavefunctions are used. 


\subsubsection{The Velocity Gauge}

The property of gauge invariance allows us to further simplify the TDSE by an appropriate choice of dipole gauge. We shall now see that, within the dipole approximation, the interaction term in the TDSE can be transformed into a somewhat more compact form.

We first show that the term $\mathbf{A}^{2}$ in the Hamiltonian can be eliminated via transforming the wavefunction by the time-dependent phase factor according to

$$
\Psi^{V}\left(\mathbf{r}_{1}, \mathbf{r}_{2}, \ldots, \mathbf{r}_{N}, t\right)=\exp \left[-\frac{i}{\hbar} \frac{e^{2} N}{2 m} \int_{-\infty}^{t} \mathbf{A}^{2}\left(t^{\prime}\right) d t^{\prime}\right] \Psi\left(\mathbf{r}_{1}, \mathbf{r}_{2}, \ldots, \mathbf{r}_{N}, t\right)
$$

where the vector potential, A, remember, is already in the Coulomb gauge. That is we have performed a dipole gauge transformtion such that

$$
\Omega^{V}\left(\mathbf{r}_{1}, \mathbf{r}_{2}, \ldots, \mathbf{r}_{N}, t\right)=\frac{e N}{2 m} \int_{-\infty}^{t} \mathbf{A}^{2}\left(t^{\prime}\right) d t^{\prime}
$$

With this choice, we have

$$
\begin{aligned}
& \mathbf{A}^{V}=\mathbf{A}+0 \\
& V^{V}=V-\frac{e N}{2 m} \mathbf{A}^{2} .
\end{aligned}
$$

We point out the fact that the dipole approximation makes the vector potential a function of time only, allowing the vector potential to remain un-transformed within this approximation. The resulting TDSE for the new wavefunction is

$$
i \hbar \frac{\partial}{\partial t} \Psi^{V}\left(\mathbf{r}_{1}, \mathbf{r}_{2}, \ldots, \mathbf{r}_{N}, t\right)=\left[H_{0}+\frac{e}{m} \mathbf{A}(t) \cdot \mathbf{P}\right] \Psi^{V}\left(\mathbf{r}_{1}, \mathbf{r}_{2}, \ldots, \mathbf{r}_{N}, t\right)
$$

where the two terms $\frac{e N}{2 m} \mathbf{A}^{2}$ have conveniently cancelled one another. The wavefunction has been transformed. However, the scalar and vector potential are in the good 
old Coulomb gauge. At this point some researchers believe that, as in the case for the electromagnetic gauge transformation, all the fields have been transformed into the new gauge. This is not true for the dipole gauge transformations. Here we have to use a mixture of Coulomb gauge and dipole gauge fields. In this example, the vector potential, $\mathbf{A}$, is in Coulomb gauge only. While the wavefunction, $\Psi^{V}$, is both Coulumb and dipole transformed. This confusion has caused many troubles within this field. This transformation is called the velocity gauge since the interaction Hamiltonian

$$
H_{\text {int }}^{V}(t)=\frac{e}{m} \mathbf{A}(t) \cdot \mathbf{P}
$$

couples the vector potential $\mathbf{A}(t)$ to the operator $\mathbf{P} / m$.

\subsubsection{The Length Gauge}

The TDSE in the dipole approximation can be transformed in another way that is also common in the literature. The wavefunction transformation is

$$
\Psi^{L}\left(\mathbf{r}_{1}, \mathbf{r}_{2}, \ldots, \mathbf{r}_{N}, t\right)=\exp \left[\frac{i}{\hbar} e \mathbf{A}(t) \cdot \mathbf{R}\right] \Psi\left(\mathbf{r}_{1}, \mathbf{r}_{2}, \ldots, \mathbf{r}_{N}, t\right)
$$

where

$$
\mathbf{R}=\sum_{i=1}^{N} \mathbf{r}_{i}
$$

is the sum of the coordinates of the $N$ electrons. Thus we have performed a dipole gauge transformation such that

$$
\Omega^{L}\left(\mathbf{r}_{1}, \mathbf{r}_{2}, \ldots, \mathbf{r}_{N}, t\right)=-\mathbf{A}(t) \cdot \mathbf{R}
$$


With this choice, we have

$$
\begin{aligned}
\mathbf{A}^{L} & = & \mathbf{A}-A(t) \boldsymbol{\nabla}(\hat{\boldsymbol{\epsilon}} \cdot \mathbf{R}) \\
& = & \mathbf{A}-A(t) \hat{\boldsymbol{\epsilon}} \\
& = & 0
\end{aligned}
$$

and

$$
\begin{aligned}
V^{L} & =V-\frac{\partial}{\partial t}(\mathbf{A}(t) \cdot \mathbf{R}) \\
& =V-\left(\frac{\partial \mathbf{A}}{\partial t} \cdot \mathbf{R}\right) \\
& =V+\mathbf{E}_{\text {trans }}(t) \cdot \mathbf{R} .
\end{aligned}
$$

Again, we point out the fact that the dipole approximation makes the vector potential a function of time only, allowing the vector potential to transform to zero within this dipole transformation (i.e. length gauge). The TDSE for this transformation is

$$
i \hbar \frac{\partial}{\partial t} \Psi^{L}\left(\mathbf{r}_{1}, \mathbf{r}_{2}, \ldots, \mathbf{r}_{N}, t\right)=\left[H_{0}+e \mathbf{E}_{\text {trans }}(t) \cdot \mathbf{R}\right] \Psi^{L}\left(\mathbf{r}_{1}, \mathbf{r}_{2}, \ldots, \mathbf{r}_{N}, t\right)
$$

which is said to be in the length gauge because the interaction Hamiltonian

$$
H_{\text {int }}^{L}(t)=e \mathbf{E}_{\text {trans }}(t) \cdot \mathbf{R}
$$

couples the electric field $\mathbf{E}_{\text {trans }}(t)$ to the operator $\mathbf{R}$. Notice again the fact that the fields (i.e. $V$ and $\mathbf{A}$ ) in the Hamiltonian are the Coulomb gauge fields, not the length gauge fields. However, the wavefunctions are transformed into the length gauge. While the electromatic field, $\mathbf{E}$, is gauge independent, the choice of the Coulomb gauge for the electromagnetic gauge freedom means the field must be transverse. This mixing of guages simplifies calculation, while at the same time adds to so much confusion. In some sense, one could look at $V_{\text {trans }}=\mathbf{E}_{\text {trans }}(t) \cdot \mathbf{R}$ as a kind of potential 

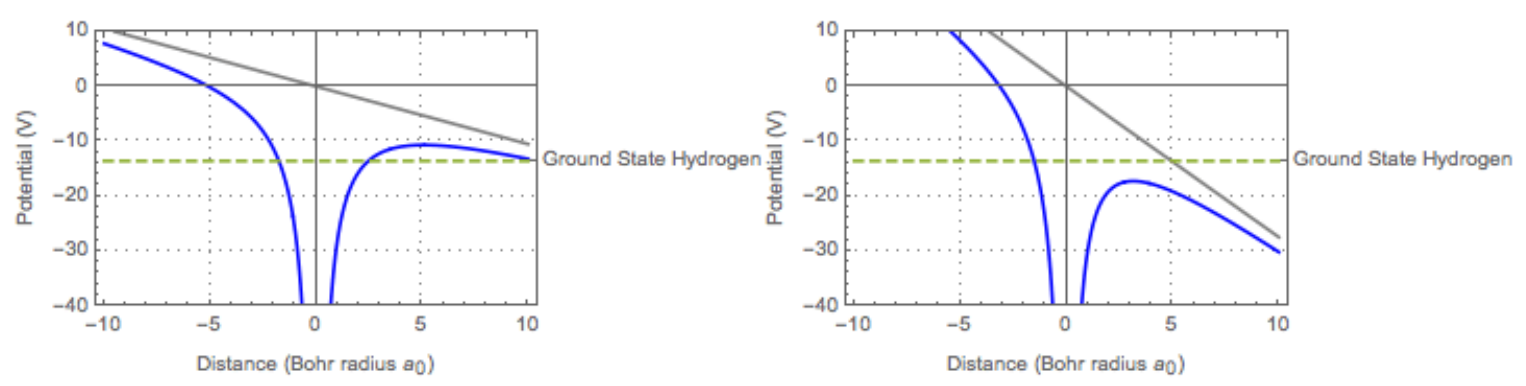

Figure 2.4: Potential vs. Distance: In the length gauge, the interaction acts like a scalar potential that modifies the Coulomb potential. For this reason, many researchers like to use the length gauge.

that raises and lowers the Coulomb potential. However, just as in the case of picking the Coulomb gauge in Section 2.6 makes the potential, $V$, act instantaneously over distance (faster than light travel?!), the length gauge picture is not an entirely faithful view of the actual physical process. This unfaithful view is sometimes referred to as Tunneling Ionization. By introducing the electric dipole moment operator

$$
\mathbf{D}=\sum_{i=1}^{N}(-e) \mathbf{r}_{i}=-e \mathbf{R} .
$$

The interaction Hamiltonian can be written in another common form

$$
H_{\text {int }}^{L}(t)=-\mathbf{E}_{\text {trans }}(t) \cdot \mathbf{D} .
$$

Some like to claim the length gauge is the "real gauge," "gauge-invariant gauge" and/or "correct gauge" because it has a form with an explicit electric field rather than the "auxiliary functions" $V$ and $\mathbf{A}$, but these statements merely show the ignorance of the user to the fact that the length gauge is derived fundamentally from a Hamiltonian function created explicitly of $V$ and $\mathbf{A}$, and the length gauge is an approximation (dipole approx.) to this more fundamental Hamiltonian [41]. Although there are many other possible dipole gauge transformations that are of particular interest to 
specific areas of research, we shall have no need of them.

\subsection{Interaction of a Single Electron with an EM Field}

Let us solve the wavefunction for the simple case of a single "free" electron in the presence of a field under the assumption that the vector potential is only a function of time (dipole approximation). The electron's motion is described by the TDSE

$$
i \hbar \frac{\partial}{\partial t} \chi(\mathbf{r}, t)=\frac{1}{2 m}(\mathbf{p}+e \mathbf{A}(t))^{2} \chi(\mathbf{r}, t) .
$$

We will look for solutions having the form

$$
\chi(\mathbf{r}, t)=\exp (i \mathbf{k} \cdot \mathbf{r}) f(t),
$$

where $\mathbf{k}$ is related to the momentum of the electron by $\mathbf{p}=\hbar \mathbf{k}$. Plugging into the TDSE,

$$
\begin{array}{rlrl}
i \hbar \frac{\partial}{\partial t}(\exp (i \mathbf{k} \cdot \mathbf{r}) f(t)) & = & \frac{1}{2 m}(\mathbf{p}+e \mathbf{A}(t))^{2}(\exp (i \mathbf{k} \cdot \mathbf{r}) f(t)) \\
i \hbar \exp (i \mathbf{k} \cdot \mathbf{r}) \frac{\partial}{\partial t} f(t) & = & & \left(\frac{\mathbf{p}^{2}}{2 m}+\frac{e}{2 m} \mathbf{p} \cdot \mathbf{A}(t)+\frac{e}{2 m} \mathbf{A}(t) \cdot \mathbf{p}+\frac{e^{2}}{2 m} \mathbf{A}^{2}(t)\right) \exp (i \mathbf{k} \cdot \mathbf{r}) f(t) \\
i \hbar \exp (i \mathbf{k} \cdot \mathbf{r}) \frac{\partial}{\partial t} f(t) & = & \left(\frac{\hbar^{2}}{2 m} \nabla^{2}-i \frac{e \hbar}{2 m} \nabla \cdot \mathbf{A}(t)-i \frac{e \hbar}{2 m} \mathbf{A}(t) \cdot \boldsymbol{\nabla}+\frac{e^{2}}{2 m} \mathbf{A}^{2}(t)\right) \exp (i \mathbf{k} \cdot \mathbf{r}) f(t) \\
i \hbar \exp (i \mathbf{k} \cdot \mathbf{r}) \frac{\partial}{\partial t} f(t) & = & \left(\frac{\hbar^{2} k^{2}}{2 m}+\frac{e \hbar}{2 m} \mathbf{k} \cdot \mathbf{A}(t)+\frac{e \hbar}{2 m} \mathbf{A}(t) \cdot \mathbf{k}+\frac{e^{2}}{2 m} \mathbf{A}^{2}(t)\right) \exp (i \mathbf{k} \cdot \mathbf{r}) f(t) \\
i \hbar \exp (i \mathbf{k} \cdot \mathbf{r}) \frac{\partial}{\partial t} f(t) & = & \left(\frac{\hbar^{2} k^{2}}{2 m}+\frac{e \hbar}{m} \mathbf{k} \cdot \mathbf{A}(t)+\frac{e^{2}}{2 m} \mathbf{A}^{2}(t)\right) \exp (i \mathbf{k} \cdot \mathbf{r}) f(t) \\
i \hbar \frac{\partial}{\partial t} f(t) & = & \left(\frac{\hbar^{2} k^{2}}{2 m}+\frac{e \hbar}{m} \mathbf{k} \cdot \mathbf{A}(t)+\frac{e^{2}}{2 m} \mathbf{A}^{2}(t)\right) f(t) .
\end{array}
$$

Note that although this result can be derived using the less restrictive Coulomb gauge condition, the fact that the vector potential is only a function of time is sufficient at this point. What would happen had we not used the the Coulomb gauge and dipole approximation? Well, in that case $\mathbf{A}(\mathbf{r}, t)$ would still be a function of space, and 
we would get factors $k_{\omega}$ from derivatives of $\mathbf{A}(\mathbf{r}, t)$. That is, we would find that the momentum and the field no longer commute,

$$
[\mathbf{p}, \mathbf{A}] \neq 0 .
$$

Had we used the version with factors of $k_{\omega}$, we would find out later that picking up those factors would hinder our ability to find nice solutions later on (i.e. using integration by parts in the Strong Field analysis.). It seems, at the very least, we should use the Coulomb gauge to prevent the appearance of factors of $k_{\omega}$. If we would like a gauge free analysis, then it is probably best to use a fully relativitic theory from the start.

The equation is readily solved for $f(t)$ to give

$$
\begin{aligned}
f(t)= & C \exp \left[-\frac{i}{\hbar}\left(\frac{\hbar^{2} k^{2}}{2 m} t+\int\left(\frac{e \hbar}{m} \mathbf{k} \cdot \mathbf{A}(t)+\frac{e^{2}}{2 m} \mathbf{A}^{2}(t)\right) d t\right)\right] \\
= & C \exp \left[-\frac{i}{\hbar}\left(E_{k} t+\int V_{i n t}\left(t^{\prime}\right) d t^{\prime}\right)\right]
\end{aligned}
$$

where $E_{k}=\hbar^{2} k^{2} /(2 m)$ is the electron's kinetic energy and

$$
V_{\text {int }}(t)=\frac{e \hbar}{m} \mathbf{k} \cdot \mathbf{A}(t)+\frac{e^{2}}{2 m} \mathbf{A}^{2}(t)
$$

is the interaction potential of the single electron. Thus, the Volkov solution for a single "free" electron in the presence of a field is [42]

$$
\chi(\mathbf{r}, t)=\frac{1}{\sqrt{\mathcal{V}}} \exp (i \mathbf{k} \cdot \mathbf{r}) \exp \left[-\frac{i}{\hbar}\left(E_{k} t+\int V_{\text {int }}\left(t^{\prime}\right) d t^{\prime}\right)\right]
$$

where $\mathcal{V}$ is the volume and we have chosen the $C$ such that the inner product is normalized to unity. Note, within each dipole gauge the Volkov solution takes on the 
form corresponding to that dipole gauge interaction potential. 


\section{Chapter 3}

\section{Rates, Cross-Sections, and Detailed Balancing}

\subsection{Rate Equation}

The rate equation for our system is

$$
\frac{d \mathcal{N}_{H^{-}}}{d t}=\Gamma_{S A} \mathcal{N}_{H}-\Gamma_{P D} \mathcal{N}_{H^{-}}+\Gamma_{R A} \mathcal{N}_{H}
$$

or

$$
\frac{d n_{H^{-}}}{d t}=\alpha_{S A} n_{e} n_{H}-\alpha_{P D} n n_{H^{-}}+\alpha_{R A} n_{e} n_{H}
$$

The rate of stimulated radiative attachment of an electron, $\Gamma_{S A}$, is sometimes referred to "as the rate of stimulated emission of a photon." Although one can have stimulated emission from a bound, yet excited state, electron, we are only concerned with the transition between continuous and bound states. The rate of photodetachment of an electron, $\Gamma_{P D}$, is sometimes referred to as "the rate of absorption of a photon." Although one can have absorption that does not detach an electron, we are only concerned with the ones that do. Finally, rate of radiative attachment, $\Gamma_{R A}$, is closely related to the rate of spontaneous emission. Again, we are concerned here with the transition between continuous and bound states. Spontaneous emission is extremely small in comparsion with stimulated radiative attachment in strong laser fields. The subject of spontaneous radiative attachment has been treated by Keating, Charlton 
and Straton $[24,25]$. In the second version of the rate equation, $n_{i}=\mathcal{N}_{i} / \mathcal{V}$ is the number density and $\alpha_{S A}=\Gamma_{S A} / n_{e}$ (not to be confused with $\alpha$, the fine structure constant) is called "the rate coefficient or constant." It can be shown that $\alpha_{i}=$ $v \sigma_{i}$, where $v$ is the velocity of the electron. We note that $\alpha_{S A}(I)$ depends on the intensity (photon number) of the laser field, while the other rate coefficients are, in fact, constants with respect to intensity.

Neumann et al. also put estimates on rates of re-ionization [43]. They claim there is an upper limit on photon number (i.e. laser intensity). The argument says that the cross-section for photodetachment is $\sigma_{P D} \approx 10^{-17} \mathrm{~cm}^{2}$. The photodetachment rate is

$$
\Gamma_{P D}=\Phi \sigma_{P D},
$$

where $\Phi$ is the flux (of photons). If the atom is exposed to the laser field for a time $\tau$, then the relation

$$
\Gamma_{P D} \tau=\Phi \sigma_{P D} \tau \leq 1
$$

must hold in order to ensure that the atom formed is not re-ionized. This last statement is somewhat wanting in explanation. Thus,

$$
\Phi \leq \frac{1}{\sigma_{P D} \tau},
$$

with the value of $\tau \approx 70$ femtoseconds, we find

$$
\Phi \lesssim 10^{30} \frac{\text { photons }}{\mathrm{cm}^{2} \mathrm{~s}}
$$

or

$$
I \lesssim 10^{11} \frac{W}{\mathrm{~cm}^{2}} .
$$


However, this estimate does not take into account the rate of attachment. The rate of attachment is a competing process that is happening during the same laser pulse. A more accurate analysis should include the rate of attachment.

Consider instead the case where the stimulated attachment rate coefficient is linear with intensity, that is $\alpha_{S A}(n)=\beta_{S A} n$, as is the case for intensities within first order perturbation theory. The argument can be easily generalized to any arbitrary function. Then,

$$
\frac{d n_{H^{-}}}{d t}=\left(\beta_{S A} n_{e} n_{H}-\alpha_{P D} n_{H^{-}}\right) n
$$

and we find the condition for production of (anti)hydrogen ions to be

$$
\beta_{S A} n_{e} n_{H}>\alpha_{P D} n_{H^{-}}
$$

Notice that the photon density does affect the rate of production but does not have an upper limit at which re-ionization dominates attachment. This is a result of the rate coefficient being linear with intensity. This analysis seems to contradict the idea of an upper limit on the laser intensity as a result of the re-ionization process that Neumann claims. Perhaps, the Neumann limit has more to do with the breakdown of first order perturbation than re-ionization.

Since in our case, the reaction is antihydogen limited, $\mathcal{N}_{H} \approx 10^{4}$, we will need to have a high positron density, $n_{e}$, and actively remove the product, thereby making $n_{H^{-}}$small. Although not ideal, we can always allow the system to reach equilibrium. In this case,

$$
n_{H^{-}}=\frac{\beta_{S A}}{\alpha_{P D}} n_{e} n_{H}
$$

and we once again see the need to have a high positron density. Notice that the equilibrium is not a function of photon density. This means we can create a large 
photon density (i.e strong intensity laser field) to increase the rates of attachment and, more importantly, when we can turn off the laser field, the product will remain.

\subsection{Detailed Balancing}

It has become standard to use the principle of detailed balancing to find the cross sections for attachment. In order to derive the principle of detailed balancing, we consider a box containing electrons, hydrogen atoms, hydrogen ions, and photons. Ignoring spontanious emission, which is very small in comparsion to the laser field, at equilibrium we find

$$
\Gamma_{S A} \mathcal{N}_{H}=\Gamma_{P D} \mathcal{N}_{H^{-}}
$$

We will need to derive a scattering cross-section. First, note that the power is the energy times rate, $P=\Gamma E$, and the intensity is power per cross-section, $I=P / \sigma=$ $\Gamma E / \sigma$ and flux $\Phi=\Gamma / \sigma=I / E$. This gives,

$$
\sigma=\frac{\Gamma E}{I}=\frac{\Gamma}{\Phi} .
$$

Changing from rates to cross-sections we find

$$
v \sigma_{S A} \mathcal{N}_{H} \mathcal{N}_{e}=c \sigma_{P D} \mathcal{N}_{H^{-}} \mathcal{N}
$$

This equation holds if we replace the number of particles by the density of states for each particle. (See Bethe p. 61 for this "hand waving" derivation [44].) We will make this more precise at a later point. Thus,

$$
v \sigma_{S A}[2]\left[\mathcal{N}_{e} 2 \frac{\mathcal{V}}{(2 \pi)^{3} \hbar^{3}} p^{2} d p\right]=c \sigma_{P D}[1]\left[\mathcal{N} 2 \frac{\mathcal{V}}{(2 \pi)^{3} \hbar^{3}}\left(\frac{\hbar \omega}{c}\right)^{2} \frac{\hbar}{c} d \omega\right]
$$


where the factors of ones and twos are due to the spin or polarization states. This becomes

$$
\sigma_{S A} \mathcal{N}_{e} p^{2}=\sigma_{P D} \mathcal{N} \frac{1}{2}\left(\frac{\hbar \omega}{c}\right)^{2}
$$

Written this way

$$
\sigma_{S A}=\frac{\mathcal{N}}{\mathcal{N}_{e}} \frac{1}{2} \frac{\left(\frac{\hbar \omega}{c}\right)^{2}}{p^{2}} \sigma_{P D}
$$

With only one electron this simplifies to

$$
\sigma_{S A}=\mathcal{N} \frac{1}{2} \frac{\left(\frac{\hbar \omega}{c}\right)^{2}}{p^{2}} \sigma_{P D}
$$

This is the Principle of Detailed Balancing. It has become standard to calculate cross sections (or rates) for photodetachment, and use the principle of detailed balancing to find the cross sections for attachment. We note that for spontaneous radiative attachment one finds,

$$
\sigma_{R A}=\frac{1}{\mathcal{N}_{e}} \frac{1}{2} \frac{\left(\frac{\hbar \omega}{c}\right)^{2}}{p^{2}} \sigma_{P D}
$$

With only one electron, this simplifies to

$$
\sigma_{R A}=\frac{1}{2} \frac{\left(\frac{\hbar \omega}{c}\right)^{2}}{p^{2}} \sigma_{P D}
$$

for each single electron. The difference between stimulated and spontaneous emission has its origins in the fact that in second quantization

$$
\left|\left\langle\mathcal{N}+1\left|a^{\dagger}\right| \mathcal{N}\right\rangle\right|^{2}=|\langle\mathcal{N}+1|\sqrt{\mathcal{N}+1}| \mathcal{N}+1\rangle|^{2}=\mathcal{N}+1
$$

where the $\mathcal{N}$ is associated with stimulated emission and 1 is associated with spontaneous emission such that $\Gamma_{S A}=\mathcal{N} \Gamma_{R A}$ and $\alpha_{S A}=\mathcal{N} \alpha_{R A}$. 


\section{Chapter 4}

\section{Perturbation Theory}

\subsection{First Order Perturbation}

The first step in time-dependent perturbation theory is to write the Hamiltonian as

$$
H(t)=H_{0}+H^{\prime}(t)
$$

where the time evolution induced by $H_{0}$ can be solved exactly and the perturbation $H^{\prime}(t)$ is (in some sense) small. Because the unperturbed basis is a complete set, we can always expand the full time-dependent wavefunction in terms of the unperturbed basis

$$
\Psi(\mathbf{r}, t)=\langle\mathbf{r} \mid \Psi(t)\rangle=\sum_{n} d n\langle\mathbf{r} \mid n\rangle\langle n \mid \Psi(t)\rangle=\sum_{n} d n c_{n}(t) \psi_{n}^{(0)}(\mathbf{r})
$$

where we have used the identity $1=\sum d n|n\rangle\langle n|, n$ being a general label for the basis states, and $\langle\mathbf{r} \mid n\rangle=\psi_{n}^{(0)}(\mathbf{r}),\langle n \mid \Psi(t)\rangle=c_{n}(t)$. Here our familiar notation for the Hamiltonian translates to $\left\langle\mathbf{r}|H(t)| \mathbf{r}^{\prime}\right\rangle=\int d^{3} x H\left(\mathbf{r}^{\prime}, t\right) \delta^{3}\left(\mathbf{r}-\mathbf{r}^{\prime}\right)$. When the the unperturbed Hamiltonian has bound states (i.e. Coulomb potential) then the 
spectrum will have both discrete states and continuum states

$$
\Psi(\mathbf{r}, t)=\sum_{n} c_{n}(t) \psi_{n}^{(0)}(\mathbf{r})+\int d n c_{n}(t) \psi_{n}^{(0)}(\mathbf{r})
$$

where the sum is over $n$ in the discrete parts of the spectrum $(n \rightarrow n l m$ for Hydrogen), the integral is over $n$ in the continuous parts of the spectrum $\left(d n \rightarrow d^{3} n\right.$ in $3 \mathrm{D}$, $\left.d^{3} p=\frac{\hbar^{3}(2 \pi)^{3}}{\mathcal{V}} d^{3} n\right)$. We note the units of the coefficients $c_{n}(t)$ are dimensionless. By convention, the units of the wavefunctions are $1 / \sqrt{\mathcal{V}}$. Now $c_{n}(t)$ changes with time because of both $H_{0}$ and $H^{\prime}$. Ignoring $H^{\prime}$ for the moment, we find

$$
c_{n}(t)=c_{n}(0) e^{-i E_{n}^{(0)} t / \hbar}
$$

This gives us the time dependence of the unperturbed system. Knowing this, we now seek the time dependence of the perturbation. Expanding the wavefunction such that the time dependence of the unperturbed system is factored out, allows us to solve for the time dependence of the perturbation. Thus, we expand the wavefunction as

$$
\Psi(\mathbf{r}, t)=\sum d_{n}(t) e^{-i E_{n}^{(0)} t / \hbar} \psi_{n}^{(0)}(\mathbf{r})
$$

where $d_{n}(t)$ is a new coefficiant that only contains the "dynamic" perturbed time dependence. This idea of factoring out the "kinematic" time dependence is an example of what is called the "Interaction Picture." Next, we insert the expanded wavefunction into the time-dependent Schrödinger equation, multiplying by $\psi_{m}^{(0) *}(\mathbf{r}) e^{i E_{m}^{(0)} t / \hbar}$ and integrating over all space. Solving for $d_{m}(t)$ we find the "exact solution" to be

$$
d_{m}(t)=\delta_{m n}-\frac{i}{\hbar} \sum_{n} \iint \psi_{m}^{(0) *}(\mathbf{r}) H^{\prime}(t) \psi_{n}^{(0)}(\mathbf{r}) e^{i \omega_{m n} t} d_{n}(t) d^{3} x d t
$$


where $\omega_{m n}=\left(E_{m}^{(0)}-E_{n}^{(0)}\right) / \hbar$. We note that this is not really a solution, rather an integral equation; the solution we seek is also in the integral. Since in the distant past the perturbation was off and in the far future the perturbation is also off, we can say that in both cases the system is in an eigenstate of the unperturbed Hamiltonian (i.e. $\left.\Psi(\mathbf{r},-\infty)=\psi_{i}^{(0)}(\mathbf{r}), \Psi(\mathbf{r}, \infty)=\psi_{f}^{(0)}(\mathbf{r})\right)$. Suppose the system starts in the inital state, $\Psi(\mathbf{r},-\infty)=\psi_{i}^{(0)}(\mathbf{r})$, in the distant past. We will make the zeroth order approximation, that is no perturbation $\left(H^{\prime}(t)=0\right)$. In this case, the state remains unchanged, $\Psi(\mathbf{r}, \infty)=\psi_{i}^{(0)}(\mathbf{r})$, after the (un)perturbation. Therefore, the wavefunction is time independent and we can see that to zeroth order

$$
d_{m}^{(0)}(t)=\delta_{m n}
$$

Plugging $d_{i}(t)=\delta_{i n}$ in to the right hand side, we find the first order approximation to be

$$
d_{f}(t) \approx \delta_{f i}-\frac{i}{\hbar} \iint \psi_{f}^{(0) *}(\mathbf{r}) H^{\prime}(t) \psi_{i}^{(0)}(\mathbf{r}) e^{i \omega_{f i} t} d^{3} x d t
$$

where we have changed notation to coincide with the final and initial state. The first order approximation is

$$
d_{f}^{(1)}(t)=-\frac{i}{\hbar} \iint \psi_{f}^{(0) *}(\mathbf{r}) H^{\prime}(t) \psi_{i}^{(0)}(\mathbf{r}) e^{i \omega_{f i} t} d^{3} x d t
$$

Of course, we could go on to the second order approximations by plugging this first order approximation into the "exact solution,"

$$
\begin{gathered}
d_{f}^{(2)}(t)= \\
\left.\left(\frac{i}{\hbar}\right) \sum_{n}^{2} \iint \psi_{f}^{(0) *}(\mathbf{r}) H^{\prime}(t) \psi_{n}^{(0)}(\mathbf{r}) e^{i \omega_{f n} t}\left[\iint \psi_{n}^{(0) *}\left(\mathbf{r}^{\prime}\right) H^{\prime}\left(t^{\prime}\right) \psi_{i}^{(0)}\left(\mathbf{r}^{\prime}\right) e^{i \omega_{n i} t^{\prime}} d^{3} x^{\prime} d t^{\prime}\right] d^{3} x d t .10\right)
\end{gathered}
$$


However, the second order solution becomes rather messy to work with and the extra term is, in most cases, not needed.

\subsection{Periodic Perturbation}

Now we will consider the system subjected to a periodic perturbation

$$
H^{\prime}(t)=H^{\prime} e^{-i \omega t}
$$

where $H^{\prime}(t)$ now has explicit periodic dependence on time and $H^{\prime}$ is the time independent part. It is important to remember that the perturbation is at some time in the past turned off as well as in the future turned off. This could be included explicitly by a pulse shape function (e.g. $f(t)=\exp \left(-t^{2} /(\tau / 2)^{2}\right)$ or $\left.f(t)=\operatorname{rect}(t / \tau)\right)$, where perturbation time $\tau$ is large compared to the optical period. These functions are zero at times in the remote past as well as zero at times in the far future. Though the pulse shape function is mathematically more rigorous, it is also more tedious. For brevity, we will not use the pulse shape function here. For those interested, see Appendix B. Plugging the periodic perturbation and noticing that the final state is not the same as the initial state (i.e. $\delta_{f i}=0$ ), we find

$$
d_{f}(t)=-\frac{i}{\hbar} \int \psi_{f}^{(0) *}(\mathbf{r}) H^{\prime} \psi_{i}^{(0)}(\mathbf{r}) d^{3} x \int_{t_{0}}^{t_{f}} e^{i\left(\omega_{f i}-\omega\right) t} d t
$$

Let us have the perturbation starting at $t_{0}=-\tau / 2$ and ending at $t_{f}=\tau / 2$ for symmetry. For long perturbation times,

$$
d_{f}(\tau \rightarrow \infty)=-2 \pi \frac{i}{\hbar} \int \psi_{f}^{(0) *}(\mathbf{r}) H^{\prime} \psi_{i}^{(0)}(\mathbf{r}) d^{3} x \delta\left(\omega_{f i}-\omega\right)
$$


The transition probability is defined as

$$
P_{f i}(\tau) \equiv\left|\int \Psi^{*}(\mathbf{r}, \tau) \Psi(\mathbf{r},-\infty) d^{3} x\right|^{2}=\left|d_{f}(\tau)\right|^{2}
$$

For very long perturbation times, we find

$$
P_{f i}(\tau \rightarrow \infty)=\frac{4 \pi^{2}}{\hbar^{2}}\left|\int \psi_{f}^{(0) *}(\mathbf{r}) H^{\prime} \psi_{i}^{(0)}(\mathbf{r}) d^{3} x\right|^{2} \delta\left(\omega_{f i}-\omega\right) \delta\left(\omega_{f i}-\omega\right)
$$

Use the fact,

$$
f(x) \delta\left(x-x_{0}\right)=f\left(x_{0}\right) \delta\left(x-x_{0}\right)
$$

and assume that the perturbation time, although large, is finite. After all we cannot wait forever to make our measurement. We find

$$
\begin{aligned}
P_{f i}(\tau \rightarrow \infty) & =\frac{4 \pi^{2}}{\hbar^{2}}\left|\int \psi_{f}^{(0) *}(\mathbf{r}) H^{\prime} \psi_{i}^{(0)}(\mathbf{r}) d^{3} x\right|^{2} \delta(0) \delta\left(\omega_{f i}-\omega\right) \\
& =\frac{4 \pi^{2}}{\hbar^{2}}\left|\int \psi_{f}^{(0) *}(\mathbf{r}) H^{\prime} \psi_{i}^{(0)}(\mathbf{r}) d^{3} x\right|^{2} \frac{\tau}{2 \pi} \delta\left(\omega_{f i}-\omega\right) .
\end{aligned}
$$

Although the last few steps may seem lax to a strict mathematician, they do display the brevity in using generalized functions in the physical sciences. Most importantly, generalized functions give us the correct results. The rate of transition can be expressed as

$$
\Gamma_{f i}=\frac{P_{f i}(\tau)}{\tau}
$$

Thus, the rate of transition is

$$
\Gamma_{f i}=\frac{2 \pi}{\hbar}\left|\int \psi_{f}^{(0) *}(\mathbf{r}) H^{\prime} \psi_{i}^{(0)}(\mathbf{r}) d^{3} x\right|^{2} \delta\left(E_{f}^{(0)}-E_{i}^{(0)}-\hbar \omega\right)
$$

Though found first by Dirac, then Wentzel, it was popularized by Fermi and is called Fermi's Golden Rule. We note that for very short perturbation times $\delta\left(E_{f}^{(0)}-E_{i}^{(0)}-\right.$ 
$\hbar \omega) \rightarrow f\left(E_{f}^{(0)}-E_{i}^{(0)}-\hbar \omega\right)$, where $f$ has a spread in energy of $\Delta E \gtrsim \hbar /(2 \Delta t)$. This is one example of peak-broadening.

Now we turn our attention to the delta function $\delta\left(E_{f}^{(0)}-E_{i}^{(0)}-\hbar \omega\right)$. For any argument of the delta function

$$
\delta[g(x)]=\sum_{i} \frac{\delta\left(x-x_{i}\right)}{\left|g^{\prime}\left(x_{i}\right)\right|}
$$

Thus,

$$
\delta\left(x^{2}-a^{2}\right)=\frac{1}{2 a}[\delta(x-a)+\delta(x+a)]
$$

Using this

$$
\delta\left(E_{f}^{(0)}-E_{i}^{(0)}-\hbar \omega\right)=\frac{m}{p} \delta\left(p-\sqrt{2 m\left(E_{i}^{(0)}+\hbar \omega\right)}\right),
$$

where we have kept only the term that takes the system from a lower energy state to a higher one. Thus, the rate of transition is

$$
\Gamma_{f i}=\frac{2 \pi}{\hbar}\left|\int \psi_{f}^{(0) *}(\mathbf{r}) H^{\prime} \psi_{i}^{(0)}(\mathbf{r}) d^{3} x\right|^{2} \frac{m}{p} \delta\left(p-\sqrt{2 m\left(E_{i}^{(0)}+\hbar \omega\right)}\right)
$$

The detector is usually only sensitive over a small cross section of space and is usually insensitive to the magnitude of the momentum of the ejected electron. Thus, it is necessary to integrate over the momentum space of the ejected electron, that is $d^{3} p=\frac{\hbar^{3}(2 \pi)^{3}}{\mathcal{V}} d^{3} n$, so that

$$
\begin{gathered}
\Gamma_{P D}=\sum_{f} \Gamma_{f i}=\int \Gamma_{f i} d^{3} n_{f}= \\
\iint\left(\frac{2 \pi}{\hbar}\left|\int \psi_{f}^{(0) *}(\mathbf{r}) H^{\prime} \psi_{i}^{(0)}(\mathbf{r}) d^{3} x\right|^{2} \frac{m}{p} \delta\left(p-\sqrt{2 m\left(E_{i}^{(0)}+\hbar \omega\right)}\right)\right) g_{P D} \frac{\mathcal{V}}{(2 \pi)^{3} \hbar^{3}} p^{2} d p d \Omega
\end{gathered}
$$

where $g_{P D}$ is the factor accounting for spin and polarization states. 
Up until this point, we have not been explicit about spin. However, at this point we need to point out that the spin states do affect whether an electric dipole transition will occur. The electric dipole transition selection rules are summed up as follows:

$$
\begin{gathered}
\Delta l= \pm 1 \\
\Delta m=0, \pm 1 \\
\Delta s=0
\end{gathered}
$$

Firstly, if our detector is not sensitive to spin, we need to include factor of two for the ejected electron since we will detect both spins without distinguishing between them. We need another factor of two for the two spin states remaining for the Hydrogen atom. However, we also need to account for the unknown spin state of the initial system. The initial state is the three-particle system with a mix of half total-spin coupled $s=0$ electron pairs and half $s=1$ electron pairs. Since electric dipole selection rules dictate that $\Delta s=0, s=0$ initial system states will interact only with $s=0$ final state systems and the same for $s=1$ initial and final state systems. That means that we overcounted the rate by a factor of two. Finally, there is the polarization of the photon. We do not know the polarization of the photon that interacted with the electron. Therefore, we overcounted by a factor of two again.

In summary,

$$
\begin{gathered}
g_{P D}=(\text { sum final spin states })(\text { average initial spin states })= \\
\left(2 s_{\text {electron }}+1\right)\left(2 s_{\text {Hydrogen }}+1\right) \frac{1}{\text { polarization (spin states target })}=(2)(2) \frac{1}{2(2)}=1
\end{gathered}
$$


Thus, the rate of transition per solid angle is

$$
\frac{d \Gamma_{P D}}{d \Omega}=\frac{\mathcal{V} m}{(2 \pi)^{2} \hbar^{4}} p\left|\int \psi_{f}^{(0) *}(\mathbf{r}) H^{\prime} \psi_{i}^{(0)}(\mathbf{r}) d^{3} x\right|^{2}
$$

where $\left.p=\sqrt{2 m\left(E_{i}^{(0)}+\hbar \omega\right)}\right)=\sqrt{2 m\left(\hbar \omega-E_{B}\right)}$. For sinisoidal fields

$$
\sigma_{P D}=\frac{\hbar \omega}{\frac{1}{2} \epsilon_{0} c \omega^{2} A_{0}^{2}} \Gamma_{P D}
$$

This gives the differential cross-section as

$$
\frac{d \sigma_{P D}}{d \Omega}=\frac{2 \hbar \omega}{\epsilon_{0} c \omega^{2} A_{0}^{2}} \frac{d \Gamma_{P D}}{d \Omega}=\frac{2 \mathcal{V} m}{(2 \pi)^{2} \hbar^{3} \epsilon_{0} c A_{0}^{2}} \frac{p}{\omega}\left|\int \psi_{f}^{(0) *}(\mathbf{r}) H^{\prime} \psi_{i}^{(0)}(\mathbf{r}) d^{3} x\right|^{2}
$$

\subsection{Application to Two Particle Systems}

Recall that the TDSE of the two particle system with the dipole approximation is

$$
i \hbar \frac{\partial}{\partial t} \Psi\left(\mathbf{r}_{1}, \mathbf{r}_{2}, t\right)=\left[H_{0}+\frac{e}{m} \mathbf{A}(t) \cdot \mathbf{P}\right] \Psi\left(\mathbf{r}_{1}, \mathbf{r}_{2}, t\right)
$$

where we have not included the second order term $\frac{e^{2}}{m} \mathbf{A}^{2}(t)$. All second order terms are zero in first order perturbation theory. Therefore, the perturbation term will be

$$
\begin{aligned}
H^{\prime}(t) & =\quad \frac{e}{m} \mathbf{A}(t) \cdot \mathbf{P} \\
& =\frac{e}{m} A_{0} \frac{1}{2}\left(e^{-i \omega t}+e^{i \omega t}\right) \hat{\boldsymbol{\epsilon}} \cdot \mathbf{P} \\
& =\quad \frac{e}{m} A_{0} \frac{1}{2} e^{-i \omega t} \hat{\boldsymbol{\epsilon}} \cdot \mathbf{P},
\end{aligned}
$$

where only the first exponential will cause a transition from a lower energy state to a higher one (photodetachment). If we had used a second quantized electromagnetic field, the annihilation operator would have killed the other state. The unperturbed 
Hamiltonian will be

$$
H_{0}=\frac{\mathbf{p}_{1}^{2}}{2 m}+\frac{\mathbf{p}_{2}^{2}}{2 m}-\frac{Z e^{2}}{\left(4 \pi \epsilon_{0}\right) r_{1}}-\frac{Z e^{2}}{\left(4 \pi \epsilon_{0}\right) r_{2}}+\frac{e^{2}}{\left(4 \pi \epsilon_{0}\right) r_{12}}
$$

Solutions to the unperturbed Hamiltonian are simply the wavefunctions $\psi_{n}^{(0)}\left(\mathbf{r}_{1}, \mathbf{r}_{2}\right)$ and can be found by time-independent perturbation theory, the variational principle, or other approximation methods. Keating, Pak and Straton have also done analysis using highly accurate variational wavefunctions [25]. Thus,

$$
\begin{aligned}
\left|\int \psi_{f}^{(0) *} H^{\prime} \psi_{i}^{(0)} d^{3} x\right|^{2} & =\left|\iint \psi_{f}^{(0) *}\left(\mathbf{r}_{1}, \mathbf{r}_{2}\right)\left(\frac{e A_{0}}{2 m} \hat{\boldsymbol{\epsilon}} \cdot \mathbf{P}\right) \psi_{i}^{(0)}\left(\mathbf{r}_{1}, \mathbf{r}_{2}\right) d^{3} x_{1} d^{3} x_{2}\right|^{2} \\
& =\frac{e^{2} A_{0}^{2}}{4 m^{2}}\left|\iint \psi_{f}^{(0) *}\left(\mathbf{r}_{1}, \mathbf{r}_{2}\right)(\hat{\boldsymbol{\epsilon}} \cdot \mathbf{P}) \psi_{i}^{(0)}\left(\mathbf{r}_{1}, \mathbf{r}_{2}\right) d^{3} x_{1} d^{3} x_{2}\right|^{2}
\end{aligned}
$$

where the periodic time dependence of the perturbation was already taken care of by Fermi's Golden Rule.

We will use the so called effective range theory, where the term $\frac{Z e^{2}}{\left(4 \pi \epsilon_{0}\right) r_{2}}+\frac{e^{2}}{\left(4 \pi \epsilon_{0}\right) r_{12}}$ is considered roughly a constant $e V_{\text {eff }}$ over some small effective range $a$ and zero outside it for the (outer) second electron. The second electron is effectively in a finite spherical well. With the effective range approximation, the two electron wavefunctions become separable. This alleviates a fair amount of complexity from our further analysis. For details on effective range theory see Appendix C. 


\section{Chapter 5}

\section{The Negative Hydrogen Ion}

\subsection{Photodetachment (PD)}

In this section, we are concerned mainly with Photodetachment (PD) of two particle systems.

$$
H^{-}\left(1 s^{2}{ }^{1} S^{e}\right)+\hbar \omega \rightarrow H(1 s)+e^{-}
$$

The final state will be a free particle and bound particle (total spin $s=0, l=1$, $\left.m_{s}=0\right)[45]$ represented by,

$\psi_{f}^{(0)}\left(\mathbf{r}_{1}, \mathbf{r}_{2}\right)=\psi_{s=0, l=1, m_{s}=0}\left(\mathbf{r}_{1}, \mathbf{r}_{2}\right)=\frac{1}{\sqrt{2}}\left[\psi_{\text {planewave }}\left(\mathbf{r}_{1}\right) \psi_{1 s}\left(\mathbf{r}_{2}\right)+\psi_{\text {planewave }}\left(\mathbf{r}_{2}\right) \psi_{1 s}\left(\mathbf{r}_{1}\right)\right]$

and the initial state will be the ground state of the two particle wavefunction (total $\left.\operatorname{spin} s=0, l=0, m_{s}=0\right)$,

$$
\psi_{i}^{(0)}\left(\mathbf{r}_{1}, \mathbf{r}_{2}\right)=\psi_{s=0, l=0, m_{s}=0}\left(\mathbf{r}_{1}, \mathbf{r}_{2}\right)=\frac{1}{\sqrt{2}}\left[\psi_{e f f}\left(\mathbf{r}_{1}\right) \psi_{1 s}\left(\mathbf{r}_{2}\right)+\psi_{e f f}\left(\mathbf{r}_{2}\right) \psi_{1 s}\left(\mathbf{r}_{1}\right)\right]
$$

where

$$
\psi_{e f f}(\mathbf{r})=\frac{C}{r} \exp (-\gamma r)
$$

is the effective range (or rather zero range!) wavefunction of Bethe [44] and Ohmura and Ohmura (Yes, both authors are Ohmura) [46, 47]. For details on effective range 


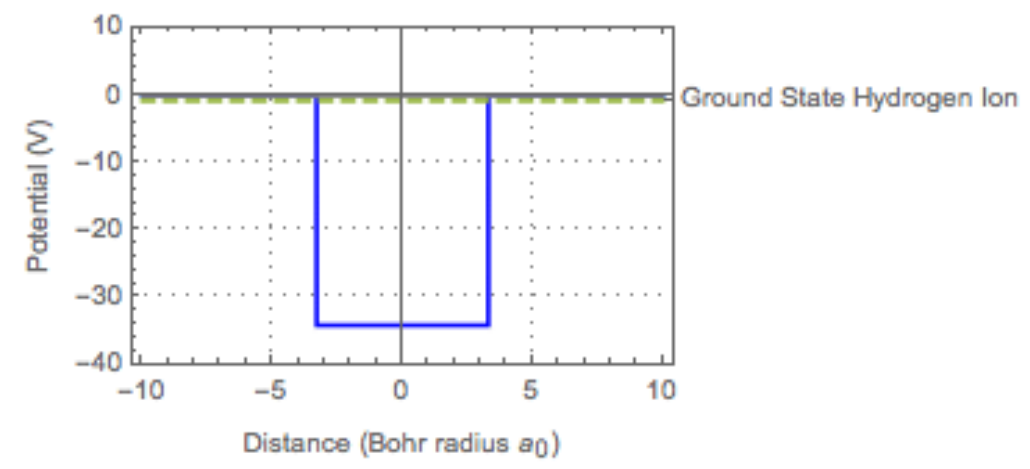

Figure 5.1: Effective Range Potential: The potential felt by the second electron is approximated by a finite square well. The $\mathrm{x}$-axis is the distance in the linear coordinate $x$, not the radius.

theory see Appendix C. (The photon in the initial state will carry $l=1$ angular momentum. We will not be explicit about the spinner or photon components of the states, although one should keep them in mind when thinking about conservation of energy and momentum.)

In fact, the negative hydrogen ion has only one bound state. The bound state energy for the negative hydrogen ion without higher order corrections of mass polarization and relativistic corrections is $E_{B}=0.7551 \mathrm{eV}=1.210 \times 10^{-19} \mathrm{~J}=0.02775$ Hartree, $\gamma=\sqrt{2 m E_{B}} / \hbar=4.452 \times 10^{9} \mathrm{~m}^{-1}=0.2356 a_{0}^{-1}$ or $1 / \gamma=4.245 a_{0}$, where $E_{B}=-E_{i}^{(0)}$. Bethe normalizes the wavefunction over all space, giving $C=\sqrt{\gamma / 2 \pi}$. However, we know that Bethe's effective range wavefunction behavior close to the origin is poor. In fact, the wavefunction overestimates (a lot) the probability near the origin and underestimates (a little) the probability far from the origin. To remedy the effective range wavefunction Bethe later defined

$$
\psi_{e f f}(\mathbf{r})=\left\{\begin{array}{cc}
\frac{A}{r} \sin \left(k^{\prime} r\right) & r<a \\
\frac{C}{r} \exp (-\gamma r) & r>a
\end{array}\right.
$$

where $a$ is the effective range, and adjusted the value of $C$ up somewhat from $\sqrt{\gamma / 2 \pi}$ 
to get a more accurate representation of the wavefunction far from the origin.

The wavefunction and its derivative should be continuous at $r=0$, leading to the choice of $\sin \left(k^{\prime} r\right)$ near the origin. The wavefunction and its derivative should also be continuous at $r=a$. This condition gives

$$
\cot \left(k^{\prime} a\right)=-\frac{\gamma}{k^{\prime}}=-\frac{\gamma a}{k^{\prime} a},
$$

which conveniently does not involve $A$ and $C$ and given $\gamma=\frac{\sqrt{2 m E_{B}}}{\hbar}$ and $a$ sets $k^{\prime}$. This in turn can be used to determine $V_{\text {eff }}$, the depth of the spherical well,

$$
e V_{e f f}=\frac{\hbar^{2} k^{\prime 2}}{2 m}+E_{B} \approx 34 e V
$$

For atomic systems the effective range is on the order of the Bohr radius; for the hydrogen ion $a \approx 3.3 a_{0}$. These values set $k^{\prime} a \gtrsim \pi / 2$ or more precisely

$$
k^{\prime} a=1.95 .
$$

Given $\gamma$ and $a$, the normalization sets the value of $C$, to

$$
C=\frac{\sqrt{\gamma / 2 \pi} e^{\gamma a}}{\sqrt{1+\frac{\gamma a}{\sin ^{2}\left(k^{\prime} a\right)}\left(1-\frac{\sin \left(2 k^{\prime} a\right)}{2 k^{\prime} a}\right)}}
$$

which has the approximations in literature of $C \approx \sqrt{\gamma / 2 \pi}\left(1+\frac{1}{2} \gamma a+\frac{3}{8}(\gamma a)^{2}\right)$ and the less accurate $C \approx \sqrt{\gamma / 2 \pi}(1-\gamma a)^{-1 / 2}$.

Yet another effective wavefunction that has been used is:

$$
\psi_{\text {eff }}(\mathbf{r})=\left\{\begin{array}{cc}
0 & r<a \\
\frac{C}{r} \exp (-\gamma r) & r>a
\end{array},\right.
$$


where normalization constant is $C=\sqrt{\gamma / 2 \pi} e^{\gamma a}$. Suffice to say, the normalization constant $C$ is of theoretical interest and finding an accurate value is important in finding good agreement with experiment. Determining the value of $C$ is now equivalent to finding the value of $a$. We find

$$
C=4.343 \times 10^{4} m^{-1 / 2}=0.3159 a_{0}^{-1 / 2},
$$

where $m$ is meters and $a_{0}$ is the bohr radius. In practice, variational calculations are used to find highly accurate ground state energies and wavefunctions. In fact, the values of $C$ are usually inferred from the highly accurate (and more complicated) variational wavefunctions (see $[46,47,48,49,50]$ ) and the effective range is then determined. From the variational calculations, one can easily "fit" the much simpler effective range wavefunction to highly accurate values for $C$. At this point, it becomes useful to define a constant that is related to $C$ and is unitless, namely

$$
\mathcal{C}=\frac{C}{\sqrt{\gamma / 2 \pi}}=1.631
$$

which is the correction factor from Bethe's first (not so great) approximation.

Having defined the wavefunctions for the system, we now look at perturbation amplitude. There are many forms found in literature. One commonly used form within the dipole approximation is the so called length form. Using the fact that $\mathbf{P}=\frac{m}{i \hbar}\left[\mathbf{R}, H_{0}\right]$,

$$
\begin{aligned}
\left|\int \psi_{f}^{(0) *}(\mathbf{r}) H^{\prime} \psi_{i}^{(0)}(\mathbf{r}) d^{3} x\right|^{2} & =\quad\left|\iint \psi_{f}^{(0) *}\left(\mathbf{r}_{1}, \mathbf{r}_{2}\right)\left(\frac{e A_{0}}{2 m} \hat{\epsilon} \cdot \mathbf{P}\right) \psi_{i}^{(0)}\left(\mathbf{r}_{1}, \mathbf{r}_{2}\right) d^{3} x_{1} d^{3} x_{2}\right|^{2} \\
& =\quad \frac{e^{2} A_{0}^{2}}{4 m^{2}}\left|\iint \psi_{f}^{(0) *}\left(\mathbf{r}_{1}, \mathbf{r}_{2}\right)\left(\hat{\epsilon} \cdot \frac{m}{i \hbar}\left[\mathbf{R}, H_{0}\right]\right) \psi_{i}^{(0)}\left(\mathbf{r}_{1}, \mathbf{r}_{2}\right) d^{3} x_{1} d^{3} x_{2}\right|^{2} \\
& =\frac{e^{2} A_{0}^{2} \frac{m^{2}}{4 m^{2}} \hbar^{2}}{\hbar^{2}}\left(E_{f}^{(0)}-E_{i}^{(0)}\right)^{2}\left|\iint \psi_{f}^{(0) *}\left(\mathbf{r}_{1}, \mathbf{r}_{2}\right)(\hat{\epsilon} \cdot \mathbf{R}) \psi_{i}^{(0)}\left(\mathbf{r}_{1}, \mathbf{r}_{2}\right) d^{3} x_{1} d^{3} x_{2}\right|^{2}
\end{aligned}
$$


We note that the above procedure is only applicable within the dipole approximation. Using $\mathbf{D}=-e \mathbf{R}$, we find

$$
\frac{d \sigma_{P D}}{d \Omega}=\frac{\mathcal{V} m}{2(2 \pi)^{2} \hbar^{3} \epsilon_{0} c} p \omega\left|\iint \psi_{f}^{(0) *}\left(\mathbf{r}_{1}, \mathbf{r}_{2}\right)(\hat{\boldsymbol{\epsilon}} \cdot \mathbf{D}) \psi_{i}^{(0)}\left(\mathbf{r}_{1}, \mathbf{r}_{2}\right) d^{3} x_{1} d^{3} x_{2}\right|^{2}
$$

or

$$
\frac{d \sigma_{P D}}{d \Omega}=\alpha \frac{(2 \pi)^{2} \mathcal{V} m^{2}}{h^{2} e^{2}} f v\left|\iint \psi_{f}^{(0) *}\left(\mathbf{r}_{1}, \mathbf{r}_{2}\right)(\hat{\boldsymbol{\epsilon}} \cdot \mathbf{D}) \psi_{i}^{(0)}\left(\mathbf{r}_{1}, \mathbf{r}_{2}\right) d^{3} x_{1} d^{3} x_{2}\right|^{2}
$$

is also a commonly seen form, where $f$ is the frequency of the laser field.

Going back to the so called velocity form, we find

$$
\left|\int \psi_{f}^{(0) *} H^{\prime} \psi_{i}^{(0)} d^{3} x\right|^{2}=\frac{e^{2} A_{0}^{2}}{4 m^{2}}\left|\int \frac{1}{\sqrt{\mathcal{V}}} \exp (-i \mathbf{k} \cdot \mathbf{r})(\hat{\boldsymbol{\epsilon}} \cdot \mathbf{p}) \frac{C}{r} \exp (-\gamma r) d^{3} x\right|^{2}
$$

Using integration by parts (or the fact that $\mathbf{p}$ is Hermitian), we find the photodetachment differential cross-section to be

$$
\begin{array}{rlrl}
\frac{d \sigma_{P D}}{d \Omega} & =\frac{\alpha}{2 \pi} \frac{\mathcal{V}}{\hbar^{2} m} \frac{p}{\omega}\left|\int \frac{1}{\sqrt{\mathcal{V}}} \exp (-i \mathbf{k} \cdot \mathbf{r})(\hat{\boldsymbol{\epsilon}} \cdot \mathbf{p}) \psi_{\text {eff }}(\mathbf{r}) d^{3} x\right|^{2} \\
& =\frac{\alpha}{2 \pi} \frac{\mathcal{V}}{m} \frac{p}{\omega}\left|\int\left(\hat{\boldsymbol{\epsilon}} \cdot \boldsymbol{\nabla} \frac{1}{\sqrt{\mathcal{V}}} \exp (-i \mathbf{k} \cdot \mathbf{r})\right) \psi_{\text {eff }}(\mathbf{r}) d^{3} x\right|^{2} \\
& = & \frac{\alpha}{2 \pi} \frac{\mathcal{V}}{\hbar^{2} m} \frac{p}{\omega}|\hat{\boldsymbol{\epsilon}} \cdot \mathbf{p}|^{2}\left|\widetilde{\psi}_{\text {eff }}(\mathbf{k})\right|^{2}
\end{array}
$$

where $\widetilde{\psi}_{i o n}(\mathbf{k})$ is the Fourier transfrom of $\psi_{\text {eff }}(\mathbf{r})$,

$$
\widetilde{\psi}_{e f f}(\mathbf{k})=\frac{1}{\sqrt{\mathcal{V}}} \int \exp (-i \mathbf{k} \cdot \mathbf{r}) \psi_{e f f}(\mathbf{r}) d^{3} x
$$

This is the starting point of Keating's paper [24]. Notice the cross section is not a function of the amplitude of the field $A_{0}$. The $A_{0}^{2}$ terms in both the numerator and denominator canceled each other out. In first order perturbation theory, the 


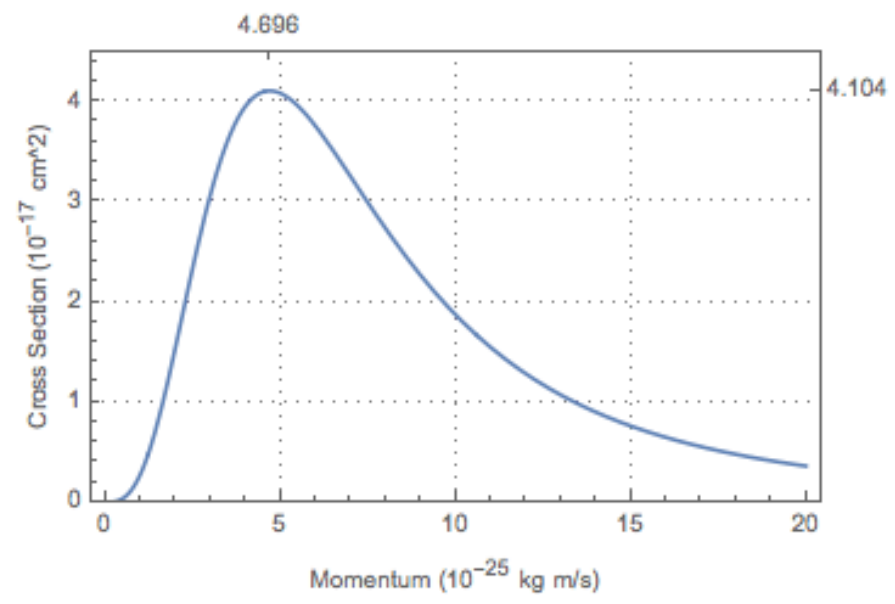

Figure 5.2: Cross Section vs. Momentum for Photodetachment (PD)

calculation of cross-section will have the amplitude of the field cancel in this way. However, we know that actual cross-section is a function of field strength; therefore, we will need to look for methods that go beyond perturbation theory.

The integral in the last equation is a simple Fourier transform. Thus,

$$
\begin{aligned}
& \widetilde{\psi}_{e f f}(\mathbf{k})=\frac{1}{\sqrt{\nu}} \int \exp (-i \mathbf{k} \cdot \mathbf{r}) \frac{C}{r} \exp (-\gamma r) d^{3} x \\
& =\quad \frac{1}{\sqrt{v}} \frac{4 \pi C}{k^{2}+\gamma^{2}},
\end{aligned}
$$

giving us a differential cross section,

$$
\frac{d \sigma_{P D}}{d \Omega}=\frac{\alpha}{2 \pi} \frac{C^{2}}{\hbar^{2} m} \frac{p}{\omega}|\hat{\boldsymbol{\epsilon}} \cdot \mathbf{p}|^{2} \frac{16 \pi^{2}}{\left(k^{2}+\gamma^{2}\right)^{2}} .
$$

Noting that $\int|\hat{\boldsymbol{\epsilon}} \cdot \mathbf{p}|^{2} d \Omega=\frac{4 \pi}{3} p^{2}$. The total cross-section written in atomic units is

$$
\sigma_{P D}(k)=\alpha \frac{32 \pi}{3} \mathcal{C}^{2} a_{0}^{2}\left(\gamma a_{0}\right) \frac{\left(k a_{0}\right)^{3}}{\left(\left(k a_{0}\right)^{2}+\left(\gamma a_{0}\right)^{2}\right)^{3}}
$$

or more simply 


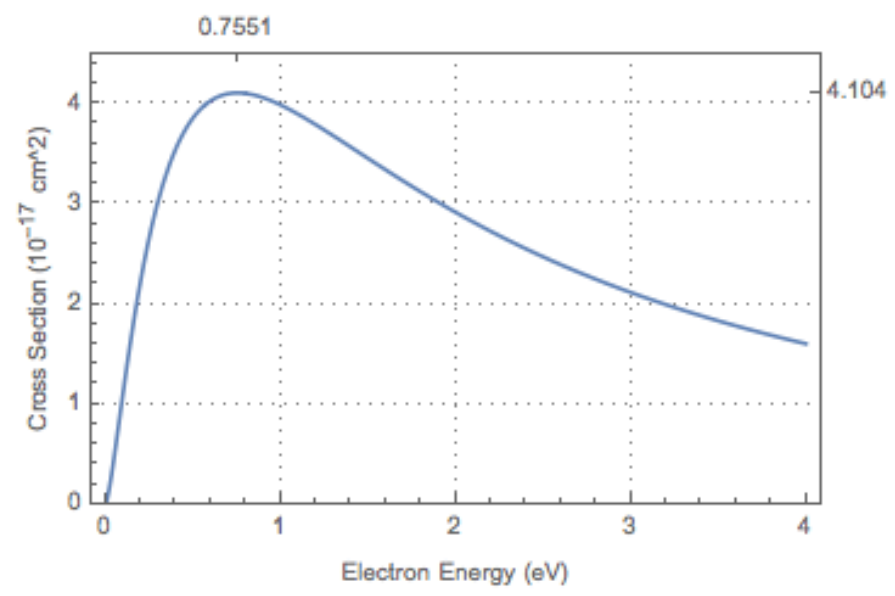

Figure 5.3: Cross Section vs. Electron Energy for Photodetachment (PD)

$$
\sigma_{P D}(k)=4.293 \times 10^{-18} \mathrm{~cm}^{2} \frac{\left(k a_{0}\right)^{3}}{\left(\left(k a_{0}\right)^{2}+\left(\gamma a_{0}\right)^{2}\right)^{3}} .
$$

The total cross-section as a function of electron momentum and in terms of $p_{0}=$ $\hbar \gamma=4.695 \times 10^{-25} \mathrm{~kg} \frac{\mathrm{m}}{\mathrm{s}}=0.2356 \mathrm{au}\left(v_{0}=5.154 \times 10^{5} \mathrm{~m} / \mathrm{s}\right)$ is

$$
\sigma_{P D}(p)=3.283 \times 10^{-16} \mathrm{~cm}^{2} \frac{\left(\frac{p}{p_{0}}\right)^{3}}{\left(\left(\frac{p}{p_{0}}\right)^{2}+1\right)^{3}},
$$

which is a maximum at $p=p_{0}$, giving us the maximum cross-section to be

$$
\sigma_{P D}\left(p_{0}\right)=4.104 \times 10^{-17} \mathrm{~cm}^{2} .
$$

The maximum rate coefficient is therefore

$$
\alpha_{P D}(\max )=c \sigma_{P D}(\max )=1.230 \times 10^{-6} \frac{\mathrm{cm}^{3}}{\mathrm{~s}} .
$$

and rms rate coefficient is

$$
\alpha_{P D}\left(p_{r m s}\right)=c \sigma_{P D}\left(p_{r m s}\right)=6.940 \times 10^{-10} \frac{\mathrm{cm}^{3}}{\mathrm{~s}} .
$$




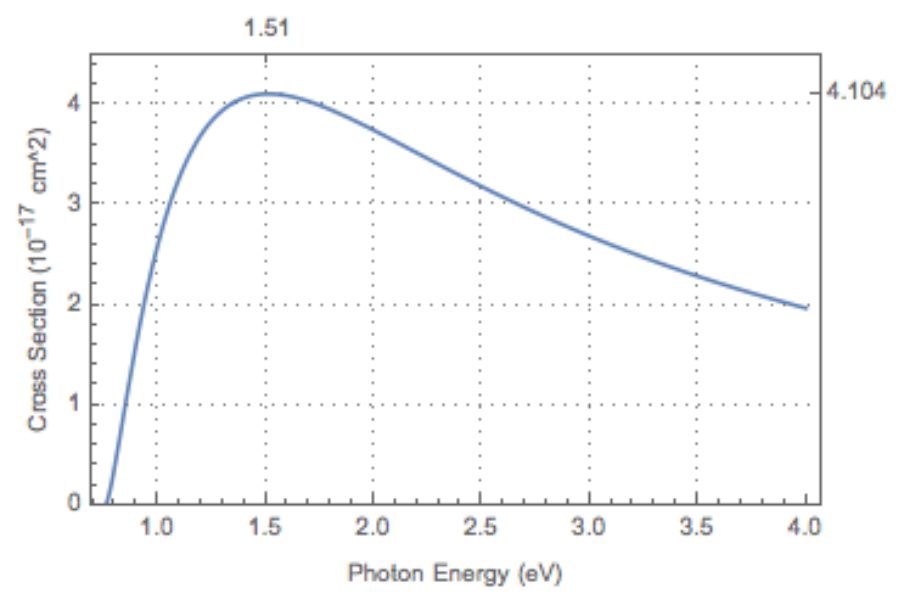

Figure 5.4: Cross Section vs. Photon Energy for Photodetachment (PD)

If the experiment detects the ejected electrons and measures their energy, we have the total cross-section as a function of electron energy. This would be

$$
\sigma_{P D}\left(E_{k}\right)=3.283 \times 10^{-16} \mathrm{~cm}^{2} \frac{\left(\frac{E_{k}}{E_{B}}\right)^{3 / 2}}{\left(\frac{E_{k}}{E_{B}}+1\right)^{3}} .
$$

In experiments, we usually don't have direct control over the momentum of the ejected electrons. Rather, we control the incoming photon energy, that is to say the wavelength of the light. Using the fact, $\hbar \omega=\frac{p^{2}}{2 m}+E_{B}$, we find the total cross-section as a function of photon energy to be

$$
\sigma_{P D}(\hbar \omega)=3.283 \times 10^{-16} \mathrm{~cm}^{2} \frac{\left(\frac{\hbar \omega}{E_{B}}-1\right)^{3 / 2}}{\left(\frac{\hbar \omega}{E_{B}}\right)^{3}}
$$

which is maximum at $\hbar \omega=2 E_{B}$.

This implies the wavelength at which maximum photo-detachment is

$$
\lambda_{\max }=\frac{h c}{2 E_{B}}=821 \mathrm{~nm}
$$




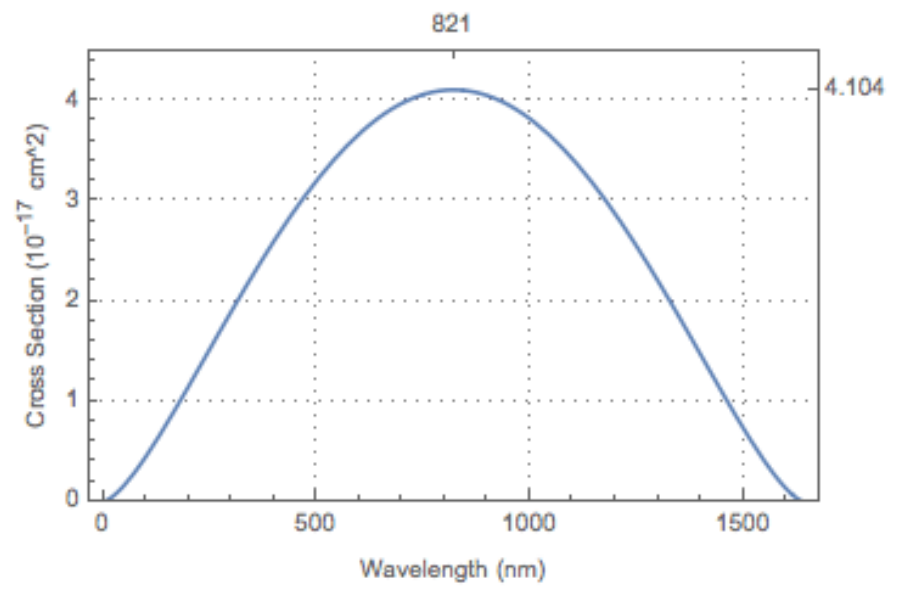

Figure 5.5: Cross Section vs. Wavelength for Photodetachment (PD). For comparison and agreement with experiments see Keating [24, 25].

where $h=6.626 \times 10^{-34} \frac{\mathrm{kg} \cdot \mathrm{m}^{2}}{\mathrm{~s}}$ is Planck's constant, $c=2.998 \times 10^{8} \frac{\mathrm{m}}{\mathrm{s}}$ is the speed of light and $\lambda$ is the wavelength. We can write the total cross-section as a function of wavelength, we find

$$
\sigma_{P D}(\lambda)=3.283 \times 10^{-16} \mathrm{~cm}^{2}\left(\frac{\lambda}{\lambda_{0}}\right)^{3 / 2}\left(1-\frac{\lambda}{\lambda_{0}}\right)^{3 / 2}
$$

where $\lambda_{0}=h c / E_{B}=1642 \mathrm{~nm}$. Once we have the total cross-section, one can easily go back to total rates by

$$
\Gamma_{P D}=\frac{I}{\hbar \omega} \sigma_{P D}
$$

where we see that the total rate is linear in laser intensity.

\subsection{Spontaneous Radiative Attachment (RA)}

In this section, we are concerned mainly with Spontaneous Radiative Attachment (RA) of two particle systems.

$$
H(1 s)+e^{-} \rightarrow H^{-}\left(1 s^{2}{ }^{1} S^{e}\right)+\hbar \omega .
$$


Since there is no applied field with Spontaneous Radiative Attachment (RA) of electrons, it is not easily explained within the framework of the Schrödinger equation. The cleanest way of getting spontaneous emission of a photon (attachment of electron) is by means of second quantization. However, Einstein was the first person able to deduce spontaneous emission. He did so not through second quantization, which was not yet known, but through the use of the rate equation and the Planck distribution. Recall the rate equation (with spontaneous emission!) is

$$
\frac{d n_{H^{-}}}{d t}=\beta_{S A} n n_{e} n_{H}-\alpha_{P D} n n_{H^{-}}+\alpha_{R A} n_{e} n_{H} .
$$

At equilibrium we have

$$
\alpha_{P D} n n_{H^{-}}-\beta_{S A} n n_{e} n_{H}=\alpha_{R A} n_{e} n_{H} .
$$

Solving for the photon number density

$$
n=\frac{\alpha_{R A} n_{e}}{\alpha_{P D}\left(\frac{n_{H}-}{n_{H}}\right)-\beta_{S A} n_{e}} .
$$

We know from statistical mechanics the number of particles with energy $\hbar \omega$, in thermal equilibrium at temperature $T$, is proportional to the Boltzmann factor, $\exp \left(\hbar \omega / k_{B} T\right)$. Therefore,

$$
\frac{\mathcal{N}_{H^{-}}}{\mathcal{N}_{H}}=e^{\frac{\hbar \omega}{k_{B} T}}
$$

Plugging this into the previous equation, we find

$$
n=\frac{n_{e}}{\frac{\alpha_{P D}}{\alpha_{R A}} e^{\frac{\hbar \omega}{k_{B} T}}-\frac{\beta_{S A} n_{e}}{\alpha_{R A}}} .
$$


At this point, Einstein knew the Planck distribution was

$$
\frac{\mathcal{N}}{\mathcal{N}_{e}}=\frac{1}{e^{\frac{\hbar \omega}{k_{B} T}}-1} .
$$

By comparing the last two equations we conclude that at equilibrium,

$$
\alpha_{P D}=\alpha_{R A}
$$

and

$$
\beta_{S A} n_{e}=\alpha_{R A} .
$$

Combining these two equations,

$$
\beta_{S A} n_{e}=\alpha_{P D},
$$

which is again a form of the principle of detailed balancing. Had we not included spontaneous emission in the rate equation, then we would not be able to get to the Planck distribution. In essence, Einstein was forced to add spontaneous emission into the rate equation in order for Planck to have the correct distribution. Since the Planck distribution was backed up by experiment, Einstein had good reason to believe it correct. By adding spontaneous emission, Einstein made the two theories consistent.

Now we will use the principle of detailed balancing to obtain the cross-section for spontaneous radiative attachment of a second electron. Starting with the principle of detailed balancing,

$$
\sigma_{R A}=\frac{1}{2} \frac{\left(\frac{\hbar \omega}{c}\right)^{2}}{p^{2}} \sigma_{P D}
$$



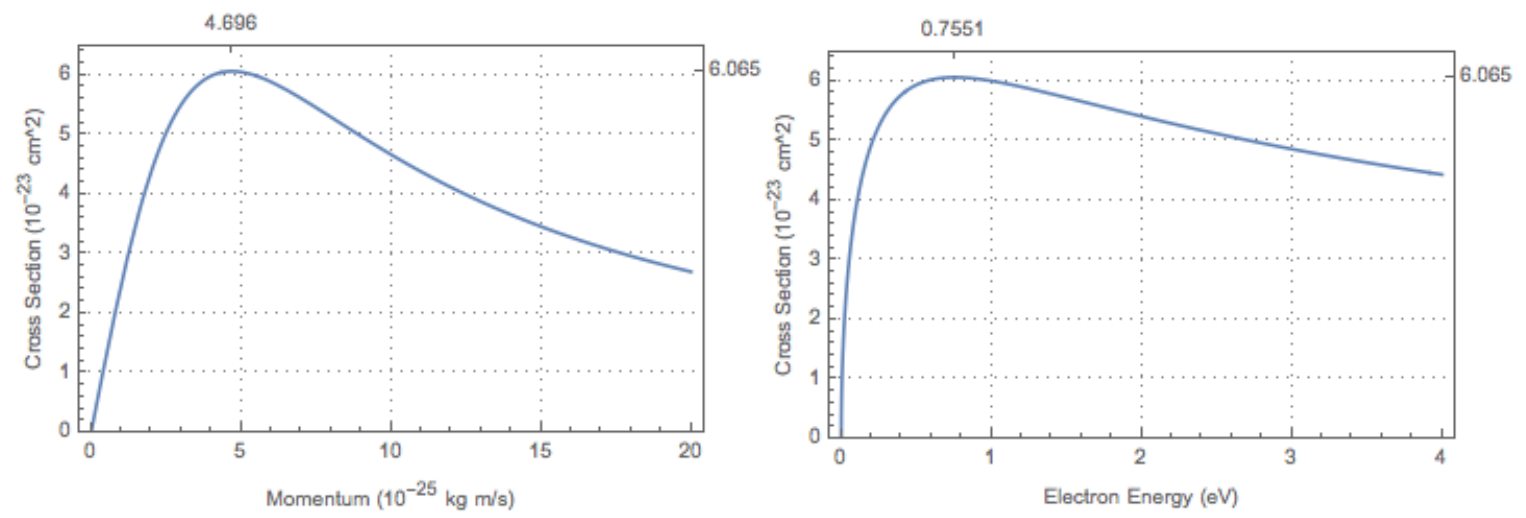

Figure 5.6: Cross Sections for Spontaneous Radiative Attachment (RA): Left - vs. Momentum. Right - vs. Electron Energy.

we find the cross-section as a function of momentum to be

$$
\sigma_{R A}(p)=1.213 \times 10^{-22} \mathrm{~cm}^{2} \frac{\left(\frac{p}{p_{0}}\right)}{\left(\frac{p}{p_{0}}\right)^{2}+1}
$$

which is a maximum at $p=p_{0}$, where $\sigma_{R A}\left(p_{0}\right)=6.065 \times 10^{-23} \mathrm{~cm}^{2}$. As a function of electron energy the cross-section is

$$
\sigma_{R A}\left(E_{k}\right)=1.213 \times 10^{-22} \mathrm{~cm}^{2} \frac{\left(\frac{E_{k}}{E_{B}}\right)^{1 / 2}}{\left(\frac{E_{k}}{E_{B}}\right)+1} .
$$

In experiments, the incoming electrons with a known momentum and flux are made to collide with target atoms such that the rate can be given by

$$
\Gamma_{R A}=\frac{\mathcal{N}_{e}}{\mathcal{V}} \alpha_{R A}=\frac{\mathcal{N}_{e}}{\mathcal{V}} v \sigma_{R A},
$$

where $v$ is the velocity of the electrons and $\alpha_{R A}(v)=v \sigma_{R A}$ (not to be confused with $\alpha$, the fine structure constant) is called the attachment rate coefficient or constant. The rate coefficient is defined in such a way as to get all velocity dependence into one 

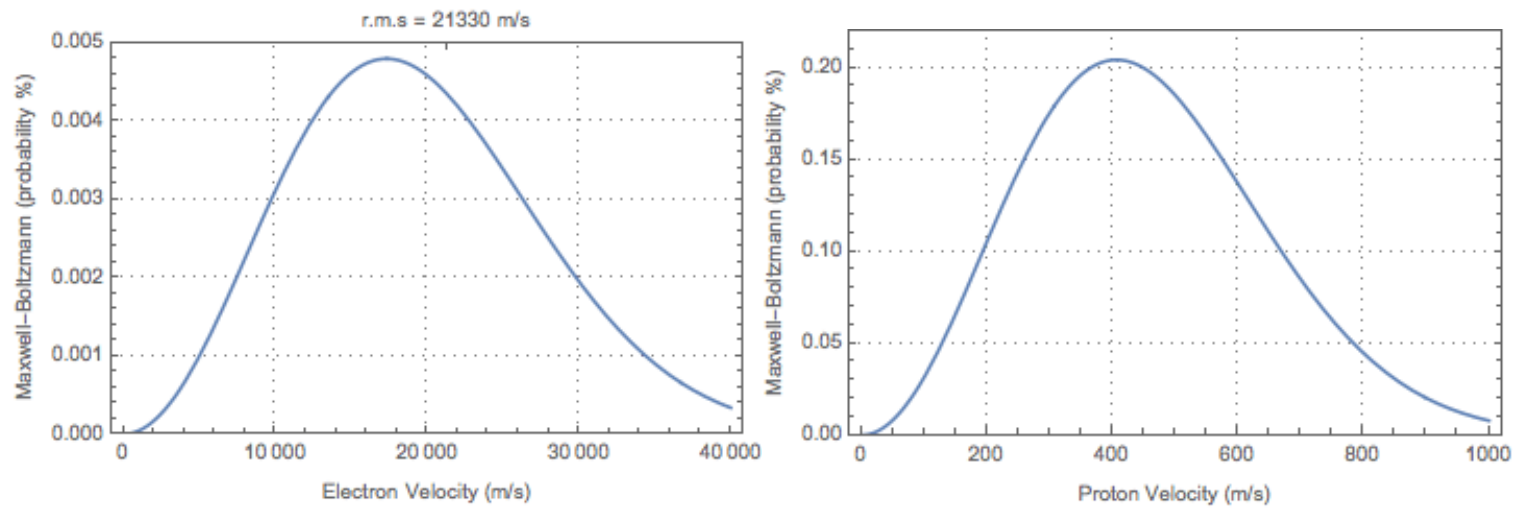

Figure 5.7: Maxwell-Boltzmann Distributions: Left - Electron. Right - Proton. The Temperature is $T=10 \mathrm{~K}$.

coefficient. If all the electrons were at the velocity $v_{0}=5.154 \times 10^{5} \mathrm{~m} / \mathrm{s}$, then

$$
\begin{gathered}
\alpha_{R A}(\max )=v_{0} \sigma_{R A}(\max )= \\
\left(5.154 \times 10^{7} \mathrm{~cm} / \mathrm{s}\right)\left(6.065 \times 10^{-23} \mathrm{~cm}^{2}\right)=3.123 \times 10^{-15} \frac{\mathrm{cm}^{3}}{\mathrm{~s}} .
\end{gathered}
$$

However, in reality the flux of electrons has a distribution of velocities. This distribrution of velocities is best characterized by the temperature of the electron gas. Our electron gas happens to have a temperature of about $10 \mathrm{~K}$. The distribution of electron velocities is described by the Maxwell-Boltzmann distribution

$$
f(v)=\left(\frac{m}{2 \pi k_{B} T}\right)^{3 / 2} 4 \pi v^{2} e^{-\frac{m v^{2}}{2 k_{B} T}},
$$

or as a function of electron energy

$$
f\left(E_{k}\right)=\left(\frac{m}{2 \pi k_{B} T}\right)^{3 / 2} 4 \pi \frac{2}{m} E_{k} e^{-\frac{E_{k}}{k_{B} T}},
$$

where $k_{B}=8.617 \times 10^{-5} \mathrm{eV} / \mathrm{K}=1.381 \times 10^{-23} \mathrm{~J} / \mathrm{K}$ is the Boltzmann's constant, $T$ is temperature and $f(v)$ is normalized such that $\int f(v) d v=1$. The temperature 
dependent rate coefficient is then

$$
\alpha_{R A}(T)=\int_{0}^{\infty} \alpha_{R A}(v) f(v) d v=\int_{0}^{\infty} v \sigma_{R A}(v) f(v) d v
$$

or in terms of electron energy

$$
\alpha_{R A}(T)=\frac{1}{m} \int_{0}^{\infty} \sigma_{R A}\left(E_{k}\right) f\left(E_{k}\right) d E_{k}
$$

Plugging in the cross-section and Maxwell-Boltzmann distribution, we get

$$
\alpha_{R A}(T)=1.213 \times 10^{-22} \mathrm{~cm}^{2}\left(\frac{m}{2 \pi k_{B} T}\right)^{3 / 2} \frac{8 \pi E_{B}}{m^{2}} \int_{0}^{\infty} \frac{\left(\frac{E_{k}}{E_{B}}\right)^{3 / 2}}{\left(\frac{E_{k}}{E_{B}}\right)+1} e^{-\frac{E_{k}}{k_{B} T}} d E_{k}
$$

Changing variables to $x=\frac{E_{k}}{k_{B} T}$ makes this a bit simpler:

$$
\alpha_{R A}(T)=1.213 \times 10^{-22} \mathrm{~cm}^{2}\left(\frac{m}{2 \pi}\right)^{3 / 2} \frac{8 \pi}{m^{2}} \sqrt{E_{B}} \int_{0}^{\infty} \frac{x^{3 / 2}}{x+\frac{E_{B}}{k_{B} T}} e^{-x} d x
$$

The integral can be found in a table of integrals [51] (GR 3.383.10).

$$
\int_{0}^{\infty} \frac{x^{3 / 2}}{x+\mu} e^{-x} d x=\mu^{3 / 2} e^{\mu} \Gamma\left(\frac{5}{2}\right) \Gamma\left(-\frac{3}{2}, \mu\right)=\frac{3}{4} \sqrt{\pi} \mu^{3 / 2} e^{\mu} \Gamma\left(-\frac{3}{2}, \mu\right)
$$

where $\Gamma(s, c)=\int_{c}^{\infty} y^{s-1} e^{-y} d y$ is the incomplete gamma function (not to be confused with the rate) and $\mu$ is a "constant" that happens to be $\frac{E_{B}}{k_{b} T}$ for us. Thus,

$$
\alpha_{R A}(T)=1.213 \times 10^{-22} \mathrm{~cm}^{2} 3 \sqrt{\frac{E_{B}}{2 m}}\left(\frac{E_{B}}{k_{B} T}\right)^{3 / 2} e^{\frac{E_{B}}{k_{B} T}} \Gamma\left(-\frac{3}{2}, \frac{E_{B}}{k_{B} T}\right) .
$$


At low temperatures, $\mu$ is large. Therefore, we will use

$$
\Gamma\left(-\frac{3}{2}, \mu\right) \approx e^{-\mu} \mu^{-5 / 2} \quad \mu \gg 1 .
$$

Thus, for $T \ll \frac{E_{B}}{k_{B}} \approx 9000 K$, we have

$$
\alpha_{R A}(T) \approx 9.378 \times 10^{-15} \frac{\mathrm{cm}^{3}}{s}\left(\frac{k_{B} T}{E_{B}}\right),
$$

in standard units. In atomic units, we find

$$
\alpha_{R A}(T) \approx 3.379 \times 10^{-13} \frac{\mathrm{cm}^{3}}{s}\left(\frac{k_{B} T}{E_{a . u .}}\right) .
$$

Finally, in "SI like" units

$$
\alpha_{R A}(T) \approx 1.070 \times 10^{-18} \frac{\mathrm{cm}^{3}}{\mathrm{~s} \cdot \mathrm{K}} T,
$$

which we can see is linear in temperature.

It so happens that at our low temperatures, $T=10 \mathrm{~K}$, the distribution of velocities of the electrons is not all that broad. This means most of the electrons have velocities very close to the most probable velocity. Therefore, we could approximate the rate coefficient by considering that most of the electrons are at, or around, the most probable velocity, which happens to be

$$
v_{p}=\sqrt{\frac{2 k_{B} T}{m}}=1.741 \times 10^{4} \mathrm{~m} / \mathrm{s} .
$$

In turn, the most probable momentum is

$$
p_{p}=m v_{p}=1.585 \times 10^{-26} \mathrm{~kg} \frac{\mathrm{m}}{\mathrm{s}} .
$$


Using the most probable momentum, we find the cross section to be

$$
\sigma_{R A}\left(p_{p}\right)=1.213 \times 10^{-22} \mathrm{~cm}^{2} \frac{\left(\frac{p_{p}}{p_{0}}\right)}{\left(\frac{p_{p}}{p_{0}}\right)^{2}+1}=4.090 \times 10^{-24} \mathrm{~cm}^{2} .
$$

This gives a rate coefficient of

$$
\begin{gathered}
\alpha_{R A}\left(p_{p}\right)=v_{p} \sigma_{R A}\left(p_{p}\right)= \\
\left(1.741 \times 10^{6} \mathrm{~cm} / \mathrm{s}\right)\left(4.090 \times 10^{-24} \mathrm{~cm}^{2}\right)=7.121 \times 10^{-18} \frac{\mathrm{cm}^{3}}{\mathrm{~s}} .
\end{gathered}
$$

This approximate value is only about $30 \%$ lower than the exact Maxwell-Boltzmann distribution calculation. Another way of approximating the rate coefficient is the average velocity,

$$
v_{\text {ave }}=\sqrt{\frac{8}{\pi} \frac{k_{B} T}{m}}=1.865 \times 10^{4} \mathrm{~m} / \mathrm{s} .
$$

This gives the average momentum as

$$
p_{\text {ave }}=m v_{\text {ave }}=1.790 \times 10^{-26} \mathrm{~kg} \frac{\mathrm{m}}{\mathrm{s}} .
$$

Using the average momentum, we find the cross section to be

$$
\sigma_{R A}\left(p_{\text {ave }}\right)=1.213 \times 10^{-22} \mathrm{~cm}^{2} \frac{\left(\frac{p_{\text {ave }}}{p_{0}}\right)}{\left(\frac{p_{\text {ave }}}{p_{0}}\right)^{2}+1}=4.617 \times 10^{-24} \mathrm{~cm}^{2}
$$

This gives a rate coefficient of

$$
\alpha_{R A}\left(p_{\text {ave }}\right)=v_{\text {ave }} \sigma_{R A}\left(p_{\text {ave }}\right)=
$$




$$
\left(1.865 \times 10^{6} \mathrm{~cm} / \mathrm{s}\right)\left(4.617 \times 10^{-24} \mathrm{~cm}^{2}\right)=8.612 \times 10^{-18} \frac{\mathrm{cm}^{3}}{\mathrm{~s}} .
$$

This value for the rate coefficient is even more accurate than using the most probable momentum. Finally, the best way to approximate the rate coefficient is the root mean square (rms) velocity,

$$
v_{r m s}=\sqrt{\frac{3 k_{B} T}{m}}=2.133 \times 10^{4} \mathrm{~m} / \mathrm{s},
$$

giving us the rms momentum,

$$
p_{r m s}=m v_{r m s}=1.943 \times 10^{-26} \mathrm{~kg} \frac{\mathrm{m}}{\mathrm{s}}=9.749 \times 10^{-3} \mathrm{a} . u .
$$

Using the rms momentum, we find the cross section to be

$$
\sigma_{R A}\left(p_{r m s}\right)=1.213 \times 10^{-22} \mathrm{~cm}^{2} \frac{\left(\frac{p_{r m s}}{p_{0}}\right)}{\left(\frac{p_{r m s}}{p_{0}}\right)^{2}+1}=5.011 \times 10^{-24} \mathrm{~cm}^{2}
$$

This gives us a rate coefficient of

$$
\begin{gathered}
\alpha_{R A}\left(p_{r m s}\right)=v_{r m s} \sigma_{R A}\left(p_{r m s}\right)= \\
\left(2.133 \times 10^{6} \mathrm{~cm} / \mathrm{s}\right)\left(5.011 \times 10^{-24} \mathrm{~cm}^{2}\right)=1.069 \times 10^{-17} \frac{\mathrm{cm}^{3}}{\mathrm{~s}} .
\end{gathered}
$$

This is not a bad estimate of the rate coefficient! This shows that integrating over the Maxwell-Boltzmann distribution is not necessary for rough approximations of rates. If rough approximations of rates are desired, then simply using the most probable velocity or average velocity is okay and the rms velocity is extremely accurate (at least at temperatures of $T=10 \mathrm{~K}$ ). Plugging the rms velocity into the cross section, we 
find that the rms velocity reproduces the rate coefficient from the Maxwell-Boltzmann distribution perfectly.

$$
\begin{gathered}
\alpha_{R A}(T) \approx \sqrt{\frac{3 k_{B} T}{m}} \sigma_{R A}\left(m \sqrt{\frac{3 k_{B} T}{m}}\right) \approx 1.213 \times 10^{-22} \mathrm{~cm}^{2} v_{0} \frac{\frac{1}{v_{0}^{2}} \frac{3 k_{B} T}{m}}{\frac{1}{v_{0}^{2}} \frac{3 k_{B} T}{m}+1} \\
\approx 6.252 \times 10^{-15} \frac{\mathrm{cm}^{3}}{\mathrm{~s}} \frac{1}{v_{0}^{2}} \frac{3 k_{B} T}{m}=1.070 \times 10^{-18} \frac{\mathrm{cm}^{3}}{\mathrm{~s} \cdot K} T
\end{gathered}
$$

where we have used the approximation $x /(x+1) \approx x$ for small $x$ and $v_{0}=5.154 \times$ $10^{5} \mathrm{~m} / \mathrm{s}$. From now on, using the rms velocity will save us from having to do the extra integration over the Maxwell-Boltzmann distribution.

Finally, we are in the position to estimate the rate of radiative attachment of the second electron. The rate equation is

$$
\frac{d n_{H^{-}}}{d t}=\alpha_{S A} n_{e} n_{H}-\alpha_{P D} n n_{H^{-}}+\alpha_{R A} n_{e} n_{H}
$$

Assuming we do not have a laser field on $\left(\alpha_{S A}=0\right)$, and the reaction starts out with no hydrogen ions, we find the rate to be

$$
\left.\frac{d \mathcal{N}_{H^{-}}}{d t}\right|_{t=0}=\alpha_{R A} n_{e} \mathcal{N}_{H} .
$$

The electron number density of the incoming beam is of the order

$$
n_{e}=\frac{\mathcal{N}_{e}}{\mathcal{V}}=10^{10} \frac{e^{-}}{\mathrm{cm}^{3}} .
$$

A reasonable achievable temperature for the electron beam is about $T=10 \mathrm{~K}$. We could, of course, increase the temperature of the electron gas, thereby increasing the 
rate coefficient. However, this would lead to the escape of our hydrogen ions from their magnetic trap. Using $T=10 \mathrm{~K}$, gives us

$$
\alpha_{R A}(10 K)=10^{-17} \frac{\mathrm{cm}^{3}}{\mathrm{~s}}
$$

Thus, we find the rate of hydrogen ions produced to be

$$
\left.\frac{d \mathcal{N}_{H^{-}}}{d t}\right|_{t=0}=\left(10^{-17} \frac{\mathrm{cm}^{3}}{\mathrm{~s}}\right)\left(10^{10} \frac{\mathrm{e}^{-}}{\mathrm{cm}^{3}}\right)\left(10^{4} \mathrm{H}\right)=10^{-3} \frac{\mathrm{H}^{-}}{\mathrm{s}}
$$

which is low. With even optimistic projections on our trapped antihydrogen yields and active cooling techniques, a rate this low could be difficult to observe. We will therefore look at the stimulated process, which should increase the rates.

\subsection{Stimulated Attachment (SA)}

In this section, we are concerned mainly with Stimulated Attachment (SA) of two particle systems.

$$
H(1 s)+e^{-}+\mathcal{N} \hbar \omega \rightarrow H^{-}\left(1 s^{2}{ }^{1} \stackrel{e}{S}\right)+(\mathcal{N}+1) \hbar \omega
$$

Laser assisted attachment was introduced by Neumann in 1983 [43]. Since there is an external laser field stimulating the process, we can easily set up this system within the framework of the Schrödinger equation. Thus,

$$
\Gamma_{f i}=\frac{2 \pi}{\hbar}\left|\int \psi_{f}^{(0) *}(\mathbf{r}) H^{\prime} \psi_{i}^{(0)}(\mathbf{r}) d^{3} x\right|^{2} \delta\left(E_{f}^{(0)}-E_{i}^{(0)}+\hbar \omega\right)
$$

The detector is usually only sensitive over a small cross section of space and is usually insensitive to the magnitude of the momentum of the ejected photon. Thus, we integrate over all final frequencies of the outgoing photon. Note, we control the 
initial energy of the electron beam and the frequency of the laser. Therefore, the transition rate will be

$$
\begin{gathered}
\Gamma_{S A}=\sum_{f} \Gamma_{f i}=\int \Gamma_{f i} d^{3} n_{f}= \\
\iint\left(\frac{2 \pi}{\hbar}\left|\int \psi_{f}^{(0) *}(\mathbf{r}) H^{\prime} \psi_{i}^{(0)}(\mathbf{r}) d^{3} x\right|^{2} \frac{1}{\hbar} \delta\left(\omega_{f i}+\omega\right)\right) g_{S A} \frac{\mathcal{V}}{(2 \pi)^{3} \hbar^{3}}\left(\frac{\hbar \omega}{c}\right)^{2} \frac{\hbar}{c} d \omega d \Omega
\end{gathered}
$$

We will assume that our detector does not distinguish between polarizations of the ejected photon. The spin correction factor is

$$
\begin{gathered}
g_{R A}=(\text { sum final spin states })(\text { average initial spin states })= \\
\text { polarization (spins states ion }) \frac{1}{\left(2 s_{\text {electron }}+1\right)\left(2 s_{\text {Hydrogen }}+1\right)}=(2)(1) \frac{1}{(2)(2)}=\frac{1}{2},
\end{gathered}
$$

where this time we are only interested in the one spin state of the final ion. Thus, the rate of transition per solid angle is

$$
\frac{d \Gamma_{S A}}{d \Omega}=\frac{\mathcal{V}}{(2 \pi)^{2} \hbar^{4}} \frac{1}{c}\left(\frac{\hbar \omega}{c}\right)^{2}\left|\int \psi_{f}^{(0) *}(\mathbf{r}) H^{\prime} \psi_{i}^{(0)}(\mathbf{r}) d^{3} x\right|^{2}
$$

where this time $\omega=-\left(E_{f}^{(0)}-E_{i}^{(0)}\right) / \hbar=\left(E_{B}+\frac{p^{2}}{2 m}\right) / \hbar$ and we drop the subscripts on the frequency. Using the rms momentum, $p_{r m s}=1.943 \times 10^{-26} \mathrm{~kg} \frac{\mathrm{m}}{\mathrm{s}}$, and $E_{B}=$ $1.210 \times 10^{-19} \mathrm{~J}$, we find the frequency of the laser where stimulated attachment occurs to be

$$
\omega_{S A}=1.149 \times 10^{15} s^{-1}
$$

Since our temperature is low, $p_{r m s}^{2} / 2 m$ is so small in comparsion with $E_{B}$, this value has not strayed too far from $\hbar \omega=E_{B}$. Thus, the wavelength of the laser where stimulated (resonant) attachment occurs is 


$$
\lambda_{S A}=1639 \mathrm{~nm},
$$

which is in the infrared. From th total cross section, we find

$$
\sigma_{S A}=\frac{\Gamma_{S A}}{\Phi_{e}}=\frac{\Gamma_{S A}}{\mathcal{N}_{e} p /(\mathcal{V} m)}=\frac{\hbar \omega}{\frac{1}{2} \epsilon_{0} c \omega^{2} A_{0}^{2}} \frac{m c}{p} \frac{\mathcal{N}}{\mathcal{N}_{e}} \Gamma_{S A},
$$

the differential cross-section to be

$$
\begin{gathered}
\frac{d \sigma_{S A}}{d \Omega}=\frac{\hbar \omega}{\frac{1}{2} \epsilon_{0} c \omega^{2} A_{0}^{2}} \frac{m c}{p} \frac{\mathcal{N}}{\mathcal{N}_{e}} \frac{d \Gamma_{S A}}{d \Omega}= \\
\frac{\hbar \omega}{\frac{1}{2} \epsilon_{0} c \omega^{2} A_{0}^{2}} \frac{m c}{p} \frac{\mathcal{V}}{(2 \pi)^{2} \hbar^{4}} \frac{1}{c} \frac{\mathcal{N}}{\mathcal{N}_{e}}\left(\frac{\hbar \omega}{c}\right)^{2} \frac{1}{2}\left|\int \psi_{f}^{(0) *}(\mathbf{r}) H^{\prime} \psi_{i}^{(0)}(\mathbf{r}) d^{3} x\right|^{2}
\end{gathered}
$$

If we divide the cross-section for stimulated attachment by the cross-section for photodetachment, we find

$$
\frac{\sigma_{S A}}{\sigma_{P D}}=\frac{\mathcal{N}}{\mathcal{N}_{e}} \frac{1}{2} \frac{\left(\frac{\hbar \omega}{c}\right)^{2}}{p^{2}}
$$

which is consistent with the principle of detailed balancing. This could be considered the least hand-waving path to the principle of detailed balancing. Using the principle of detailed balancing we find

$$
\alpha_{S A}=\mathcal{N} \alpha_{R A}=\mathcal{N}\left(1.070 \times 10^{-18} \frac{\mathrm{cm}^{3}}{\mathrm{~s} \cdot K} T\right)
$$

where the number of photons for a reasonable laser intensity that is well within the limits of perturbation theory, $I=10^{10} \frac{\mathrm{W}}{\mathrm{cm}^{2}}$, is

$$
\mathcal{N}=\frac{I \mathcal{V}}{\hbar \omega c}=\frac{\left(10^{10} \mathrm{~W} / \mathrm{cm}^{2}\right)\left(1 \mathrm{~cm}^{3}\right)}{\left(1.212 \times 10^{-19} \mathrm{~J}\right)\left(2.998 \times 10^{10 \frac{\mathrm{cm}}{\mathrm{s}}}\right)}=2.752 \times 10^{18} \text { photons }
$$

This gives a rate coefficent for stimulated attachment of 


$$
\alpha_{S A}(T=10 \mathrm{~K})=\left(2.752 \times 10^{18} \text { photons }\right)\left(1.070 \times 10^{-17} \frac{\mathrm{cm}^{3}}{\mathrm{~s}}\right) \approx 2.945 \times 10^{1} \frac{\mathrm{cm}^{3}}{\mathrm{~s}}
$$

The rate of stimulated attachment is

$$
\left.\frac{d \mathcal{N}_{H^{-}}}{d t}\right|_{t=0}=\alpha_{S A} n_{e} \mathcal{N}_{H}
$$

Therefore, we find

$$
\left.\frac{d \mathcal{N}_{H^{-}}}{d t}\right|_{t=0}=\left(2.945 \times 10^{1} \frac{\mathrm{cm}^{3}}{\mathrm{~s}}\right)\left(10^{10} \frac{\mathrm{e}^{-}}{\mathrm{cm}^{3}}\right)\left(10^{4} \mathrm{H}\right)=3 \times 10^{15} \frac{\mathrm{H}^{-}}{\mathrm{s}}
$$

This is a high rate of production. That rate is for continuous operation but a typical Ti-Sapphire pulse repetition rate is on the order of $70 \mathrm{MHz}$. That is a pulse repetition period of $15 \mathrm{~ns}$. This means the laser is only interacting with the hydrogen for about $5 \mu s$ out of each second of operation. This reduces the rate of production by a factor of $5 \times 10^{-6}$, giving a rate of about $1.5 \times 10^{10} \mathrm{~s}^{-1}$. This is still a decent rate of production. In order to maintain this rate, we would need to actively remove the product. If we cannot remove the product, then the system will eventually reach equilibrium. At equilibrium, we found

$$
n_{H^{-}}=\frac{\beta_{S A}}{\alpha_{P D}} n_{e} n_{H}=\frac{\left(1.070 \times 10^{-18} \frac{\mathrm{cm}^{6}}{\mathrm{~s} \cdot K} T\right)}{\left(1.230 \times 10^{-6} \frac{\mathrm{cm}}{\mathrm{s}}\right)} n_{e} n_{H}
$$

or

$$
n_{H^{-}}=n_{e} n_{H}\left(8.699 \times 10^{-13} \mathrm{~cm}^{3} K^{-1} T\right) .
$$

Thus, at $T=10 \mathrm{~K}$, we find at equilibrium 


$$
n_{H^{-}}=\left(10^{10} \frac{\mathrm{e}^{-}}{\mathrm{cm}^{3}}\right)\left(10^{4} \frac{\mathrm{H}}{\mathrm{cm}^{3}}\right)\left(8.699 \times 10^{-12} \mathrm{~cm}^{3}\right) \approx 1000 \frac{\mathrm{H}^{-}}{\mathrm{cm}^{3}}
$$

This amount of antihydrogen ions is observable. Next, we will look at a method that goes beyond perturbation theory. This will allow us to analyze laser intensities up to the point where the dipole approximation breaks down and should help increase rates. 


\section{Chapter 6}

\section{Beyond Perturbation}

\subsection{S-matrix and T-matrix}

In order to go beyond perturbation theory, we need to devise a general system for calculating quantum transition rates in which the mathematical environment is not built on a foundation of approximations. Luckily, Wheeler has devised just such a mathematical environment [52]. While Wheeler was interested in nuclear scattering (free-free transitions), the Scattering matrix (S-matrix) is a general system that can be applied to any system of intial and final states, such as bound-bound transition or free-bound transitions. Howard R. Reiss recognized the importance of this method of calculation for non-scattering (bound-free and free-bound) systems $[53,54,55,56$, 32, 26]. A less elegant, yet earlier, method was used by Keldysh [57].

The non-interacting solutions are given by the non-interacting Schrödinger equation,

$$
i \hbar \frac{\partial}{\partial t} \Psi^{(0)}=H_{0} \Psi^{(0)}
$$

and the interacting solutions are given by the interacting Schrödinger equation,

$$
i \hbar \frac{\partial}{\partial t} \Psi=\left(H_{0}+H_{i n t}\right) \Psi
$$


It is understood that the vector potential $\mathbf{A}$ satisfies the asymptotic condition

$$
\lim _{t \rightarrow \pm \infty} \mathbf{A}(t)=0 \Longrightarrow \lim _{t \rightarrow \pm \infty} H_{\text {int }}(t)=0
$$

That is, at some time in the long past the interaction was turned off, and at some time in the far future it is also off. Here "long" and "far" could be a femtosecond pulse! The atomic system was prepared initially in a well-defined non-interacting initial state $\Psi_{i}^{(0)}(t)$, such that

$$
\lim _{t \rightarrow-\infty} \Psi_{i}(t)=\Psi_{i}^{(0)}(t)
$$

The system also has a non-interacting final state $\Psi_{f}^{(0)}(\mathrm{t})$, such that

$$
\lim _{t \rightarrow \infty} \Psi_{f}(t)=\Psi_{f}^{(0)}(t)
$$

Then the Scattering matrix (S-matrix) can be defined as

$$
S_{f i}=\lim _{t \rightarrow \infty} \int \Psi_{f}^{(0) *}(t) \Psi_{i}(t) d^{3} x
$$

which is the amplitude of the non-interacting final state overlapped with the interacting initial state.

It is useful to define a Transition matrix (T-matrix)

$$
T_{f i}=S_{f i}-\delta_{f i}
$$

which simply subtracts off the probability of no transition from the S-matrix. Using, 
$\delta_{f i}=\int \Psi_{f}^{(0) *}(t) \Psi_{i}^{(0)}(t) d^{3} x$ and $\lim _{t \rightarrow-\infty} \Psi_{i}(t)=\Psi_{i}^{(0)}(t)$, the T-matrix can be written

$$
\begin{gathered}
T_{f i}=\lim _{t \rightarrow \infty} \int \Psi_{f}^{(0) *}(t) \Psi_{i}(t) d^{3} x-\int \Psi_{f}^{(0) *}(t) \Psi_{i}^{(0)}(t) d^{3} x \\
=\lim _{t \rightarrow \infty} \int \Psi_{f}^{(0) *}(t) \Psi_{i}(t) d^{3} x-\lim _{t \rightarrow-\infty} \int \Psi_{f}^{(0) *}(t) \Psi_{i}(t) d^{3} x \\
=\frac{\partial}{\partial t} \iint \Psi_{f}^{(0) *}(t) \Psi_{i}(t) d^{3} x d t
\end{gathered}
$$

where in the last step we applied the fundamental theorem of calculus. Using the Schrödinger equation to replace time derivative with Hamiltonians,

$$
T_{f i}=-\frac{i}{\hbar} \iint\left[H_{0} \Psi_{f}^{(0) *}(t)\right] \Psi_{i}(t) d^{3} x d t-\frac{i}{\hbar} \iint \Psi_{f}^{(0) *}(t)\left(H_{0}+H_{i n t}\right) \Psi_{i}(t) d^{3} x d t
$$

Using integration by parts (or Hemiticity of $H_{0}$ ) on the first term,

$$
T_{f i}=\frac{i}{\hbar} \iint \Psi_{f}^{(0) *}(t) H_{0} \Psi_{i}(t) d^{3} x d t-\frac{i}{\hbar} \iint \Psi_{f}^{(0) *}(t)\left(H_{0}+H_{i n t}\right) \Psi_{i}(t) d^{3} x d t
$$

and noting the first two terms with integrals of $H_{0}$ cancel,

$$
T_{f i}=-\frac{i}{\hbar} \iint \Psi_{f}^{(0) *}(t) H_{i n t} \Psi_{i}(t) d^{3} x d t
$$

This gives us a S-matrix

$$
S_{f i}=\delta_{f i}-\frac{i}{\hbar} \iint \Psi_{f}^{(0) *}(t) H_{\text {int }}(t) \Psi_{i}(t) d^{3} x d t
$$

We note that no approximations were used in this derivation. These are the general 
quantum S-matrix and T-matrix. They are exact. This is the basis on which the Strong Field Approximation is built.

The equations of motion are invariant under time reversal; therefore, we can form the fully equivalent S-matrices and T-matrices,

$$
\begin{gathered}
S_{f i}=\lim _{t \rightarrow-\infty} \int \Psi_{f}^{*}(t) \Psi_{i}^{(0)}(t) d^{3} x . \\
T_{f i}=-\frac{i}{\hbar} \iint \Psi_{f}^{*}(t) H_{\text {int }} \Psi_{i}^{(0)}(t) d^{3} x d t .
\end{gathered}
$$

We will now continue on to the Strong Field Approximation.

\subsection{Strong Field Approximation (SFA)}

If the applied laser field is sufficiently large, then a perturbation expansion will simply not converge. It is thus necessary to have other methods to extend our knowledge of the behavior of atoms in intense laser fields. The Strong Field Approximation (SFA) is a nonperturbative, analytical approximation that leads naturally to formulations of transition amplitudes. It is formulated in the time-reversed transition amplitude where the interacting state is the final state, rather than the intitial state. For the Hydrogen atom, one can put a limit for when the SFA begins to break down. This condition is when the Coulomb interaction (or, in our case, finite range interaction) starts to become as prominent as the external field. Thus,

$$
E_{B} \gg U_{p},
$$

where $E_{B}$ is the binding energy of the electron. This leads to

$$
I_{S F A}=\frac{2 \epsilon_{0} m c}{e^{2}} E_{B} \omega^{2}=\left(\frac{E_{B}}{\hbar \omega}\right) \frac{1}{\alpha} \frac{1}{2 \pi} m \omega^{3} .
$$


Thus, with a Ti-Sapphire laser $(\lambda=800 \mathrm{~nm})$ and $E_{B} \approx 1 \mathrm{eV}$, the assumptions of the SFA should begin to weaken at intensities

$$
I \ll I_{S F A} \approx 1.6 \times 10^{13} \mathrm{~W} / \mathrm{cm}^{2} .
$$

However, this quantity has a history of misinterpretations. Is it related to tunneling in anyway? In fact, this has been shown not to be the case. Is it the limit where multiphoton interactions begin to overlap? Perhaps. At any rate, one can see that this approximation is good for our system with a loosely bound electron where we have a neutral target and the proton is almost entirely screened by a bound electron. This quantity is sometimes presented as a unitless parameter in literature called the field-dominated intensity parameter,

$$
z_{1}=\frac{2 U_{p}}{E_{B}} .
$$

The SFA can be thought of as a zeroth order perturbation of the Coulomb interaction of the free electron with the nucleus. Zeroth order meaning that we ignore the interaction with the nucleus. The exact wavefunction would take into account both interaction of the electron with the laser field and the nucleus. The exact transition amplitude is

$$
T_{f i}=-\frac{i}{\hbar} \iint \Psi_{f}^{*}(t) H_{i n t} \Psi_{i}^{(0)}(t) d^{3} x d t .
$$

In the case of photodetachment, the state $\Psi_{f}(t)$ is the fully detached state. The Strong Field Approximation statement can be summarized by saying, "in the continuum 'free' electron state assume that the laser field is sufficiently strong so as to dominate over the effects of the atomic potential which are 'far away."' In other words, we will assume the Coulomb potential has no effect on the detached electron. Therefore, 
(ignoring the other possible electrons for the moment), we approximate $\Psi_{f}(t)$ by the Volkov solution for a "free" particle in a field. Namely

$$
\Psi_{f}(t) \approx \chi(\mathbf{r}, t)=\frac{1}{\sqrt{\mathcal{V}}} \exp (-i \mathbf{k} \cdot \mathbf{r}) \exp \left[\frac{i}{\hbar}\left(E_{k} t+\int V_{i n t}\left(t^{\prime}\right) d t^{\prime}\right)\right]
$$

so that the T-matrix is

$$
T_{f i}=-\frac{i}{\hbar} \iint \chi^{*}(\mathbf{r}, t) H_{\text {int }} \Psi_{i}^{(0)}(t) d^{3} x d t .
$$

This is the (zeroth order) Strong Field Approximation. It is an interesting fact that because we choose in our calculations to use the finite range approximation, all higher

order corrections factors are in fact zero. That is, within our finite range approximation, the two electrons wavefunction are completely separable. Therefore, the "free" electron only feels the laser field and does not feel the atom. The bound electron only feels the nucleus and laser field. This means, as we will soon see, that our calculation will "work" for intensities down to zero. We will not be constrained by the field-dominated intensity parameter. This would not be the case had we used the exact Coulomb potential.

\subsection{Applicaton of the Strong Field Approximation}

Starting with photodetachment of two particle systems, the final state will be a Volkov particle and bound particle $[59,58,60]$,

$$
\Psi_{f}\left(\mathbf{r}_{1}, \mathbf{r}_{2}, t\right)=\frac{1}{\sqrt{2}}\left[\chi\left(\mathbf{r}_{1}, t\right) \Psi_{1 s}\left(\mathbf{r}_{2}, t\right)+\chi\left(\mathbf{r}_{2}, t\right) \Psi_{1 s}\left(\mathbf{r}_{1}, t\right)\right] .
$$


The initial state will be the ion,

$$
\Psi_{i}^{(0)}\left(\mathbf{r}_{1}, \mathbf{r}_{2}, t\right)= \begin{cases}\psi_{\text {eff }}\left(\mathbf{r}_{1}\right) \psi_{1 s}\left(\mathbf{r}_{2}\right) \exp \left(-\frac{i}{\hbar} E_{\text {ion }} t\right) & r_{1}>r_{2} \\ \psi_{\text {eff }}\left(\mathbf{r}_{2}\right) \psi_{1 s}\left(\mathbf{r}_{1}\right) \exp \left(-\frac{i}{\hbar} E_{\text {ion }} t\right) & r_{2}>r_{1}\end{cases}
$$

where

$$
E_{i o n}=E_{1 s}+E_{e f f}
$$

The T-matrix simplifies to

$$
T_{f i}=-\frac{i}{\hbar} \iint \chi^{*}(\mathbf{r}, t) H_{i n t} \psi_{e f f}(\mathbf{r}) \exp \left(\frac{i}{\hbar} E_{B} t\right) d^{3} x d t
$$

where

$$
E_{B}=E_{1 s}-E_{i o n}
$$

We point out that $E_{i o n}$ is a more negative number than $E_{1 s}$, making the binding energy $E_{B}$ a positive quantity by convention (i.e. $E_{B}=-E_{\text {eff }}$ ). The term $H_{\text {int }} \Psi_{\text {ion }}$ is not very easily worked out; therefore, we carry out an integration by parts over $t$. The T-matrix for this situation is

$$
\begin{gathered}
T_{f i}= \\
-\frac{i}{\hbar} \iint \frac{1}{\sqrt{\mathcal{V}}} \exp (-i \mathbf{k} \cdot \mathbf{r}) \exp \left[\frac{i}{\hbar}\left(E_{k} t+\int V_{i n t}\left(t^{\prime}\right) d t^{\prime}\right)\right] H_{i n t} \psi_{e f f}(\mathbf{r}) \exp \left(\frac{i}{\hbar} E_{B} t\right) d^{3} x d t
\end{gathered}
$$

We let

$$
\begin{gathered}
u=-\frac{1}{\sqrt{\mathcal{V}}} \exp \left(\frac{i}{\hbar} E_{k} t\right) \exp \left(\frac{i}{\hbar} E_{B} t\right) \exp (-i \mathbf{k} \cdot \mathbf{r}) \\
d u=-\frac{1}{\sqrt{\mathcal{V}}} \frac{i}{\hbar}\left(E_{k}+E_{B}\right) \exp \left(\frac{i}{\hbar} E_{k} t\right) \exp \left(\frac{i}{\hbar} E_{B} t\right) \exp (-i \mathbf{k} \cdot \mathbf{r}) d t \\
d v=\frac{i}{\hbar} \exp \left[\frac{i}{\hbar} \int_{-\infty}^{t} V_{i n t}\left(t^{\prime}\right) d t^{\prime}\right] H_{\text {int }} \psi_{\text {eff }}(\mathbf{r}) d t
\end{gathered}
$$




$$
v=\exp \left[\frac{i}{\hbar} \int_{-\infty}^{t} V_{i n t}\left(t^{\prime}\right) d t^{\prime}\right] \psi_{e f f}(\mathbf{r})
$$

.We note that integration parts over $t$ is useful when we choose the Volkov wavefunction for the final state. It does not produce any advantage with a planewave wavefunction. Integration by parts states that $\int u d v=-\int v d u$ (because $\left.u v\right|_{\text {limits }}=0$ ) so that

$$
\begin{aligned}
& -\int \frac{1}{\sqrt{\mathcal{V}}} \exp \left(\frac{i}{\hbar} E_{k} t\right) \exp \left(\frac{i}{\hbar} E_{B} t\right) \exp (-i \mathbf{k} \cdot \mathbf{r}) \frac{i}{\hbar} \exp \left[\frac{i}{\hbar}\left(E_{k} t+\int V_{\text {int }}\left(t^{\prime}\right) d t^{\prime}\right)\right] H_{\text {int }} \psi_{\text {eff }}(\mathbf{r}) d t \\
& =\int \exp \left[\frac{i}{\hbar} \int V_{\text {int }}\left(t^{\prime}\right) d t^{\prime}\right] \psi_{\text {eff }}(\mathbf{r}) \frac{1}{\sqrt{\mathcal{V}}} \frac{i}{\hbar}\left(E_{k}+E_{B}\right) \exp \left(\frac{i}{\hbar} E_{k} t\right) \exp \left(\frac{i}{\hbar} E_{B} t\right) \exp (-i \mathbf{k} \cdot \mathbf{r}) d t .
\end{aligned}
$$

This transforms the T-matrix,

$$
\begin{gathered}
T_{f i}= \\
\frac{1}{\sqrt{\mathcal{V}}} \frac{i}{\hbar}\left(E_{k}+E_{B}\right) \int \exp (-i \mathbf{k} \cdot \mathbf{r}) \psi_{e f f}(\mathbf{r}) d^{3} x \int \exp \left[\frac{i}{\hbar} \int V_{i n t}\left(t^{\prime}\right) d t^{\prime}\right] \exp \left(\frac{i}{\hbar} E_{k} t\right) \exp \left(\frac{i}{\hbar} E_{B} t\right) d t
\end{gathered}
$$

to

$$
T_{f i}=\frac{i}{\hbar}\left(E_{k}+E_{B}\right) \widetilde{\psi}_{e f f}(\mathbf{k}) \int \exp \left[\frac{i}{\hbar}\left(\left(E_{k}+E_{B}\right) t\right)\right] \exp \left[\frac{i}{\hbar} \int V_{i n t}\left(t^{\prime}\right) d t^{\prime}\right] d t
$$

where $\widetilde{\psi}_{\text {eff }}(\mathbf{k})$ is the Fourier transform of $\psi_{\text {eff }}(\mathbf{r})$,

$$
\widetilde{\psi}_{e f f}(\mathbf{k})=\frac{1}{\sqrt{\mathcal{V}}} \int \exp (-i \mathbf{k} \cdot \mathbf{r}) \psi_{e f f}(\mathbf{r}) d^{3} x
$$

Focusing our attention on the term with the interaction potential, we have

$$
\frac{1}{\hbar} \int V_{i n t}\left(t^{\prime}\right) d t^{\prime}=\int\left(\frac{e}{m} \mathbf{k} \cdot \mathbf{A}\left(t^{\prime}\right)+\frac{e^{2}}{2 m \hbar} \mathbf{A}^{2}\left(t^{\prime}\right)\right) d t^{\prime}
$$




\section{FIGURE-8 MOTION OF A FREE ELECTRON IN A}

\section{LINEARLY POLARIZED PLANE-WAVE FIELD}

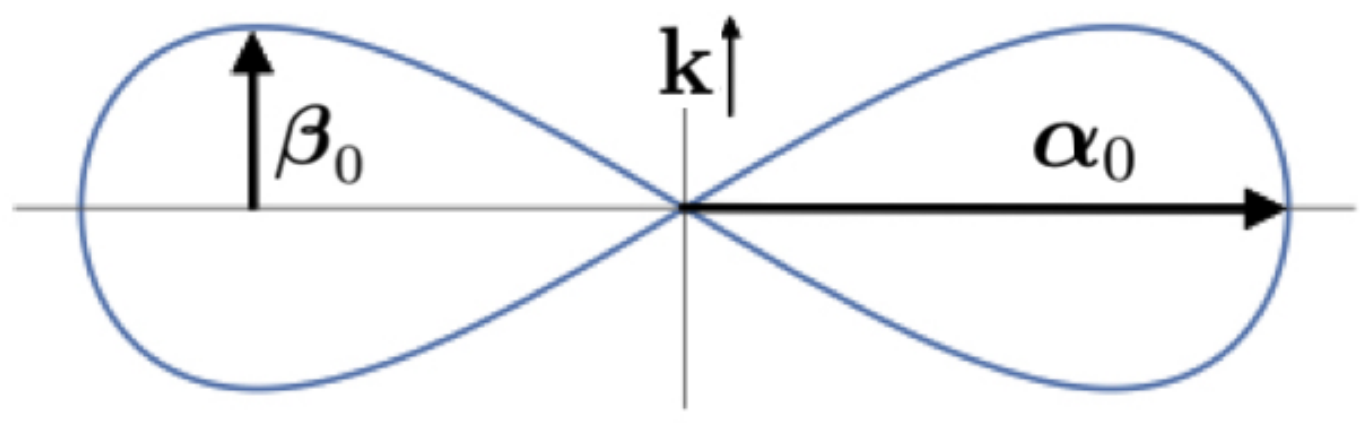

Figure 6.1: The quiver amplitude $\boldsymbol{\alpha}_{0}$, the shiver amplitude $\boldsymbol{\beta}_{0}$, and the direction of propagation $\mathbf{k}$.

$$
\begin{gathered}
=\int\left(\frac{e}{m} A_{0} \cos \left(\omega t^{\prime}\right) \mathbf{k} \cdot \hat{\boldsymbol{\epsilon}}+\frac{e^{2}}{2 m \hbar} A_{0}^{2} \cos ^{2}\left(\omega t^{\prime}\right)\right) d t^{\prime} \\
=\mathbf{k} \cdot\left(\frac{e A_{0}}{m \omega} \hat{\boldsymbol{\epsilon}}\right) \sin (\omega t)+k \frac{e^{2} A_{0}^{2}}{8 m \hbar \omega k} \sin (2 \omega t)+k \frac{e^{2} A_{0}^{2}}{4 m \hbar \omega k} \omega t \\
=\mathbf{k} \cdot \boldsymbol{\alpha}_{0} \sin (\omega t)+k \beta_{0} \sin (2 \omega t)+2 k \beta_{0} \omega t .
\end{gathered}
$$

It is useful to define the quiver motion of the electron in the laser field (not to be confused with $\alpha$, the fine structure constant) as

$$
\boldsymbol{\alpha}(t)=\frac{e}{m} \int \mathbf{A}\left(t^{\prime}\right) d t^{\prime}=\boldsymbol{\alpha}_{0} \sin (\omega t)
$$

where

$$
\boldsymbol{\alpha}_{0}=\frac{e A_{0}}{m \omega} \hat{\boldsymbol{\epsilon}}=\frac{e E_{0}}{m \omega^{2}} \hat{\boldsymbol{\epsilon}}
$$


and

$$
\alpha_{0}=\frac{e A_{0}}{m \omega}=\frac{e E_{0}}{m \omega^{2}}=\sqrt{\frac{4 \hbar z}{m \omega}}=\sqrt{\frac{4 U_{p}}{m \omega^{2}}} .
$$

The quiver (amplitude) is simply the amplitude of the motion of an electron in a laser field along the direction of the electric field. That is to say, the quivering electron moves in the $\hat{\boldsymbol{\epsilon}}$ direction, extending back and forth between $-\alpha_{0}$ and $\alpha_{0}$. The quantity

$$
\beta_{0}=\frac{e^{2} A_{0}^{2}}{8 m \hbar \omega k}
$$

is called the shiver. The shiver is the amplitude of the motion of an electron in a laser field along the direction of propagation and is twice the frequancy as the quiver motion. This implies that a "free" electron in a plane-wave field will move with a figure-8 pattern. It is convenient to also define the unitless quantity

$$
z=2 k \beta_{0}=\frac{e^{2} A_{0}^{2}}{4 m \hbar \omega}=\frac{U_{p}}{\hbar \omega}=\frac{I}{I_{0}} .
$$

This quantity is called the non-perturbative intensity parameter. Its importance has already been discussed. In order for a perturbative series to converge $z \ll 1$.

At this point it is neccessary to define $J_{n}(x, y)$ the generalized Bessel function. Integral representation:

$$
J_{n}(x, y)=\frac{1}{2 \pi} \int_{-\pi}^{\pi} \exp [i(x \sin (\theta)+y \sin (2 \theta)-n \theta)] d \theta
$$

Series representation:

$$
J_{n}(x, y)=\sum_{k=-\infty}^{\infty} J_{n-2 k}(x) J_{k}(y)
$$


Generating function:

$$
\exp [i(x \sin (\theta)+y \sin (2 \theta))]=\sum_{n=-\infty}^{\infty} e^{i n \theta} J_{n}(x, y)
$$

where $J_{n}(x)$ is the ordinary Bessel function. We also point out the useful properties

$$
\begin{gathered}
J_{-n}(x, y)=(-1)^{n} J_{n}(x,-y)=J_{n}(-x,-y), \\
J_{n}(x, y) \approx J_{n}(x)+\frac{1}{2} y\left[J_{n-2}(x)-J_{n+2}(x)\right] \quad|y| \ll 1,
\end{gathered}
$$

and

$$
\begin{gathered}
J_{1}(x, y) \approx \frac{x}{2} \quad x,|y| \ll 1 . \\
J_{2}(x, y) \approx \frac{y}{2}+\frac{1}{2}\left(\frac{x}{2}\right)^{2} \quad x,|y| \ll 1 .
\end{gathered}
$$

For large argument $y \gg 1$, the asymptotic approximation is (see H.J. Korsch [61])

$$
J_{n}(x, y) \rightarrow\left\{\begin{array}{cc}
\sqrt{\frac{2}{\pi y}} \cos \left(\frac{x}{\sqrt{2}}\right) \cos \left(y-(n+1) \frac{\pi}{4}\right) & n \text { even } \\
-\sqrt{\frac{2}{\pi y}} \sin \left(\frac{x}{\sqrt{2}}\right) \sin \left(y-(n+1) \frac{\pi}{4}\right) & n \text { odd }
\end{array}\right.
$$

except in the vicinity of $y=\frac{x}{2}$ where the asymptotic approximation diverges. Or for negative the second argument, $y \ll-1$,

$$
J_{n}(x, y) \rightarrow\left\{\begin{array}{cc}
-i \sqrt{\frac{2}{\pi y}} \cos \left(\frac{x}{\sqrt{2}}\right) \cos \left(y-(n-1) \frac{\pi}{4}\right) & n \text { even } \\
i \sqrt{\frac{2}{\pi y}} \sin \left(\frac{x}{\sqrt{2}}\right) \sin \left(y-(n-1) \frac{\pi}{4}\right) & n \text { odd }
\end{array}\right.
$$

In the notation of the generalized Bessel, the T-matrix is

$$
T_{f i}=
$$




$$
\frac{i}{\hbar}\left(E_{k}+E_{B}\right) \widetilde{\psi}_{e f f}(\mathbf{k}) \int \exp \left[\frac{i}{\hbar}\left(\left(E_{k}+E_{B}+U_{p}\right) t\right)\right] \sum_{n=-\infty}^{\infty} e^{-i n \omega t}(-1)^{n} J_{n}\left(\mathbf{k} \cdot \boldsymbol{\alpha}_{0},-\frac{z}{2}\right) d t
$$

or

$$
T_{f i}=\sum_{n=-\infty}^{\infty} 2 \pi i\left(E_{k}+E_{B}\right) \widetilde{\psi}_{e f f}(\mathbf{k})(-1)^{n} J_{n}\left(\mathbf{k} \cdot \boldsymbol{\alpha}_{0},-\frac{z}{2}\right) \delta\left(E_{k}+E_{B}+U_{p}-n \hbar \omega\right)
$$

The average rate of transition can be expressed as

$$
\Gamma_{f i}=\frac{\left|T_{f i}\right|^{2}}{t}
$$

Focusing our attention on the term $\left|T_{f i}\right|^{2}$, we have

$$
\begin{gathered}
\left|T_{f i}\right|^{2}=\sum_{n^{\prime}=-\infty}^{\infty} \sum_{n=-\infty}^{\infty}(2 \pi)^{2}\left(E_{k}+E_{B}\right)^{2}\left|\widetilde{\psi}_{e f f}(\mathbf{k})\right|^{2} J_{n^{\prime}}\left(\mathbf{k} \cdot \boldsymbol{\alpha}_{0},-z\right) J_{n}\left(\mathbf{k} \cdot \boldsymbol{\alpha}_{0},-\frac{z}{2}\right) \\
\times \delta\left(E_{k}+E_{B}+U_{p}-n^{\prime} \hbar \omega\right) \delta\left(E_{k}+E_{B}+U_{p}-n \hbar \omega\right) .
\end{gathered}
$$

Using the fact,

$$
f(x) \delta\left(x-x_{0}\right)=f\left(x_{0}\right) \delta\left(x-x_{0}\right)
$$

we find,

$$
\begin{gathered}
\left|T_{f i}\right|^{2}=\sum_{n^{\prime}=-\infty}^{\infty} \sum_{n=-\infty}^{\infty}(2 \pi)^{2}\left(E_{k}+E_{B}\right)^{2}\left|\widetilde{\psi}_{e f f}(\mathbf{k})\right|^{2} \\
\times J_{n^{\prime}}\left(\mathbf{k} \cdot \boldsymbol{\alpha}_{0},-\frac{z}{2}\right) J_{n}\left(\mathbf{k} \cdot \boldsymbol{\alpha}_{0},-\frac{z}{2}\right) \delta\left(\left(n-n^{\prime}\right) \hbar \omega\right) \delta\left(E_{k}+E_{B}+U_{p}-n \hbar \omega\right) .
\end{gathered}
$$

We note that the only non-zero terms in this series are when $n-n^{\prime}=0$. Therefore,

$$
\left|T_{f i}\right|^{2}=\sum_{n=-\infty}^{\infty}(2 \pi)^{2}\left(E_{k}+E_{B}\right)^{2}\left|\widetilde{\psi}_{e f f}(\mathbf{k})\right|^{2} J_{n}^{2}\left(\mathbf{k} \cdot \boldsymbol{\alpha}_{0},-\frac{z}{2}\right) \delta(0) \delta\left(E_{k}+E_{B}+U_{p}-n \hbar \omega\right) .
$$


Assume that the interaction time (although large) is finite then

$$
\left|T_{f i}\right|^{2}=\sum_{n=-\infty}^{\infty}(2 \pi)^{2}\left(E_{k}+E_{B}\right)^{2}\left|\widetilde{\psi}_{e f f}(\mathbf{k})\right|^{2} J_{n}^{2}\left(\mathbf{k} \cdot \boldsymbol{\alpha}_{0},-\frac{z}{2}\right) \frac{t}{2 \pi \hbar} \delta\left(E_{k}+E_{B}+U_{p}-n \hbar \omega\right) .
$$

This gives us the average rate of the transition as

$$
\Gamma_{f i}=\sum_{n=-\infty}^{\infty} \frac{2 \pi}{\hbar}\left(E_{k}+E_{B}\right)^{2}\left|\widetilde{\psi}_{e f f}(\mathbf{k})\right|^{2} J_{n}^{2}\left(\mathbf{k} \cdot \boldsymbol{\alpha}_{0},-\frac{z}{2}\right) \delta\left(E_{k}+E_{B}+U_{p}-n \hbar \omega\right) .
$$

This expression is something like a Golden Rule for Strong Fields. Now we turn our attention to the delta function $\delta\left(E_{k}+E_{B}+U_{p}-n \hbar \omega\right)$. Using

$$
\delta\left(E_{k}+E_{B}+U_{p}-n \hbar \omega\right)=\frac{m}{p} \delta\left(p-\sqrt{2 m\left(n \hbar \omega-\left(E_{B}+U_{p}\right)\right)}\right)
$$

gives us the expression

$$
\begin{gathered}
\Gamma_{P D}=\sum_{f} \Gamma_{f i}=\int \Gamma_{f i} d^{3} n_{f}= \\
\int\left(\sum_{n=-\infty}^{\infty} \frac{2 \pi}{\hbar}\left(E_{k}+E_{B}\right)^{2}\left|\widetilde{\psi}_{e f f}(\mathbf{k})\right|^{2} J_{n}^{2}\left(\mathbf{k} \cdot \boldsymbol{\alpha}_{0},-\frac{z}{2}\right) \frac{m}{p} \delta\left(p-\sqrt{2 m\left(n \hbar \omega-\left(E_{B}+U_{p}\right)\right)}\right)\right) \\
\times \frac{\mathcal{V}}{(2 \pi)^{3} \hbar^{3}} p^{2} d p d \Omega .
\end{gathered}
$$

Upon integrating over momentum of the ejected electron, we find

$$
\Gamma_{P D}=\sum_{n=n_{0}}^{\infty} \frac{\mathcal{V} m}{(2 \pi)^{2} \hbar^{4}} p\left(E_{k}+E_{B}\right)^{2}\left|\widetilde{\psi}_{e f f}(\mathbf{k})\right|^{2} \int J_{n}^{2}\left(\mathbf{k} \cdot \boldsymbol{\alpha}_{0},-\frac{z}{2}\right) d \Omega
$$


or the differential cross-section

$$
\frac{d \Gamma_{P D}}{d \Omega}=\sum_{n=n_{0}}^{\infty} \frac{\mathcal{V} m}{(2 \pi)^{2} \hbar^{4}} p\left(E_{k}+E_{B}\right)^{2}\left|\widetilde{\psi}_{e f f}(\mathbf{k})\right|^{2} J_{n}^{2}\left(\mathbf{k} \cdot \boldsymbol{\alpha}_{0},-\frac{z}{2}\right),
$$

where now $p=\sqrt{2 m\left(n \hbar \omega-\left(E_{B}+U_{p}\right)\right)}$ and we have dropped subscripts.

In order for the electron to be photodetached, we need to get enough energy from the field in the form of photons $(n \hbar \omega)$ to get out of the bound state and begin its quiver and shiver motion. The minimum number of photons needed in order to begin detachment is set by

$$
n_{0}=\left\lceil\frac{E_{B}+U_{p}}{\hbar \omega}\right\rceil=\left\lceil\frac{E_{B}}{\hbar \omega}+z\right\rceil,
$$

where the upper bracket notation $\lceil x\rceil$ means to round $x$ up to the next integer. For most frequencies (wavelengths) and intensities, the mininum number of photons needed will simply be $n_{0}=1$. In our case,

$$
n_{0}=\left\lceil\frac{.75 \mathrm{eV}}{1.5 \mathrm{eV}}+z\right\rceil=\lceil .5+z\rceil,
$$

Thus $n_{0}=1$ up to intensities of $z \approx .5$. After that, for intensities between $.5 \lesssim$ $z \lesssim 1.5, n_{0}=2$. and for intensities $1.5 \lesssim z \lesssim 2.5, n_{0}=3$. This is a result of the one photon transition not being energetically possible due to the high ponderomotive energy.

For large intensities, it is sometimes convenient to define $s=n-n_{0}$, so that

$$
\frac{d \Gamma_{P D}}{d \Omega}=\sum_{s=0}^{\infty} \frac{\mathcal{V} m}{(2 \pi)^{2} \hbar^{4}} p\left(E_{k}+E_{B}\right)^{2}\left|\widetilde{\psi}_{e f f}(\mathbf{k})\right|^{2} J_{n_{0}+s}^{2}\left(\mathbf{k} \cdot \boldsymbol{\alpha}_{0},-\frac{z}{2}\right),
$$

with the summation starting from zero. 


\subsection{The Weak Field Limit}

Now, we will check the weak field limit. For weak fields (i.e. $\mathbf{k} \cdot \boldsymbol{\alpha}_{0} \ll 1$ and $z \ll 1$ ),

$$
J_{1}^{2}\left(\mathbf{k} \cdot \boldsymbol{\alpha}_{0},-z\right) \approx\left(\frac{\mathbf{k} \cdot \boldsymbol{\alpha}_{0}}{2}\right)^{2}
$$

which is proportional to, $I$, the intensity. In the weak field limit, we find the differential rate to be

$$
\frac{d \Gamma_{P D}}{d \Omega} \approx \frac{\mathcal{V} m}{(2 \pi)^{2} \hbar^{4}} p\left(E_{k}+E_{B}\right)^{2}\left|\widetilde{\psi}_{e f f}(\mathbf{k})\right|^{2}\left(\frac{\mathbf{k} \cdot \boldsymbol{\alpha}_{0}}{2}\right)^{2}
$$

Thus, for sinisoidal fields the differential cross-section is

$$
\frac{d \sigma_{P D}}{d \Omega}=\sum_{n=n_{0}}^{\infty} \frac{\hbar \omega}{\frac{1}{2} \epsilon_{0} c \omega^{2} A_{0}^{2}} \frac{\mathcal{V} m}{(2 \pi)^{2} \hbar^{4}} p\left(E_{k}+E_{B}\right)^{2}\left|\widetilde{\psi}_{e f f}(\mathbf{k})\right|^{2}\left(\frac{\mathbf{k} \cdot \boldsymbol{\alpha}_{0}}{2}\right)^{2} .
$$

This simplifies to

$$
\sigma_{P D} \approx \alpha \frac{32 \pi}{3} \mathcal{C}^{2} \gamma \frac{k^{3}}{\left(k^{2}+\gamma^{2}\right)^{3}}
$$

or

$$
\sigma_{P D} \approx \alpha \frac{32 \pi}{3} \mathcal{C}^{2} a_{0}^{2}\left(\gamma a_{0}\right) \frac{\left(k a_{0}\right)^{3}}{\left(\left(k a_{0}\right)^{2}+\left(\gamma a_{0}\right)^{2}\right)^{3}},
$$

reproducing the result of Chapter 5 using first order perturbation. We note that the next term in the series has

$$
\begin{aligned}
& J_{2}^{2}\left(\mathbf{k} \cdot \boldsymbol{\alpha}_{0},-\frac{z}{2}\right) \approx\left(\frac{1}{2}\left(-\frac{z}{2}\right)+\frac{1}{2}\left(\frac{\mathbf{k} \cdot \boldsymbol{\alpha}_{0}}{2}\right)^{2}\right)^{2} \\
= & \frac{1}{4}\left(-\frac{z}{2}\right)^{2}+\left(-\frac{z}{2}\right) \frac{1}{2}\left(\frac{\mathbf{k} \cdot \boldsymbol{\alpha}_{0}}{2}\right)^{2}+\frac{1}{4}\left(\frac{\mathbf{k} \cdot \boldsymbol{\alpha}_{0}}{2}\right)^{4}
\end{aligned}
$$

which is proportional to $I^{2}$, consistent with second order perturbation theory. 


\subsection{The Function $\Xi_{n}\left(k \alpha_{0},-\frac{z}{2}\right)$}

In order to proceed with the analysis of rates in strong fields, we will need to tackle the integral over the solid angle. In strong fields the rate is

$$
\Gamma_{P D}=\sum_{n=n_{0}}^{\infty} \frac{\mathcal{V} m}{(2 \pi)^{2} \hbar^{4}} p\left(E_{k}+E_{B}\right)^{2}\left|\widetilde{\psi}_{e f f}(\mathbf{k})\right|^{2} \int J_{n}^{2}\left(\mathbf{k} \cdot \boldsymbol{\alpha}_{0},-\frac{z}{2}\right) d \Omega
$$

while cross section is

$$
\sigma_{P D}=\sum_{n=n_{0}}^{\infty} \frac{\hbar \omega}{\frac{1}{2} \epsilon_{0} c \omega^{2} A_{0}^{2}} \frac{\mathcal{V} m}{(2 \pi)^{2} \hbar^{4}} p\left(E_{k}+E_{B}\right)^{2}\left|\widetilde{\psi}_{e f f}(\mathbf{k})\right|^{2} \int J_{n}^{2}\left(\mathbf{k} \cdot \boldsymbol{\alpha}_{0},-\frac{z}{2}\right) d \Omega
$$

Both require the integral of the square of the generalized Bessel function over the solid angle. Let us define,

$$
\Xi_{n}\left(k \alpha_{0},-\frac{z}{2}\right) \equiv \int J_{n}^{2}\left(\mathbf{k} \cdot \boldsymbol{\alpha}_{0},-\frac{z}{2}\right) d \Omega,
$$

focusing our analysis on this function. This integral can be solved analytically by expanding the generalized Bessel function in terms of spherical harmonics

$$
J_{n}\left(\mathbf{k} \cdot \boldsymbol{\alpha}_{0},-\frac{z}{2}\right)=\sum_{l m} F_{l m}^{n}\left(k \alpha_{0},-\frac{z}{2}\right) Y_{l m}(\theta, \phi) .
$$

The analytic solution can be summarized by 


$$
\begin{aligned}
& \Xi_{n}\left(k \alpha_{0},-\frac{z}{2}\right)=\sum_{l=0}^{\infty}\left|F_{n l}\left(k \alpha_{0},-\frac{z}{2}\right)\right|^{2} \\
& F_{n l}\left(k \alpha_{0},-\frac{z}{2}\right)=\sum_{\eta=-\infty}^{\infty} \mathbb{J}_{n-2 \eta, l}\left(k \alpha_{0}\right) J_{\eta}\left(-\frac{z}{2}\right) \\
& \mathbb{J}_{n-2 \eta, l}\left(k \alpha_{0}\right)=(-1)^{l} \sqrt{\frac{2 l+1}{4 \pi}} \mathcal{J}_{n-2 \eta, l}\left(k \alpha_{0}\right) \\
& \mathcal{J}_{n^{\prime} l}(x)=\left\{\begin{array}{cc}
2 i^{l-n^{\prime}}{ }_{\pi^{3}}{ }^{2} \sum_{k=0}^{\infty} \frac{(-1)^{k}}{k ! \Gamma(k+l+3 / 2)}\left(\begin{array}{c}
2 k+l \\
\frac{2 k+l+\left|n^{\prime}\right|}{2}
\end{array}\right)\left(\frac{x}{4}\right)^{2 k+l} & \bmod \left(n^{\prime}+l, 2\right)=0 \\
0 & \bmod \left(n^{\prime}+l, 2\right) \neq 0
\end{array}\right.
\end{aligned}
$$

For details about the derivation of these functions see Appendix D. This gives us a strong field rate of

$$
\Gamma_{P D}=\sum_{n=n_{0}}^{\infty} \frac{\mathcal{V} m}{(2 \pi)^{2} \hbar^{4}} p\left(E_{k}+E_{B}\right)^{2}\left|\widetilde{\psi}_{e f f}(\mathbf{k})\right|^{2} \Xi_{n}\left(k \alpha_{0},-\frac{z}{2}\right)
$$

and cross section

$$
\sigma_{P D}=\sum_{n=n_{0}}^{\infty} \frac{\hbar \omega}{\frac{1}{2} \epsilon_{0} c \omega^{2} A_{0}^{2}} \frac{\mathcal{V} m}{(2 \pi)^{2} \hbar^{4}} p\left(E_{k}+E_{B}\right)^{2}\left|\widetilde{\psi}_{e f f}(\mathbf{k})\right|^{2} \Xi_{n}\left(k \alpha_{0},-\frac{z}{2}\right)
$$

\subsection{Strong Field Photodetachment (PD)}

Using the function $\Xi_{n}\left(k \alpha_{0},-\frac{z}{2}\right)$, the strong field rate is

$$
\Gamma_{P D}=\sum_{n=n_{0}}^{\infty} \frac{1}{2 \pi} \mathcal{C}^{2} \frac{p_{0}}{m} k \Xi_{n}\left(k \alpha_{0},-\frac{z}{2}\right)
$$




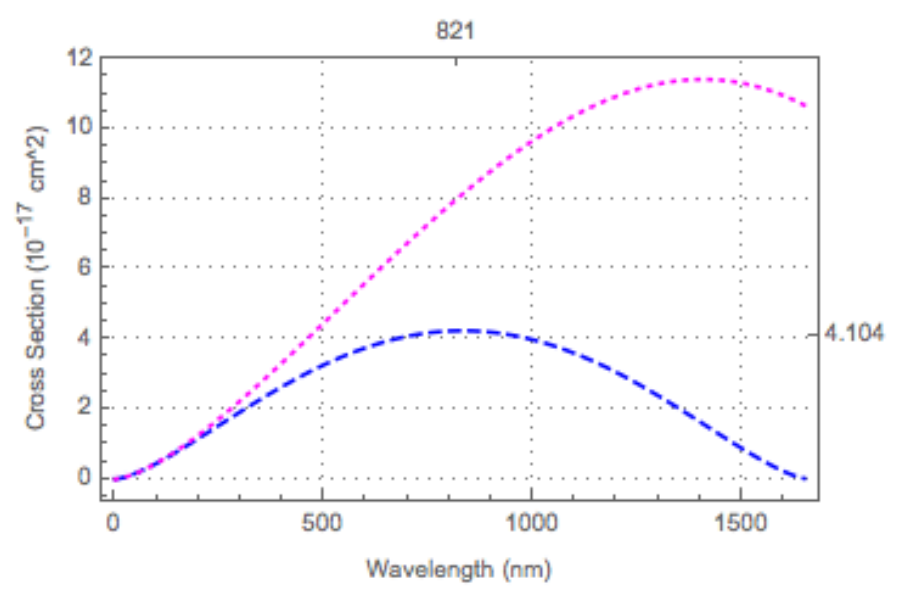

Figure 6.2: Cross Section vs. Wavelength for Photodetachment (PD). The dotted line is $z=0.5$. The dashed line is the dipole limit $z=0.01$.

and the strong field cross-section is

$$
\sigma_{P D}=\sum_{n=n_{0}}^{\infty} \frac{\hbar \omega}{\frac{1}{2} \epsilon_{0} c \omega^{2} A_{0}^{2}} \frac{1}{2 \pi} \mathcal{C}^{2} \frac{p_{0}}{m \hbar} p \Xi_{n}\left(k \alpha_{0},-\frac{z}{2}\right),
$$

where we have used the fact

$$
\left|\widetilde{\psi}_{e f f}(\mathbf{k})\right|^{2}=\frac{1}{\mathcal{V}} \frac{16 \pi^{2} C^{2}}{\left(k^{2}+\gamma^{2}\right)^{2}}
$$

The cross-section can be further simplified to

$$
\sigma_{P D}=\sum_{n=n_{0}}^{\infty} \alpha 2 \pi \frac{\hbar}{m \omega^{2}} \frac{1}{z}\left(\frac{1}{2 \pi} \mathcal{C}^{2} \frac{p_{0}}{m} k \Xi_{n}\left(k \alpha_{0},-\frac{z}{2}\right)\right) .
$$

The maximum cross-section for photodetachement at $z=0.5$ is

$$
\sigma_{P D}(z=0.5) \approx 10^{-16} \mathrm{~cm}^{2} .
$$


The rate coefficient is

$$
\alpha_{P D}(z=0.5)=c \sigma_{P D} \approx 10^{-6} \frac{\mathrm{cm}^{3}}{\mathrm{~s}} .
$$

\subsection{Strong Field Spontaneous Radiative Attachment (RA)}

Once again, we will use the principle of detailed balancing in order to obtain the cross-section for spontaneous radiative attachment,

$$
\sigma_{R A}=\frac{1}{2} \frac{\left(\frac{\hbar \omega}{c}\right)^{2}}{p^{2}} \sigma_{P D}
$$

For spontaneous radiative attachment there is no laser field present (i.e. $z \rightarrow 0$ ). Therefore,

$$
\sigma_{R A}=\lim _{z \rightarrow 0} \sum_{n=n_{0}}^{\infty} \frac{1}{2} \frac{\left(\frac{\hbar \omega}{c}\right)^{2}}{p^{2}} \alpha 2 \pi \frac{\hbar}{m \omega^{2}} \frac{1}{z}\left(\frac{1}{2 \pi} \mathcal{C}^{2} \frac{p_{0}}{m} k \Xi_{n}\left(k \alpha_{0},-\frac{z}{2}\right)\right)
$$

where we must use the condition that,

$$
n \hbar \omega=E_{k}+E_{B}+U_{p}(z=0)
$$

or

$$
n \hbar \omega=E_{k}+E_{B}
$$

The kinetic energy and bound state energy are both positive quantities. This equation always has a solution for $n \geq 1$, so that for the sum we start at

$$
n_{0}=1
$$


Solving for $\hbar \omega$, we find

$$
\hbar \omega=\frac{E_{k}+E_{B}}{n},
$$

where the lower limit on the sum prevents this quantity from having a singularity. Remembering that for radiative attachment we control the incoming electron energy.

$$
\sigma_{R A}=\lim _{z \rightarrow 0} \sum_{n=1}^{\infty} \frac{1}{2} \frac{1}{k^{2} c^{2}} \alpha 2 \pi \frac{\hbar}{m} \frac{1}{z}\left(\frac{1}{2 \pi} \mathcal{C}^{2} \frac{p_{0}}{m} k \Xi_{n}\left(k \alpha_{0},-\frac{z}{2}\right)\right)
$$

Evaluation of the limit leads to the same results as first order perturbation theory.

Since $z=0$ is indeed a weak field, we can also use the weak field limit. Thus,

$$
\begin{gathered}
\sigma_{R A}=\lim _{z \rightarrow 0} \frac{1}{2} \frac{1}{k^{2} c^{2}} \alpha 2 \pi \frac{\hbar}{m} \frac{1}{z}\left(\frac{1}{2 \pi} \mathcal{C}^{2} \frac{p_{0}}{m} k \int\left(\frac{\mathbf{k} \cdot \boldsymbol{\alpha}_{0}}{2}\right)^{2} d \Omega\right) \\
=1.213 \times 10^{-22} \mathrm{~cm}^{2} \frac{\left(\frac{p}{p_{0}}\right)}{\left(\frac{p}{p_{0}}\right)^{2}+1} .
\end{gathered}
$$

which is the same result as first order perturbation theory in Chapter 5.

\subsection{Strong Field Stimulated Attachment (SA)}

In this section, we analyze strong field stimulated attachment in hopes of increasing the rates of production. Starting with the T-matrix, we switch the final and initial state from that of photodetachment and again use integration by parts on the time integral. We find the T-matrix to be the same as the one for photodetachment,

$$
T_{f i}=\sum_{n=-\infty}^{\infty} 2 \pi i\left(E_{k}+E_{B}\right) \widetilde{\psi}_{e f f}(\mathbf{k})(-1)^{n} J_{n}\left(\mathbf{k} \cdot \boldsymbol{\alpha}_{0},-\frac{z}{2}\right) \delta\left(E_{k}+E_{B}+U_{p}-n \hbar \omega\right) .
$$


Next, we find the rate by integrating over the possible ejected photon frequencies,

$$
\begin{gathered}
\Gamma_{S A}=\sum_{f} \Gamma_{f i}=\int \Gamma_{f i} d^{3} n_{f}= \\
\int\left(\sum_{n=-\infty}^{\infty} \frac{2 \pi}{\hbar}\left(E_{k}+E_{B}\right)^{2}\left|\widetilde{\psi}_{e f f}(\mathbf{k})\right|^{2} J_{n}^{2}\left(\mathbf{k} \cdot \boldsymbol{\alpha}_{0},-\frac{z}{2}\right) \delta\left(E_{k}+E_{B}-\hbar \omega(n-z)\right)\right) \\
\times g_{S A} \frac{\mathcal{V}}{(2 \pi)^{3} \hbar^{3}}\left(\frac{\hbar \omega}{c}\right)^{2} \frac{\hbar}{c} d \omega d \Omega
\end{gathered}
$$

or

$$
\Gamma_{S A}=\sum_{n=n_{0}}^{\infty} \frac{2 \pi}{\hbar}\left(E_{k}+E_{B}\right)^{2}\left|\widetilde{\psi}_{e f f}(\mathbf{k})\right|^{2} g_{S A} \frac{\mathcal{V}}{(2 \pi)^{3} \hbar^{3}}\left(\frac{\hbar \omega}{c}\right)^{2} \frac{\hbar}{c} \int J_{n}^{2}\left(\mathbf{k} \cdot \boldsymbol{\alpha}_{0},-\frac{z}{2}\right) d \Omega
$$

At this point we can divide the strong field cross-section for stimulated attachment by the cross-section for radiative attachment. We again, find the principle of detailed balancing. That is,

$$
\sigma_{S A}=\mathcal{N} \frac{1}{2} \frac{\left(\frac{\hbar \omega}{c}\right)^{2}}{p^{2}} \sigma_{P D}
$$

This shows that the principle of detailed balancing is a general principle that does not depend on the assumptions of perturbation theory. Using the principle of detailed balancing, we find

$$
\sigma_{S A}=\sum_{n=n_{0}}^{\infty} \mathcal{N} \frac{1}{2} \frac{\left(\frac{\hbar \omega}{c}\right)^{2}}{p^{2}} \alpha 2 \pi \frac{\hbar}{m \omega^{2}} \frac{1}{z}\left(\frac{1}{2 \pi} \mathcal{C}^{2} \frac{p_{0}}{m} k \Xi_{n}\left(k \alpha_{0},-\frac{z}{2}\right) .\right)
$$

where we must use the condition that,

$$
n \hbar \omega=E_{k}+E_{B}+U_{p}(z)
$$




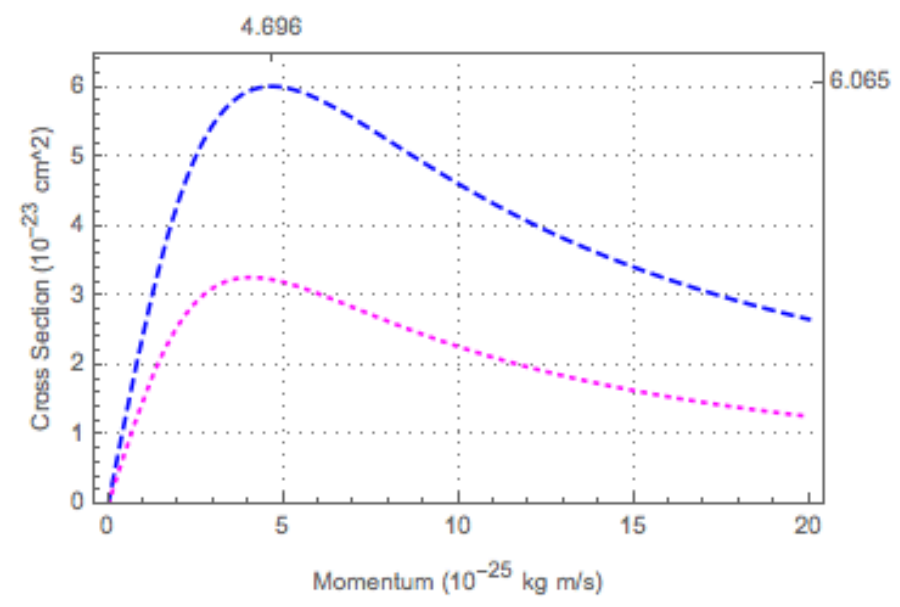

Figure 6.3: Cross Section (per photon) vs. Wavelength for Stimulated Attachment (SA). The dotted line is $z=0.5$. The dashed line is the dipole limit $z=0.01$.

or

$$
n \hbar \omega=E_{k}+E_{B}+\hbar \omega z
$$

Because the kinetic energy and bound state energy are both positive quantities, when $n=1$, the equation has no solutions for $z>1$. However, the equation does permit solutions for $n \geq 2$ for $1 \leq z \leq 2$. This sets the lower limit on where we start the sum.

$$
n_{0}=\lceil z\rceil
$$

This means that when $1 \leq z \leq 2$, the single photon interaction is forbidden by the strength of the ponderomotive energy of the laser field. Solving for $\hbar \omega$,

$$
\hbar \omega=\frac{E_{k}+E_{B}}{n-z}
$$

where the lower limit on the sum prevents this quantity from both becoming negative and as well as having a singularity.

Remember that for stimulated attachment we control the incoming electron en- 
ergy. For the, $n=1$, single photon attachment at $z=0.5$ we find the frequency of the laser where stimulated attachment occurs to be, using $p_{r m s}=1.943 \times 10^{-26} \mathrm{~kg} \frac{\mathrm{m}}{\mathrm{s}}$, $E_{B}=1.210 \times 10^{-19} \mathrm{~J}$

$$
\omega_{S A}(z=0.5)=2.298 \times 10^{15} s^{-1}
$$

This is twice the frequency as it was at perturbative intensity of $10^{10} \mathrm{~W} / \mathrm{cm}^{2}$, which was $\omega_{S A}=1.149 \times 10^{15} s^{-1}$. This shift in frequency is a direct result of strong field effects. Once again, our temperature is low; $p_{r m s}^{2} / 2 m$ is so small in comparion with $E_{B}$ that this value has not strayed too far from $\hbar \omega=E_{B} /(n-z)$. The wavelength of the laser where stimulated attachment occurs is

$$
\lambda_{S A}(z=0.5)=820 \mathrm{~nm}
$$

which is in the near infrared. We can see that the wavelength at which stimulated attachment occurs is now a function of the laser intensity! For $n=2$, there is also another resonance at $\omega_{S A}(z=0.5)=0.7660 \times 10^{15} s^{-1}$ or $\lambda_{S A}(z=0.5)=$ $2458 \mathrm{~nm}$. However, the two photon interaction is almost always less probable than the one photon interaction, with the exception being the case where the single photon interaction is forbidden by the high intensity of the laser field. Simplifying the crosssection a bit, we find

$$
\sigma_{S A}=\sum_{n=n_{0}}^{\infty} \mathcal{N} \frac{1}{2} \frac{1}{k^{2} c^{2}} \alpha 2 \pi \frac{\hbar}{m} \frac{1}{z}\left(\frac{1}{2 \pi} \mathcal{C}^{2} \frac{p_{0}}{m} k \Xi_{n}\left(k \alpha_{0},-\frac{z}{2}\right)\right)
$$

The number of photons is

$$
\mathcal{N}=\frac{I \mathcal{V}}{\hbar \omega c}=\frac{\left(10^{13} \mathrm{~W} / \mathrm{cm}^{2}\right)\left(1 \mathrm{~cm}^{3}\right)}{\left(2.424 \times 10^{-19} \mathrm{~J}\right)\left(2.998 \times 10^{\left.10 \frac{\mathrm{cm}}{\mathrm{s}}\right)}\right.}=1.4 \times 10^{21} \text { photons }
$$


Or we can convert the photons into an intensity by

$$
\mathcal{N}=\frac{I \mathcal{V}}{\hbar \omega c} \frac{I_{0}}{I_{0}}=\frac{I \mathcal{V}}{\hbar \omega c} \frac{1}{I_{0}} \frac{1}{\alpha} \frac{1}{2 \pi} m \omega^{3}=\frac{\mathcal{V}}{\hbar c} \frac{1}{\alpha} \frac{1}{2 \pi} m \omega^{2} z
$$

Using this conversion, the cross-section is

$$
\sigma_{S A}=\sum_{n=n_{0}}^{\infty} \frac{1}{2} \mathcal{V} \frac{\omega^{2}}{k^{2} c^{3}}\left(\frac{1}{2 \pi} \mathcal{C}^{2} \frac{p_{0}}{m} k \Xi_{n}\left(k \alpha_{0},-\frac{z}{2}\right)\right) .
$$

Evaluating the cross-section at $z=0.5$, we find

$$
\frac{\sigma_{S A}(z=0.5)}{\mathcal{N}}=5.004 \times 10^{-24} \mathrm{~cm}^{2}
$$

or

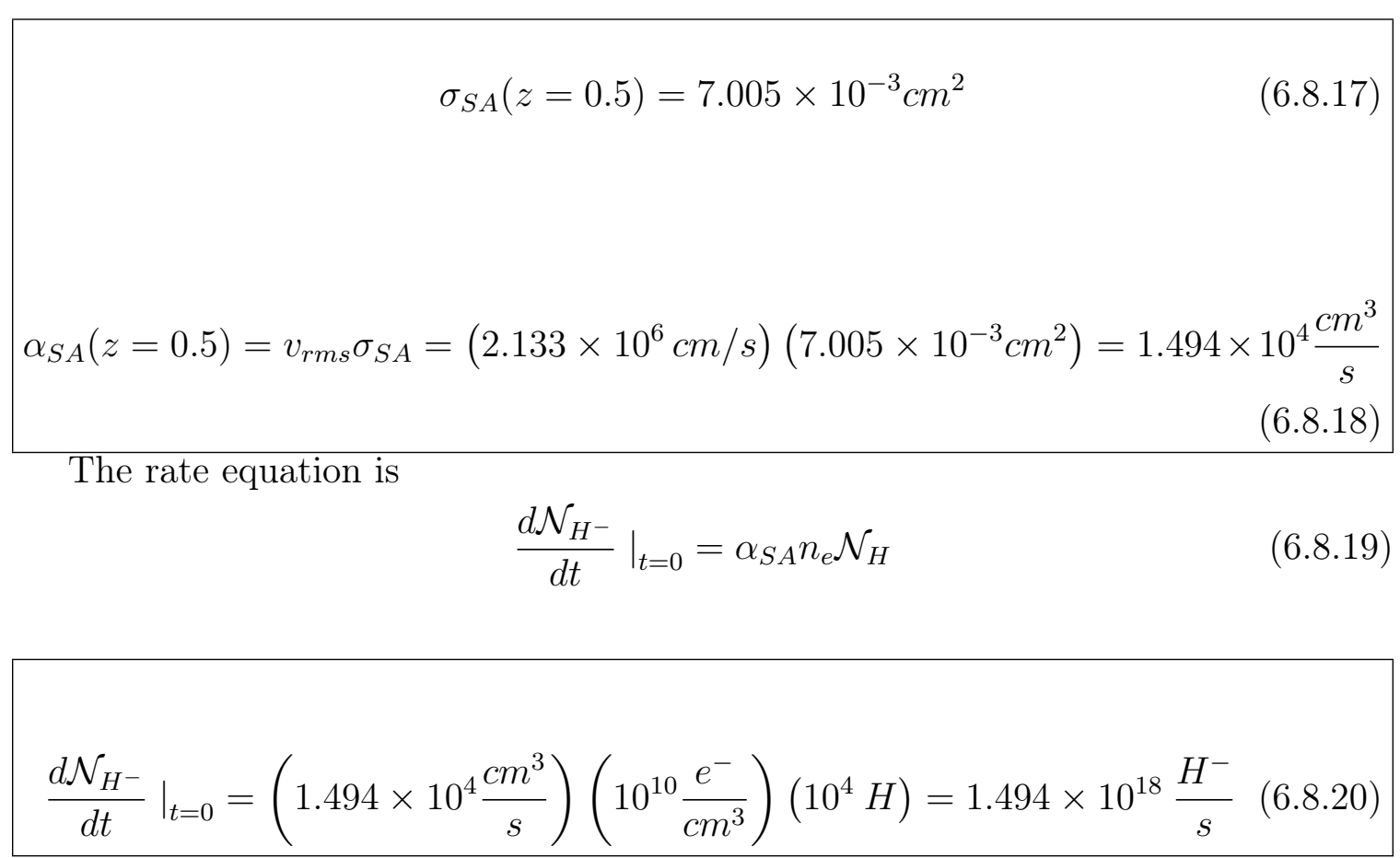

This is an even higher rate of production than we had from perturbation theory. Again, that rate is for continuous operation; a typical Ti-Sapphire pulse repetition 
rate is on the order of $70 \mathrm{MHz}$. That is a pulse repetition period of $15 \mathrm{~ns}$. This means the laser is only interacting with the hydrogen for about $5 \mu s$ out of each second of operation. This reduces the rate of production by a factor of $5 \times 10^{-6}$, giving a rate of about $7.5 \times 10^{12} \mathrm{~s}^{-1}$. This is still a high rate of production. In order to maintain this rate, we would need to actively remove the product. If we cannot remove the product, then the system will eventually reach equilibrium. At equilibrium, we find

$$
n_{H^{-}}=\frac{\beta_{S A}}{\alpha_{P D}} n_{e} n_{H}=\frac{\left(1.494 \times 10^{-17} \frac{\mathrm{cm}^{6}}{\mathrm{~s}}\right)}{\left(10^{-6} \frac{\mathrm{cm}^{3}}{\mathrm{~s}}\right)} n_{e} n_{H}
$$

or

$$
n_{H^{-}}=\left(10^{10} \frac{\mathrm{e}^{-}}{\mathrm{cm}^{3}}\right)\left(10^{4} \frac{\mathrm{H}}{\mathrm{cm}^{3}}\right)\left(1.494 \times 10^{-11} \mathrm{~cm}^{3}\right) \approx 1500 \frac{\mathrm{H}^{-}}{\mathrm{cm}^{3}}
$$

Again, this amount of antihydrogen ions is observable. 


\section{Chapter 7}

\section{Conclusion}

\subsection{Concluding Remarks}

In most antihydrogen experiments conducted to date, the positron plasmas are cold and dense enough that the antihydogen is produced in very weakly bound states via the three-body reaction $[12,16,21]$

$$
e^{+}+e^{+}+\bar{p} \rightarrow e^{+}+\bar{H} \text {. }
$$

But the probability of a further positron interacting to produce the antihydrogrion ion is small becuase the length scale governing the rate of reaction is the Thomson radius, $r_{c}=e^{2} / 4 \pi \epsilon_{0} k_{B} T$, typically of order $\mu m$. Production of small quantitites of $\bar{H}^{+}$via double charge exchange $[62,63,64,65,66,10]$

$$
\bar{p}+P s \rightarrow \bar{H}+e^{-},
$$

where $\bar{p}$ is an antiproton and the positronium (Ps) may be in an excited state, followed by the reaction

$$
\bar{H}+P s \rightarrow \bar{H}^{+}+e^{-},
$$


would yield less than one $\bar{H}^{+}$per 60 million $\bar{p}$ for a Ps cloud of density of around $7 \times 10^{11} \mathrm{~cm}^{-3}$, with the latter produced using an intense $\left(3 \times 10^{8} \mathrm{~s}^{-1}\right)$ positron beam accumulated for just over $100 \mathrm{~s}$. The low yield of this Ps route to $\bar{H}^{+}$has motivated the present study. Now that $\bar{H}$ can be trapped for extended periods, it will be feasible to exploit the positron-antihydrogen reaction to produce the $\bar{H}^{+}$ions and our yield of $1500 \frac{\bar{H}^{+}}{\mathrm{cm}^{3}}$ argues favorably for this new approach. The ions can then be cooled by sympathic cooling techniques for use in spectroscopic experiments or the gravitation test of antimatter preformed by the GBAR experiment. 


\section{Bibliography}

[1] "Tara Shears - Antimatter: Why the anti-world matters," Tara Shears, https://www.youtube.com/watch?v=0Fy6oiIRwJc, 2013.

[2] "Carl Anderson - Discovery of the Position," Alaina G. Levine. https://www.aps.org/programs/outreach/history/historicsites/anderson.cfm, 2009.

[3] "Evidence for the $2 \pi$ decay of the $K_{2}^{0}$ meson," J. H. Christenson, J. W. Cronin, V.L. Fitch, R. Turlay. Phys. Rev. Lett., (1964) 13138.

[4] "Observation of Direct CP Violation in $K_{S, L} \rightarrow \pi \pi$ Decys," A. Alavi-Harati etal. Phys. Rev. Lett. 83 (1999) 22.

[5] "A New measurement of direct $\mathrm{CP}$ vioation in two pion decays of the neutral kaon," B. Fanti et al. Phys. Lett. B. 465 (1990) 335.

[6] "Measurement of CP-vioating asymmetried in $B^{0}$ decays of CP Eigenstates," The BABAR Collaboration, B Aubet et al. Phys. Rev. Lett. 86 (2001) 2515.

[7] "Observation of Large CP Violation in the Neutral B Meson System," The Belle Collatbortaion, K. Abe et al. Phys. Rev. Lett. 87 (2001).

[8] "First Observation of CP Violatoin in the Decays of $B_{s}^{0}$ Mesons" LHCB Collaboration," R. Aaaij et al. Phys. Rev. Lett. 110 (2013). 
[9] "Antimatter matters," John Ellis. Nature, 424 (2003) 631.

[10] "Antihydrogen physics," M. Charlton et al. Phys. Rep (1994).

[11] "Production and detection of cold antihydrogen atoms," M. Amoretti et al. Nature 419 (2002) 456.

[12] "Driven Production of Cold Antihydrogen and the First Measured Distribution of Antihydrogen States," G. Gabrelse et al. Phys. Rev. Lett., 89 (2002) 213401.

[13] "Convinement of antihydrogen for 1,000 seconds," The ALPHA Collaboration. Nature Physics 7 (2011) 558.

[14] "CPT and Lorentz Tests in Hydrogen and Antihydrogen," R. Bluhm, V. A. Kostelechy, N. Russell. Phys. Res. Lett. 82 (1999) 2254.

[15] "Production and detection of cold antihydrogen atoms," M. Amoretti etal. Nature 419 (2002) 456.

[16] "Background-Free Observation of Cold Antihydrgen with Field-Ionization Analysis of its States," G. Gabrilese et al. Phys. Rev. Lett. 89 (2002) 213401.

[17] "Observation of the 1S-2S transtion in trapped antihydrogen," M. Ahmadi, et al. Nature Letter. 541, 26 (2017).

[18] "Positron trapping in an electrostatic well by inelastic collisions with nitrogen molecules," T. J. Murphy and C. M. Surko. Phys Rev. A. 46, 5696 (1992).

[19] "Thousandfold improvement in the measured antiproton mass," G. Gabrielse, etal. (ATRAP Collaboration) Phys. Rev. Lett. 631360 (1989).

[20] "New source of dense, cryogenic positron plasmas," L. V. Jorgensen, etal. (ATHENA Collaboration) Phys. Rev. Lett. 95025002 (2005). 
[21] "Evaporative cooling of antiprotons to cryogenic temperatures," G. B. Andresen, etal. (ALPHA Collaboration) Phys. Rev. Lett. 105013003 (2010).

[22] "Adiabatic cooling of antiprotons," G. Gabrielse, etal. (ATRAP Collaboration) Phys. Rev. Lett. 106073002 (2011).

[23] J. Walz and T. W. Hänsch. Gen. Relativ. Gravit. 36561 (2004)

[24] "On the production of the positive antihydrogen ion $\bar{H}^{+}$via radiative attachment," C. M. Keating, M Charlton and Jack C Straton. J. Phys. B. 47 (2014) 225202.

[25] "Producing the positive antihydrogen ion $\bar{H}^{+}$via radiative attachment," C. M. Keating, K. Y. Pak and Jack C Straton. J. Phys. B. 49 (2016) 074002.

[26] "Limits on Tunneling Theories of Strong-Field Ionization," H. R. Reiss. Phys. Rev. Lett. 101, 043002 (2008).

[27] "Atoms in Intense Laser Fields," C. J. Joachain, N. J. Kylstra, R. M. Potvliege. Cambridge University Press, 2012.

[28] "Dipole-approximation magnetic fields in strong laser beams," H. R. Reiss. Phys. Rev. A 63, 013409 (2000).

[29] "Introduction to the Theory of Laser-Atom Interactions," 2nd ed., Marvin H Mittleman. Plenum Press, 1993.

[30] "Historical roots of gauge invariance," J. D. Jackson, L. B. Okun. Rev. Mod. Phys. 73, 663 (2001).

[31] "From Lorenz to Coulomb and other explicit gauge transformations," J. D. Jackson. American Jour. Phys. 70, 917 (2002). 
[32] "Foundations of Strong-Field Physics," Howard R. Reiss. Lecutres on Ultrafast intense Laser Scinece 1, 94 (2010) 41.

[33] "Atoms and molecules in intense laser fields: gauge invariance of theory and models," A. d. Bandrauk, F Fillion-Gourdeau and D Lorin. J. Phys. B. vol. 46 $15(2013)$.

[34] "Gauge-equivalent intense-field approximations in velocity and length gauge to all orders," F. H. M. Faisel. Phys. Rev. A 75, 063412 (2012).

[35] "Generalized gauge-invariant formulations of the strong-field approximation," Yulian V. Vanne and Alejandro Saenz. Phys. Rev. A 79, 023421 (2009).

[36] "Analytic sources of inequivalence of the velocity gauge and length gauge," Rainer Dick. Phys. Rev. 94, 062118 (2016).

[37] "A manifestly gaueg-invariant description of interaction of atomic systems with strong fields in the dipole approximation," Adam Bechler and Marcin Sleczka. Phys. Lett. A. 375 1579-1582 (2011).

[38] "Stong-field ionization and gauge dependence of nonlocal potentials," T. C. Rensink and T. M. Antonsen Jr. Phys. Rev. A. 94, 063407 (2016).

[39] "Physics of Atoms and Molecules," 2nd ed., B.H. Bransden, C. J. Joachain. Pearson Education limited 2003.

[40] "Fine Structure of the Hydrogen Atom," W. E. Lamb. Phys. Rev. 85, 259 (1952).

[41] "Consequences of the postulate of a complete commuting set of observables in quantum electrodynamics," F. J. Belinfante. Phys. Rev. 128, 2832 (1962).

[42] D. M. Volkov, Z Phys. Phys. 94, 250 (1935). 
[43] "Laser-Enhanced Electron-Ion Caputre and Anithydrogen Formation," R. Neumann. Z. Phys. A - Atoms and Nuclei 313, 253-262 (1983).

[44] "Elementary Nuclear Theory," H. A. Bethe. John Wiley and Sons Inc. 1947.

[45] "The Photodetachment of the Negative Ion of Positronium," S. J. Ward, M. R. C McDowell, J. W. Humberston. Europhys. Lett., 1 (4), pp 167 (1986).

[46] "Free-Free Absorption Coefficient of the Nagative Hydrogen Ion," T. Ohmura and H. Ohmura. Astrophysical Journal, vol. 131, p. 8 (1959).

[47] "Continuous Absorption Due to Free-Free Transitons in Hydrogen," T. Ohmura and H. Ohmura. Phys. Rev. 121, 513 (1961).

[48] "Electron-Hydrogen Scattering at Low Energies," Phys. Rev. vol. 1881 (1959).

[49] "Bound state properties and astrophysical applicaitons of negatively charged hydrogen ions," A.M. Frolov, V.H. Smith Jr. The Journal of Chemical Physics (2003).

[50] "Photodetachment of the hydrogen and positronium negative ions," A.M. Frolov. Journal of Physics B. vol. 374 (2004).

[51] "Table of Integrals, Series, and Products," I. S. Gradshteyn, I. M. Ryzhik. Academic Press 7th ed. (2007).

[52] "On the Mathematical Description of Light Nuclei by the Method of Resonation Group Structure," John A. Wheeler. Phys. Rev. 53, 1107 (1937).

[53] "Effect of an intense electromagnetic filed on a weakly bound system," H. R. Reiss. Phys. Rev. A 22, 1786 (1980). 
[54] "Theoretical methods in quantum optics: S-matrix and Keldysh techniques for stong-field process," H. R. Reiss. Progress in Quantum Electronics 16, 1 (1992).

[55] "Relativistic strong-field photoionization," Journal of the Optical Society of America B 7, 4 p. 570 (1990).

[56] "Multiple absorbtion of laser phtons by atoms," F. H. M. Faisal. J. Phys. B. vol. $6,4(1973)$

[57] "Ionization in the field of a strong electromagnetic wave," L.V. Keldysh. Sov. Phys. JETP (1965).

[58] "Multiphoton detachment of electrons from negative ions," G. F. Gribakin and M. Yu Kuchiev Phys. Rev. A. vol. 555 (1997).

[59] "Effect of bound-state dressing in laser-assisted radiative recombination," Robert A. Muller etal. Phys. Rev. A. 92, 053426 (2015).

[60] "Reformulation of the strong-field approximation for light-matter interactions," A. Galstyan et al. Phys. Rev. A. 93, 023422 (2016).

[61] "On two-dimensional Bessel functions," H.J. Korsch, A. Klumpp and D. Watthaut. J. Phys. A. vol. 39, 48 (2006).

[62] "Advances in antihydrogen physics," Mike Charlton and Dirk Peter Van Der Werf. Science Progress Vol. 98, Iss. 1 (2015).

[63] "The GBAR experiment: gravitational behaviour of antihydrogen at rest," P. Perez and Y Sacquin. Class. Quantum Grav. 29184008 (2012).

[64] "Physics with antihydrogen," W. A. Bertsche et al. J. Phys. B. 48232001 (2015). 
[65] "Ultra-low energy antihydrogen," M. H. Holzscheiter and M Charlton. Reports on Progress in Physics. vol 631 (1999).

[66] " $\bar{H}^{+}$production from collisions between positronium and $\mathrm{keV}$ antiprotons for GBAR," P. Comimi etal. Hyperfine Interactions vol. 228 iss. 1-3, p. 159-165 (2014).

[67] "Low-energy electron-He scattering in a low-frequency laser field," N. J. Kylstra and C. J. Joachain. Phys. Rev. A 60, 2255 (1999). 


\section{Appendix A}

\section{Ultra Short Pulses}

With the more recent introduction of laser systems that can obtain attosecond pulses, pulse times (i.e $\sim 100$ attoseconds) could be of the order of a few optical periods (i.e $\sim 1$ femtoseconds). In the case of attosecond lasers, the pulse time must be dealt with explicitly. In the dipole approximation

$$
\mathbf{A}(t)=A(t) \cos (\omega t) \hat{\boldsymbol{\epsilon}}
$$

where $A(t)$ is the pulse shape function (i.e. Gaussian, sech or similar function) and is the Fourier pair of the function $A\left(\omega^{\prime}\right)$. In the case of non-monochromatic light with pulse of about ten optical cycles or more, the wave packet can be modeled by a frequency distribution function such as a Gaussian

$$
\begin{aligned}
A\left(\omega^{\prime}\right)=\left\langle\omega^{\prime} \mid A\right\rangle & =A_{0} 2 \pi \frac{1}{\sqrt{2 \pi} \Delta \omega} \exp \left(-\frac{1}{2 \Delta \omega^{2}}\left(\omega^{\prime}-\omega\right)^{2}\right) \\
& =A_{0} 2 \pi \frac{1}{\sqrt{2 \pi}} 2 \Delta t \exp \left(-\frac{(2 \Delta t)^{2}}{2}\left(\omega^{\prime}-\omega\right)^{2}\right) .
\end{aligned}
$$

We find that the pulse shape function Fourier pair to be

$$
\begin{aligned}
A(t)=\langle t \mid A\rangle & =A_{0} \exp \left(-\frac{\Delta \omega^{2}}{2} t^{2}\right) \\
& =A_{0} \exp \left(-\frac{1}{2(2 \Delta t)^{2}} t^{2}\right) \\
& =A_{0} \exp \left(-\left(\frac{t}{\tau / 2}\right)^{2}\right),
\end{aligned}
$$


where $\tau=4 \sqrt{2} \Delta t$ is the spread in time of the pulse (pulse width), and the second line has used the Fourier uncertainty principles. We note that in the limit as the spread in the frequency goes to zero $A\left(\omega^{\prime}\right)$ becomes

$$
\lim _{\Delta \omega \rightarrow 0} A\left(\omega^{\prime}\right)=A_{0} 2 \pi \lim _{\Delta \omega \rightarrow 0} \frac{1}{\sqrt{2 \pi} \Delta \omega} \exp \left(-\frac{1}{2 \Delta \omega^{2}}\left(\omega^{\prime}-\omega\right)^{2}\right)=A_{0} 2 \pi \delta\left(\omega^{\prime}-\omega\right),
$$

where $\delta(x)$ is the Dirac delta function. In contrast, the same limit for the pulse shape function $A(t)$ is

$$
\lim _{\Delta \omega \rightarrow 0} A(t)=A_{0} \lim _{\Delta \omega \rightarrow 0} \exp \left(-\frac{(2 \Delta \omega)^{2}}{2} t^{2}\right)=A_{0}
$$

leading directly to a plane wave solution with frequency $\omega$. Now we need to be careful in the other limit where the pulses of light are ultra short pulses. If one takes the limit as the frequency become very large for the Gausian wave packet vector potential, one will find

$$
\lim _{\Delta \omega \rightarrow \infty} A(t)=A_{0} \lim _{\Delta \omega \rightarrow \infty} \exp \left(-\frac{(2 \Delta \omega)^{2}}{2} t^{2}\right)=\left\{\begin{array}{cc}
A_{0} & t=0 \\
0 & t \neq 0
\end{array}\right.
$$

This is not a Dirac delta function. There is an asymmetry between time and frequency. We note that although we can have a delta function frequency distribution, the time interaction is never a delta function because the interaction strength never exceeds $A_{0}$. This same asymmetry comes into play with the wavenumber distribution and spacial spread in the wavepacket. That is, the wavenumber distribution can be a delta function, whereas the spacial strength must never exceed $A_{0}$ and will never be a delta function.

In order to better understand the time dependence of the vector potential, we 
remind ourselves that the transverse electric field is related to the time derivative of the vector potential,

$$
\mathbf{E}_{\text {trans }}=-\frac{\partial \mathbf{A}}{\partial t} .
$$

We would like the transverse electric field to be an ultra short Gausian pulse

$$
\mathbf{E}_{\text {trans }}(t)=E_{0} \exp \left(-\frac{1}{2(2 \Delta t)^{2}} t^{2}\right) \sin (\omega t) \hat{\boldsymbol{\epsilon}},
$$

where $E_{0}=A_{0} \omega$. We find the vector potential to be

$$
\begin{gathered}
\mathbf{A}(t)=i A_{0} \sqrt{\frac{\pi}{2}} \omega \Delta t \exp \left(-\frac{(2 \Delta t)^{2}}{2} \omega^{2}\right) \\
\times\left[\operatorname{erf}\left(-\frac{i(2 \omega \Delta t)^{2}+\omega t}{2 \sqrt{2} \omega \Delta t}\right)+\operatorname{erf}\left(-\frac{i(2 \omega \Delta t)^{2}-\omega t}{2 \sqrt{2} \omega \Delta t}\right)\right] \hat{\boldsymbol{\epsilon}} .
\end{gathered}
$$

We note that for the condition that the pulse width is much greater than optical period $\tau \gg T$ (i.e $\omega \Delta t \gg 1$ or $E \Delta t \gg \hbar$ ),

$$
\mathbf{A}(t) \approx A_{0} \exp \left(-\frac{1}{2(2 \Delta t)^{2}} t^{2}\right)[\cos (\omega t)] \hat{\boldsymbol{\epsilon}}
$$

This is the same equation, the Gaussian wave packet, with which we started this analysis for the vector potential. However, now we can clearly see that the vector potential is only a Gaussian wave packet in the approximation of long pulse times. Thus, we see the time-energy uncertainty principle is a condition from which we can justify the use of a Gaussian wave packet vector potential.

Now, let us examine the case when the interaction time is much shorter than the 
optical period, $\tau \ll T$ (i.e $\omega \Delta t \ll 1$ or $E \Delta t \ll \hbar$ ). We find

$$
\mathbf{A}(t) \approx A_{0} \sqrt{\frac{\pi}{2}} \omega \Delta t \exp \left(-\frac{(2 \Delta t)^{2}}{2} \omega^{2}\right)\left[\sqrt{10} \omega \Delta t \exp \left(-\frac{1}{2(2 \Delta t)^{2}} t^{2}\right) \cos (\omega t)\right] \hat{\boldsymbol{\epsilon}}
$$

Or more simply,

$$
\mathbf{A}(t) \approx A_{0} \sqrt{\frac{\pi}{2}} \omega \Delta t\left[\sqrt{10} \omega \Delta t \exp \left(-\frac{1}{2(2 \Delta t)^{2}} t^{2}\right)\right] \hat{\boldsymbol{\epsilon}},
$$

where the $\cos (\omega t)$ behavior is roughly one over the pulse width because the optical period is large compared to the Gaussian pulse. This function clearly goes to zero for ultra short pulse times (even at $t=0$ !). This corresponds to a transverse electric field

$$
\begin{aligned}
\mathbf{E}_{\text {trans }} & \approx E_{0} \sqrt{\frac{\pi}{2}} \sqrt{10} \frac{\omega t}{4} \exp \left(-\frac{1}{2(2 \Delta t)^{2}} t^{2}\right) \hat{\boldsymbol{\epsilon}} \\
& \approx \quad E_{0} \exp \left(-\frac{1}{2(2 \Delta t)^{2}} t^{2}\right) \omega t \hat{\boldsymbol{\epsilon}} .
\end{aligned}
$$

We note that $\sqrt{\frac{\pi}{2}} \sqrt{10} \approx 4$ and $\sin (\omega t) \approx \omega t$ giving good agreement with the Gaussian wave packet we started with. The frequency distribution function for the vector potential is

$$
\begin{aligned}
A\left(\omega^{\prime}\right) & = & A_{0} \sqrt{\frac{\pi}{2}} \sqrt{10} \omega^{2} \Delta t^{2} \exp \left(-\frac{(2 \Delta t)^{2}}{2} \omega^{2}\right)\left[2 \pi \frac{1}{\sqrt{2 \pi}} 2 \Delta t \exp \left(-\frac{(2 \Delta t)^{2}}{2}\left(\omega^{\prime}-\omega\right)^{2}\right)\right] \\
& = & A_{0} 2 \pi \sqrt{10} \omega^{2} \Delta t^{3} \exp \left(-\frac{(2 \Delta t)^{2}}{2} \omega^{2}\right) \exp \left(-\frac{(2 \Delta t)^{2}}{2}\left(\omega^{\prime}-\omega\right)^{2}\right) .
\end{aligned}
$$

Now we cleary see the ultra short pulse limit. As the pulse gets shorter the frequency will spread out and the amplitude decreases to zero. The essence of the time-energy uncertainty principle here is that if the pulse time is of the order of the optical period then the interaction is no longer periodic. It basically has no frequencies in the Fourier distribution. In fact, attosecond lasers use X-ray frequency radiation, and are essentially "white" light ("white" meaning a wide range of frequencies.) 
Now, we look at the case where the electric field is turned on very fast. In this case, the electric field is

$$
\mathbf{E}_{\text {trans }}(t)=E_{0} \operatorname{rect}\left(\frac{t}{\tau}\right) \sin (\omega t) \hat{\boldsymbol{\epsilon}}
$$

where $\operatorname{rect}(x)$ is the rectangular (unit box) function.

$$
\operatorname{rect}(x)=\left\{\begin{array}{cc}
0 & |x|>\frac{1}{2} \\
\frac{1}{2} & |x|=\frac{1}{2} \\
1 & |x|<\frac{1}{2}
\end{array}\right.
$$

Integrating the electric field, we find the vector potential to be

$$
\mathbf{A}(t)=A_{0} \operatorname{rect}\left(\frac{t}{\tau}\right)\left(\cos (\omega t)-\cos \left(\omega \frac{\tau}{2}\right)\right) \hat{\boldsymbol{\epsilon}}
$$

We can check this by taking the derivative

$$
-\frac{\partial \mathbf{A}}{\partial t}=A_{0}\left[\omega \operatorname{rect}\left(\frac{t}{\tau}\right) \sin (\omega t)-\left(\delta\left(t+\frac{\tau}{2}\right)-\delta\left(t-\frac{\tau}{2}\right)\right)\left(\cos (\omega t)-\cos \left(\omega \frac{\tau}{2}\right)\right)\right] \hat{\boldsymbol{\epsilon}}
$$

Then the second term is indeterminant at the end point $t= \pm \tau / 2$ because the infinite delta functions are multiplying a term equal to zero. We can approximate rect $\left(\frac{t}{\tau}\right)$ by the function

$$
\operatorname{rect}\left(\frac{t}{\tau}\right) \approx \frac{1}{1+\left(\frac{t}{\tau / 2}\right)^{2 n}}
$$

where $n$ is a large integer. A closer look at the delta functions in the second term shows

$$
\left(t+\frac{\tau}{2}\right)-\delta\left(t-\frac{\tau}{2}\right) \approx-\frac{\frac{2 n}{t}\left(\frac{t}{\tau / 2}\right)^{2 n}}{\left(1+\left(\frac{t}{\tau / 2}\right)^{2 n}\right)^{2}} .
$$


This expression is $\mp \frac{n}{\tau}$ finite in the vicinity of $t= \pm \tau / 2$ for all finite $n$. This implies that the whole second term is finite and is equal to zero (i.e. finitexzero=zero). This gives us the correct expression for the electric field. The Fourier transform of the $\operatorname{rect}\left(\frac{t}{\tau}\right)$ function is

$$
A\left(\omega^{\prime}\right)=A_{0} \frac{\sin \left(\left(\omega^{\prime}-\omega\right) \tau / 2\right)}{\left(\omega^{\prime}-\omega\right) / 2} .
$$

For very long pulse times compared to optical period, the frequency distribution function behaves like

$$
A\left(\omega^{\prime}\right)=A_{0} 2 \pi \delta\left(\omega^{\prime}-\omega\right),
$$

as expected. The proof of this fact is rather simple

$$
\begin{gathered}
\lim _{\tau \rightarrow \infty} \frac{\sin \left(\left(\omega^{\prime}-\omega\right) \tau / 2\right)}{\left(\omega^{\prime}-\omega\right) / 2}=\lim _{\tau \rightarrow \infty} \frac{e^{i\left(\omega^{\prime}-\omega\right) \tau / 2}-e^{-i\left(\omega^{\prime}-\omega\right) \tau / 2}}{i\left(\omega^{\prime}-\omega\right)} \\
=\lim _{\tau \rightarrow \infty} \int_{-\tau / 2}^{\tau / 2} e^{i\left(\omega^{\prime}-\omega\right) t} d t=2 \pi \delta\left(\omega^{\prime}-\omega\right) .
\end{gathered}
$$

Again, for very short pulse times the frequency distribution function spreads out and the amplitude decreases to zero. 


\section{Appendix B}

\section{Perturbation with Pulse Shape Function}

Consider a system subjected to a periodic perturbation

$$
H^{\prime}(t)=H^{\prime} e^{-i \omega t}
$$

where $H^{\prime}(t)$ now has explicit periodic dependence on time and $H^{\prime}$ is the time independent part. It is important to remember that the perturbation is turned off at some time in the past as well as in the future. This could be included explicitly by a pulse shape function (perhaps $f(t)=\exp \left(-t^{2} /(\tau / 2)^{2}\right)$ or $f(t)=\operatorname{rect}(t / \tau)$ ), where perturbation time $\tau$ is large compared to the optical period). These functions are zero at times in the far past as well as zero at times in the far future. Though the pulse shape function is mathematically more rigorous, it is also more tedious. Plugging the periodic perturbation in and noticing that the final state is not the same as the initial state (i.e. $\left.\delta_{f i}=0\right)$, we find

$$
d_{f}(t)=-\frac{i}{\hbar} \int \psi_{f}^{(0) *}(\mathbf{r}) H^{\prime} \psi_{i}^{(0)}(\mathbf{r}) d^{3} x \int_{t_{0}}^{t_{f}} e^{i\left(\omega_{f i}-\omega\right) t} d t
$$


Let us have the perturbation start at $t_{0}=-\tau / 2$ and end at $t_{f}=\tau / 2$ for symmetry. Now, using the pulse shape function, $f(t)=\operatorname{rect}(t / \tau)$,

$$
\begin{aligned}
d_{f}(\tau) & =-\frac{i}{\hbar} \int \psi_{f}^{(0) *}(\mathbf{r}) H^{\prime} \psi_{i}^{(0)}(\mathbf{r}) d^{3} x \int_{-\infty}^{\infty} \operatorname{rect}(t / \tau) e^{i\left(\omega_{f i}-\omega\right) t} d t \\
& =\quad-\frac{i}{\hbar} \int \psi_{f}^{(0) *}(\mathbf{r}) H^{\prime} \psi_{i}^{(0)}(\mathbf{r}) d^{3} x\left[\frac{\sin \left(\left(\omega_{f i}-\omega\right) \tau / 2\right)}{\left(\omega_{f i}-\omega\right) / 2}\right] .
\end{aligned}
$$

Notice that if $\omega \neq \omega_{f i}$ for finite perturbation times the function $\sin \left(\left(\omega^{\prime}-\omega\right) \tau / 2\right)$ oscillates between zero and one. As perturbation time $\tau$ gets very large,

$$
\lim _{\tau \rightarrow \infty} \frac{\sin \left(\left(\omega_{f i}-\omega\right) \tau / 2\right)}{\left(\omega_{f i}-\omega\right) / 2}=\lim _{\tau \rightarrow \infty} \int_{-\tau / 2}^{\tau / 2} e^{i\left(\omega_{f i}-\omega\right) t} d t=2 \pi \delta\left(\omega_{f i}-\omega\right) .
$$

This gives

$$
d_{f}(\tau \rightarrow \infty)=-2 \pi \frac{i}{\hbar} \int \psi_{f}^{(0) *}(\mathbf{r}) H^{\prime} \psi_{i}^{(0)}(\mathbf{r}) d^{3} x \delta\left(\omega_{f i}-\omega\right)
$$

The transition probability is defined as

$$
P_{f i}(\tau) \equiv\left|\int \Psi^{*}(\mathbf{r}, \tau) \Psi(\mathbf{r},-\infty) d^{3} x\right|^{2}=\left|d_{f}(\tau)\right|^{2}
$$

For finite perturbation times, we find

$$
P_{f i}(\tau)=\frac{1}{\hbar^{2}}\left|\int \psi_{f}^{(0) *}(\mathbf{r}) H^{\prime} \psi_{i}^{(0)}(\mathbf{r}) d^{3} x\right|^{2}\left[\frac{\sin \left(\left(\omega_{f i}-\omega\right) \tau / 2\right)}{\left(\omega_{f i}-\omega\right) / 2}\right]^{2}
$$

Again, notice that when $\omega \neq \omega_{f i}$ for finite perturbation times the function $\sin ^{2}\left(\left(\omega^{\prime}-\omega\right) \tau / 2\right)$ oscillates between zero and one. One might try setting up the system such that the perturbation time is $\tau_{n}=2 \pi n /\left(\omega_{f i}-\omega\right)$, thereby catching the system in a transition. Now that we know what happens when $\omega \neq \omega_{f i}$, we ask what 
happens if $\omega=\omega_{f i}$. Looking at the limit as $\omega_{f i}-\omega$ goes to zero, we see that

$$
\lim _{\omega_{f i}-\omega \rightarrow 0} \frac{\sin \left(\left(\omega_{f i}-\omega\right) \tau / 2\right)}{\left(\omega_{f i}-\omega\right) / 2}=\tau,
$$

for all finite perturbation times. Interesting enough, the probability of a transition increases with the pertubation time without bound! But we already knew this from

$$
\lim _{\tau \rightarrow \infty} \frac{\sin \left(\left(\omega_{f i}-\omega\right) \tau / 2\right)}{\left(\omega_{f i}-\omega\right) / 2}=2 \pi \delta\left(\omega_{f i}-\omega\right)
$$

This seems like a paradox: after all, probabilities are never greater than one. However, what we really have is the probability of a transition from an initial state to the final state with a photon of exactly the right energy to get into the final state. This implies we know the final state energy exactly but in real life we have a range of energy that we are interested in. That means we would need to integrate over a range of frequencies (more precisely, the density of states labeled by their energies or frequencies) in order to get the probability of a transition between the chosen range of frequencies. This is the same as the paradox about the probability of getting exactly some number from a continuous distribution, perhaps a Gaussian distribution, in probability theory. The probability of getting exactly some number is of course zero! 


\section{Appendix C}

\section{Effective Range Theory}

Recall the unperturbed Hamiltonian,

$$
H_{0}=\frac{\mathbf{p}_{1}^{2}}{2 m}+\frac{\mathbf{p}_{2}^{2}}{2 m}-\frac{e^{2}}{\left(4 \pi \epsilon_{0}\right) r_{1}}-\frac{e^{2}}{\left(4 \pi \epsilon_{0}\right) r_{2}}+\frac{e^{2}}{\left(4 \pi \epsilon_{0}\right) r_{12}}
$$

Solutions to the unperturbed Hamiltonian are simply the wavefunctions $\psi_{n}^{(0)}\left(\mathbf{r}_{1}, \mathbf{r}_{2}\right)$ and can be found by time-independent perturbation theory, the variational principle, or other approximation methods. Keating, Pak and Straton did their analysis using highly accurate variational wavefunctions [25]. Herein we have used the so called effective range theory,

$$
H_{0}=\frac{\mathbf{p}_{1}^{2}}{2 m}+\frac{\mathbf{p}_{2}^{2}}{2 m}-\frac{e^{2}}{\left(4 \pi \epsilon_{0}\right) r_{1}}-e V_{e f f}\left(r_{2}\right)
$$

where

$$
V_{e f f}\left(r_{2}\right)=\left\{\begin{array}{cc}
V_{e f f} & r_{2}<a \\
0 & r_{2}>a
\end{array},\right.
$$

where $V_{\text {eff }}$ is a negative number. The second electron is effectively in a finite spherical well with a radius $a$. This is of course an approximation but one that has been shown to be well within the accuracy needed here. With the effective range approximation, 
the two electron wavefunctions become separable. The first electron Hamiltonian is

$$
H_{0}\left(\mathbf{r}_{1}\right)=\frac{\mathbf{p}_{1}^{2}}{2 m}-\frac{e^{2}}{\left(4 \pi \epsilon_{0}\right) r_{1}} .
$$

This is simply the Hamiltonian for the Hydrogen atom. The solutions are well known. The second electron Hamiltonian is

$$
H_{0}\left(\mathbf{r}_{2}\right)=\frac{\mathbf{p}_{2}^{2}}{2 m}-e V_{e f f}\left(r_{2}\right) .
$$

This is the Hamiltonian of a particle in a finite spherical well. Its solutions are also well known. We see that the separability of the wavefunction has alleviated a fair amount of complexity from our analysis. The ground state wavefunction for the Hydrogen atom is

$$
\psi_{1 s}(\mathbf{r})=\frac{1}{\sqrt{\pi a_{0}^{3}}} e^{-\frac{r}{a_{0}}} .
$$

The derivation of the Hydrogenic wavefunction can be found in any undergraduate textbook. The ground state wavefunction for the finite spherical well is

$$
\psi_{e f f}(\mathbf{r})=\left\{\begin{array}{cc}
\frac{A}{r} \sin \left(k^{\prime} r\right) & r<a \\
\frac{C}{r} \exp (-\gamma r) & r>a
\end{array} .\right.
$$

We will derive the solution for the finite spherical well since it is not covered as much in textbooks. We start with the Schrödinger equation for the finite spherical well

$$
-\frac{\hbar^{2}}{2 m} \nabla^{2} \psi_{e f f}-e V_{e f f}(r) \psi_{e f f}=-E_{B} \psi_{e f f} .
$$


The next step is to separate the angular and radial parts. Therefore, we look for solutions that separate the wavefunction into the product

$$
\psi_{e f f}(r, \theta, \phi)=R(r) Y(\theta, \phi) .
$$

The normalized solutions to the angular part are well known spherical harmonics $Y_{l m}(\theta, \phi)$. We are interested in the ground state, so we will need

$$
Y_{00}(\theta, \phi)=\frac{1}{\sqrt{4 \pi}} .
$$

The radial equation has the form

$$
\begin{array}{cc}
\frac{d}{d r}\left(r^{2} \frac{d}{d r} R\right)+\frac{2 m}{\hbar^{2}}\left[e V_{e f f}-E_{B}\right] R=l(l+1) R & r<a \\
\frac{d}{d r}\left(r^{2} \frac{d}{d r} R\right)+\frac{2 m}{\hbar^{2}}\left[0-E_{B}\right] R=l(l+1) R & r>a .
\end{array}
$$

This equation is simplified by the changing to the function

$$
u(r)=r R(r) .
$$

This gives us the radial equation

$$
\begin{array}{rr}
-\frac{\hbar^{2}}{2 m} \frac{d^{2} u}{d r^{2}}+\left[-e V_{e f f}+\frac{\hbar^{2}}{2 m} \frac{l(l+1)}{r^{2}}\right] u=-E_{B} u & r<a \\
-\frac{\hbar^{2}}{2 m} \frac{d^{2} u}{d r^{2}}+\left[0+\frac{\hbar^{2}}{2 m} \frac{l(l+1)}{r^{2}}\right] u=-E_{B} u . & r>a .
\end{array}
$$

With our potential, the inside $a$ equation has sinusoidal solutions and the outside $a$ equation has exponential solutions. The wavefunction and its derivative should be continuous at $r=0$ and must not diverge faster than $\mathrm{r}$ as $r \rightarrow \infty$. The general solution for $R(r)$, that satisfies our boundary conditions of finite at both $r=0$ and 
$r \rightarrow \infty$, are the well known spherical Bessel functions inside $a$ and exponential outside $a$

$$
R_{n l}(r)=\left\{\begin{array}{cc}
A j_{l}\left(k_{n}^{\prime} r\right) & r<a \\
B \exp (-\gamma r) & r>a
\end{array} .\right.
$$

We are interested in the ground state wavefunction, and therefore we will need

$$
R_{00}(r)=A j_{0}\left(k^{\prime} r\right),
$$

where

$$
\begin{gathered}
j_{0}(x)=\frac{\sin (x)}{x}, \\
k^{\prime}=\frac{\sqrt{2 m\left(e V_{e f f}-E_{B}\right)}}{\hbar},
\end{gathered}
$$

in which we have dropped the $n$ subscript on the $k^{\prime}$, and

$$
\gamma=\frac{\sqrt{2 m E_{B}}}{\hbar} .
$$

To summarize, the finite square well wavefunction is

$$
\psi_{e f f}(\mathbf{r})=\left\{\begin{array}{cc}
\frac{A}{r} \sin \left(k^{\prime} r\right) & r<a \\
\frac{C}{r} \exp (-\gamma r) & r>a
\end{array},\right.
$$

where we have absorbed the a $k^{\prime}$ into the definition of $A$. The wavefunction and its derivative should also be continuous at $r=a$. This condition gives the equations

$$
\left\{\begin{array}{c}
\frac{C}{a} \exp (-\gamma a)=\frac{A}{a} \sin \left(k^{\prime} a\right) \\
-\gamma \frac{C}{a} \exp (-\gamma a)-\frac{C}{a^{2}} \exp (-\gamma a)=k^{\prime} \frac{A}{a} \cos \left(k^{\prime} a\right)-\frac{A}{a^{2}} \sin \left(k^{\prime} a\right) .
\end{array}\right.
$$




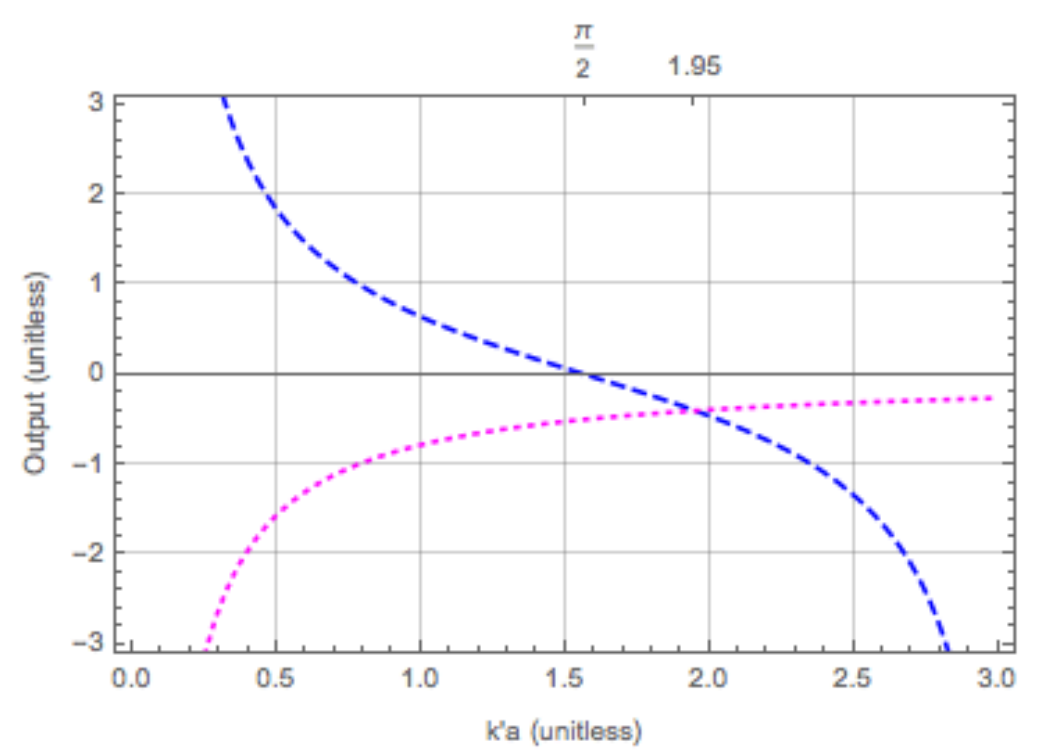

Figure C.1: Transcendental Equation: Dashed line is $\cot \left(k^{\prime} a\right)$. Dotted line is $-(0.777) / k^{\prime} a$. Notice, the two functions cross at 1.95 . This is close to the place where $\cot \left(k^{\prime} a\right)=0$, which is $\pi / 2$.

Dividing these two equations, we find

$$
-\gamma-\frac{1}{a}=\frac{k^{\prime} \cos \left(k^{\prime} a\right)}{\sin \left(k^{\prime} a\right)}-\frac{1}{a} .
$$

or simply

$$
\cot \left(k^{\prime} a\right)=-\frac{\gamma}{k^{\prime}}=-\frac{\gamma a}{k^{\prime} a},
$$

which conveniently does not involve $A$ and $C$. For a given $\gamma=\frac{\sqrt{2 m E_{B}}}{\hbar}$ and given $a$ this equation sets $k$ '. The equation happens to be transcendental. It has to be solved either graphically or numerically. However, if we know $k^{\prime}>\gamma$, then $-\frac{\gamma}{k^{\prime}}$ is a small number and we can say $\cot \left(k^{\prime} a\right) \approx 0$. This implies $k^{\prime} a \gtrsim \pi / 2$.

Now we will turn our attention to normalization of the wavefunction.

$$
\int_{\Omega} \int_{0}^{a} \frac{A^{2}}{r^{2}} \sin ^{2}\left(k^{\prime} r\right) r^{2} d r d \Omega+\int_{\Omega} \int_{a}^{\infty} \frac{C^{2}}{r^{2}} \exp (-2 \gamma r) r^{2} d r d \Omega=1 .
$$


Evaluating the integrals, we find

$$
4 \pi A^{2}\left[\frac{a}{2}-\frac{\sin \left(2 k^{\prime} a\right)}{4 k^{\prime}}\right]+4 \pi C^{2} \frac{1}{2 \gamma} \exp (-2 \gamma a)=1 .
$$

Using the fact that wavefunctions must be continuous at $r=a$,

$$
A=C \frac{\exp (-\gamma a)}{\sin \left(k^{\prime} a\right)} .
$$

We simplify

$$
\begin{gathered}
4 \pi C^{2} \frac{\exp (-2 \gamma a)}{\sin ^{2}\left(k^{\prime} a\right)}\left[\frac{a}{2}-\frac{\sin \left(2 k^{\prime} a\right)}{4 k^{\prime}}\right]+4 \pi C^{2} \frac{1}{2 \gamma} \exp (-2 \gamma a)=1 \\
4 \pi C^{2} \frac{1}{2 \gamma} \exp (-2 \gamma a)\left[\frac{2 \gamma}{\sin ^{2}\left(k^{\prime} a\right)}\left[\frac{a}{2}-\frac{\sin \left(2 k^{\prime} a\right)}{4 k^{\prime}}\right]+1\right]=1 \\
C^{2}\left[\frac{\gamma a}{\sin ^{2}\left(k^{\prime} a\right)}\left[1-\frac{\sin \left(2 k^{\prime} a\right)}{2 k^{\prime} a}\right]+1\right]=\frac{\gamma}{2 \pi} e^{2 \gamma a}
\end{gathered}
$$

to obtain

$$
C=\frac{\sqrt{\gamma / 2 \pi} e^{\gamma a}}{\sqrt{1+\frac{\gamma a}{\sin ^{2}\left(k^{\prime} a\right)}\left[1-\frac{\sin \left(2 k^{\prime} a\right)}{2 k^{\prime} a}\right]}} .
$$

Besides the finite spherical well, other types of effective range potentials have also been used. Examples include the finite harmonic well,

$$
V_{e f f}(r)=\left\{\begin{array}{cc}
V_{e f f}\left(\frac{r}{a}\right)^{2}-V_{e f f} & r<a \\
0 & r>a
\end{array}\right.
$$


and the constant force finite triangle potential,

$$
V_{e f f}(r)=\left\{\begin{array}{cc}
V_{e f f} \frac{r}{a}-V_{e f f} & r<a \\
0 & r>a .
\end{array}\right.
$$

These potentials increase the complexity of the analysis yet give very similar results. 


\section{Appendix D}

\section{Derivation of the Function $\Xi_{n}\left(k \alpha_{0},-\frac{z}{2}\right)$}

We define

$$
\Xi_{n}\left(k \alpha_{0},-\frac{z}{2}\right)=\int J_{n}^{2}\left(\mathbf{k} \cdot \boldsymbol{\alpha}_{0},-\frac{z}{2}\right) d \Omega
$$

This integral can be solved analytically by expanding the Bessel function in terms of spherical harmonics

$$
J_{n}\left(\mathbf{k} \cdot \boldsymbol{\alpha}_{0},-\frac{z}{2}\right)=\sum_{l m} F_{l m}^{n}\left(k \alpha_{0},-\frac{z}{2}\right) Y_{l m}(\theta, \phi)
$$

where

$$
F_{l m}^{n}\left(k \alpha_{0},-\frac{z}{2}\right)=\int J_{n}\left(\mathbf{k} \cdot \boldsymbol{\alpha}_{0},-\frac{z}{2}\right) Y_{l m}^{*}(\theta, \phi) d \Omega
$$

Using the definition of the Generalized Bessel function, we find

$$
F_{l m}^{n}\left(k \alpha_{0},-\frac{z}{2}\right)=\sum_{k=-\infty}^{\infty} \int J_{n-2 k}\left(\mathbf{k} \cdot \boldsymbol{\alpha}_{0}\right) Y_{l m}^{*}(\theta, \phi) d \Omega J_{k}\left(-\frac{z}{2}\right)
$$

and

$$
F_{l m}^{n}\left(k \alpha_{0},-\frac{z}{2}\right)=\sum_{k=-\infty}^{\infty} \mathbb{J}_{l m}^{n-2 k}\left(k \alpha_{0}\right) J_{k}\left(-\frac{z}{2}\right),
$$

where we have defined

$$
\mathbb{J}_{l m}^{n-2 k}\left(k \alpha_{0}\right)=\int J_{n-2 k}\left(\mathbf{k} \cdot \boldsymbol{\alpha}_{0}\right) Y_{l m}^{*}(\theta, \phi) d \Omega
$$


APPENDIX D. DERIVATION OF THE FUNCTION $\Xi_{N}\left(K \alpha_{0},-\frac{Z}{2}\right)$

Using the definition of the spherical harmonics

$$
Y_{l m}^{*}(\theta, \phi)=(-1)^{l} \sqrt{\frac{2 l+1}{4 \pi} \frac{(l-m) !}{(l+m) !}} P_{l}(\cos \theta) e^{-i m \phi},
$$

we get

$$
\mathbb{J}_{l m}^{n-2 k}\left(k \alpha_{0}\right)=(-1)^{l} \sqrt{\frac{2 l+1}{4 \pi} \frac{(l-m) !}{(l+m) !}} \int J_{n-2 k}\left(k \alpha_{0} \cos \theta\right) P_{l}(\cos \theta) d(\cos \theta) \int e^{-i m \phi} d \phi
$$

or

$$
\mathbb{J}_{l m}^{n-2 k}\left(k \alpha_{0}\right)=(-1)^{l} \sqrt{\frac{2 l+1}{4 \pi} \frac{(l-m) !}{(l+m) !}} \mathcal{J}_{l}^{n-2 k}\left(k \alpha_{0}\right) \delta_{m 0},
$$

where we again define a new function

$$
\mathcal{J}_{l}^{n-2 k}\left(k \alpha_{0}\right)=2 \pi \int J_{n-2 k}\left(k \alpha_{0} \cos \theta\right) P_{l}(\cos \theta) d(\cos \theta) .
$$

Let $x=\cos \theta$, then

$$
\mathcal{J}_{l}^{n-2 k}\left(k \alpha_{0}\right)=2 \pi \int_{-1}^{1} J_{n-2 k}\left(k \alpha_{0} x\right) P_{l}(x) d x
$$

Using this integral expansion of the Bessel function

$$
J_{n-2 k}\left(k \alpha_{0} x\right)=\frac{i^{-(n-2 k)}}{\pi} \int_{0}^{\pi} e^{i k \alpha_{0} x \cos \theta} \cos ((n-2 k) \theta) d \theta
$$

and we find

$$
\mathcal{J}_{l}^{n-2 k}\left(k \alpha_{0}\right)=2 \pi \int_{-1}^{1}\left[\frac{i^{-(n-2 k)}}{\pi} \int_{0}^{\pi} e^{i k \alpha_{0} x \cos \theta} \cos ((n-2 k) \theta) d \theta\right] P_{l}(x) d x .
$$


APPENDIX D. DERIVATION OF THE FUNCTION $\Xi_{N}\left(K \alpha_{0},-\frac{Z}{2}\right)$

By switching the order of integration,

$$
\mathcal{J}_{l}^{n-2 k}\left(k \alpha_{0}\right)=2 i^{-(n-2 k)} \int_{0}^{\pi}\left[\int_{-1}^{1} e^{i k \alpha_{0} \cos \theta x} P_{l}(x) d x\right] \cos ((n-2 k) \theta) d \theta
$$

we can use

$$
e^{i k \alpha_{0} \cos \theta x}=\sum_{l}(2 l+1) i^{l} j_{l}\left(k \alpha_{0} \cos \theta\right) P_{l}(x) .
$$

Then

$$
\begin{gathered}
\mathcal{J}_{l}^{n-2 k}\left(k \alpha_{0}\right)= \\
2 i^{-(n-2 k)} \int_{0}^{\pi}\left[\int_{-1}^{1}\left(\sum_{l^{\prime}}\left(2 l^{\prime}+1\right) i^{l^{\prime}} j_{l^{\prime}}\left(k \alpha_{0} \cos \theta\right) P_{l^{\prime}}(x)\right) P_{l}(x) d x\right] \cos ((n-2 k) \theta) d \theta \\
=2 i^{-(n-2 k)} \int_{0}^{\pi}\left[\sum_{l^{\prime}}\left(2 l^{\prime}+1\right) i^{l^{\prime}} j_{l^{\prime}}\left(k \alpha_{0} \cos \theta\right) \int_{-1}^{1} P_{l^{\prime}}(x) P_{l}(x) d x\right] \cos ((n-2 k) \theta) d \theta \\
=2 i^{-(n-2 k)} \int_{0}^{\pi}\left[\sum_{l^{\prime}}\left(2 l^{\prime}+1\right) i^{l^{\prime}} j_{l^{\prime}}\left(k \alpha_{0} \cos \theta\right) \frac{2}{2 l+1} \delta_{l^{\prime} l}\right] \cos ((n-2 k) \theta) d \theta \\
=4 i^{l-(n-2 k)} \int_{0}^{\pi} j_{l}\left(k \alpha_{0} \cos \theta\right) \cos ((n-2 k) \theta) d \theta
\end{gathered}
$$

Using the series expansion

$$
j_{l}(x)=2^{l} x^{l} \sum_{m=0}^{\infty}(-1)^{m} \frac{(m+l) !}{m !(2 m+2 l+1) !} x^{2 m}
$$

We find

$$
\begin{gathered}
\mathcal{J}_{l}^{n-2 k}\left(k \alpha_{0}\right)= \\
4 i^{l-(n-2 k)} \int_{0}^{\pi} 2^{l}\left(k \alpha_{0} \cos \theta\right)^{l} \sum_{m=0}^{\infty}(-1)^{m} \frac{(m+l) !}{m !(2 m+2 l+1) !}\left(k \alpha_{0} \cos \theta\right)^{2 m} \cos ((n-2 k) \theta) d \theta \\
=42^{l} i^{l-(n-2 k)} \sum_{m=0}^{\infty}(-1)^{m} \frac{(m+l) !}{m !(2 m+2 l+1) !}\left(k \alpha_{0}\right)^{2 m+l} \int_{0}^{\pi} \cos ^{2 m+l} \theta \cos ((n-2 k) \theta) d \theta .
\end{gathered}
$$


APPENDIX D. DERIVATION OF THE FUNCTION $\Xi_{N}\left(K \alpha_{0},-\frac{Z}{2}\right)$

we can simplify this using,

$$
\int_{0}^{\pi} \cos ^{\mu} \theta \cos (\nu \theta) d \theta=\left(1+(-1)^{\mu+\nu}\right) \frac{\pi}{2} \frac{1}{2^{\mu}}\left(\begin{array}{c}
\mu \\
\frac{\mu-\nu}{2}
\end{array}\right)
$$

where the brackets are the combination symbol. We get

$$
\begin{aligned}
\mathcal{J}_{l}^{n-2 k}\left(k \alpha_{0}\right)= & 4 \frac{\pi}{2} i^{l-(n-2 k)} \sum_{m=0}^{\infty}(-1)^{m}\left(1+(-1)^{2 m+l+(n-2 k)}\right) \\
& \left.\times \frac{(m+l) !}{m !(2 m+2 l+1) !}\right) \frac{1}{2^{2 m}}\left(\begin{array}{c}
2 m+l \\
\frac{2 m+l-(n-2 k)}{2}
\end{array}\right)\left(k \alpha_{0}\right)^{2 m+l},
\end{aligned}
$$

or

$$
\mathcal{J}_{l}^{m}(x)=2 i^{l-m} \pi^{3 / 2}\left(\frac{x}{4}\right)^{l} \sum_{j=0}^{\infty}(-1)^{j} \frac{\left(\frac{x}{4}\right)^{2 j}}{j ! \Gamma(j+l+3 / 2)}\left(\begin{array}{c}
2 j+l \\
\frac{2 j+l+|m|}{2}
\end{array}\right) \quad \bmod (l+m, 2)=0
$$

The last eqaution has been discussed by Kylstra and Joachain (Phys. Rev. A 60, 2255 (1999)) [67]. 
APPENDIX D. DERIVATION OF THE FUNCTION $\Xi_{N}\left(K \alpha_{0},-\frac{Z}{2}\right)$

$\Xi_{n}\left(k \alpha_{0},-\frac{z}{2}\right)=\sum_{l=0}^{\infty}\left|F_{n l}\left(k \alpha_{0},-\frac{z}{2}\right)\right|^{2}$
$F_{n l}\left(k \alpha_{0},-\frac{z}{2}\right)=\sum_{\eta=-\infty}^{\infty} \mathbb{J}_{n-2 \eta, l}\left(k \alpha_{0}\right) J_{\eta}\left(-\frac{z}{2}\right)$

